University of Louisville

ThinkIR: The University of Louisville's Institutional Repository

Electronic Theses and Dissertations

8-2014

\title{
Craniodental anatomy of a new late cretaceous multituberculate mammal from Udan Sayr, Mongolia.
}

Amir Subhash Sheth

University of Louisville

Follow this and additional works at: https://ir.library.louisville.edu/etd

Part of the Anatomy Commons, and the Medical Neurobiology Commons

\section{Recommended Citation}

Sheth, Amir Subhash, "Craniodental anatomy of a new late cretaceous multituberculate mammal from Udan Sayr, Mongolia." (2014). Electronic Theses and Dissertations. Paper 1317.

https://doi.org/10.18297/etd/1317

This Master's Thesis is brought to you for free and open access by ThinkIR: The University of Louisville's Institutional Repository. It has been accepted for inclusion in Electronic Theses and Dissertations by an authorized administrator of ThinkIR: The University of Louisville's Institutional Repository. This title appears here courtesy of the author, who has retained all other copyrights. For more information, please contact thinkir@louisville.edu. 
CRANIODENTAL ANATOMY OF A NEW LATE CRETACEOUS MULTITUBERCULATE MAMMAL FROM UDAN SAYR, MONGOLIA

\author{
By \\ Amir Subhash Sheth \\ B.A., Centre College, 2010 \\ A Thesis \\ Submitted to the Faculty of the \\ School of Medicine of the University of Louisville \\ in Partial Fulfillment of the Requirements \\ for the Degree of \\ Master of Science \\ Department of Anatomical Sciences and Neurobiology \\ University of Louisville \\ Louisville, Kentucky
}

August 2014 



\title{
CRANIODENTAL ANATOMY OF A NEW LATE CRETACEOUS
} MULTITUBERCULATE MAMMAL FROM UDAN SAYR, MONGOLIA

\author{
By \\ Amir Subhash Sheth \\ B.A., Centre College, 2010
}

A Thesis Approved on

July $18^{\text {th }}, 2014$

By the Following Thesis Committee:

(Guillermo W. Rougier, Ph.D.)

Thesis Director

(Brian M. Davis, Ph.D.)

(Fabian Crespo, Ph.D.) 


\section{ACKNOWLEDGMENTS}

Thanks to my sisters, Heend and Seema, parents Sawsan and Subhash, and friends and family for enduring my rants about classes, teeth, fossils, and the experience l've had here at UofL. Thanks to my girlfriend, Veronica, for all of her caring support. Thanks to the entire ASNB faculty for their instruction and, occasionally, laughing at my jokes. Thanks to the American Museum of Natural History in New York for access to the Mongolian Multituberculate specimens. Cheers to Brian Davis, the photoshop / illustrator guru, for his guidance and advice. Thanks to Guillermo Rougier who was an excellent, knowledgable, and patient mentor who believed in me, pushed me, and somehow unearthed a scientist from a musician. 


\begin{abstract}
CRANIODENTAL ANATOMY OF A NEW LATE CRETACEOUS MULTITUBERCULATE MAMMAL FROM UDAN SAYR, MONGOLIA

Amir S. Sheth

July 18,2014

Multituberculates were the longest-lived group of early mammals, with a nearly worldwide distribution, and a temporal range from the Middle Jurassic to the Late Eocene. Multituberculates are typically represented by isolated teeth and jaw fragments; however, several localities in Mongolia revealed abundant, well-preserved multituberculate skulls and partial skeletons from the Late
\end{abstract} Cretaceous.

This study is centered on two specimens of a new multituberculate taxon from a locality in the Gobi desert, Udan Sayr. Included is a (1) bone-by-bone description of the cranial and mandibular elements, as well as the dental features of both specimens, PSS-MAE 141 (holotype) and PSS-MAE 142, followed by a (2) phylogenetic analysis, culminating in a (3) discussion regarding comparisons with other multituberculates and the unique middle ear region in the new multituberculate.

The new taxon is a derived member of a specious group of Late Cretaceous Mongolian multituberculates (LCMM), clustering together with largesize forms such as Catopsbaatar, Tombaatar, and Djadochtatherium, forming a monophyletic group. Tombaatar sabuli, also from the Late Cretaceous of 
Mongolia, is the sister taxon and shares with the new form the dental formula, overall dental morphology, and approximate size. The new multituberculate from Udan Sayr has a very large middle ear cavity, housing a petrosal and promontorium that are deeply-sunk into the braincase. The expansion of the middle ear cavity seems to be absent among basal LCMM, only developing among members of Djadochtatherioidea, and to an extreme degree in the Udan Sayr multituberculate. Among living mammals, enlarged middle ear cavities confer enhanced low frequency audition and are often found in fossorial species, such as golden moles, and several groups of rodents. Therefore, a burrowing habit is likely for the new mammal and its closest relatives. The new taxon further demonstrates the diversity of morphologies in multituberculates. 


\section{TABLE OF CONTENTS}

PAGE

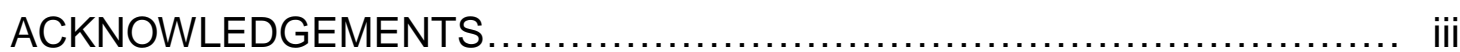

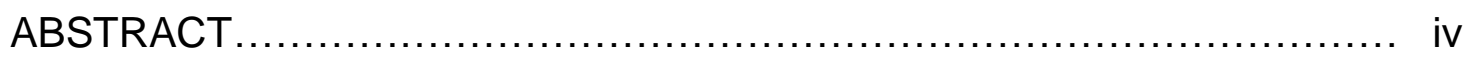

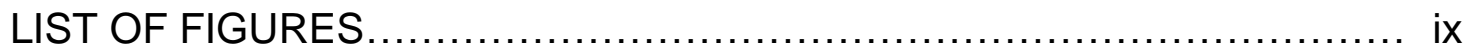

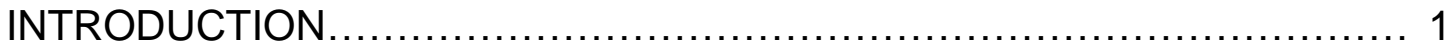

MATERIALS AND METHODS................................................ 6

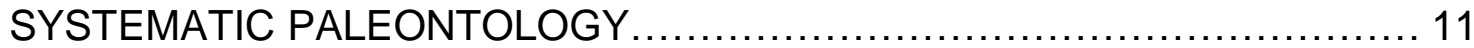

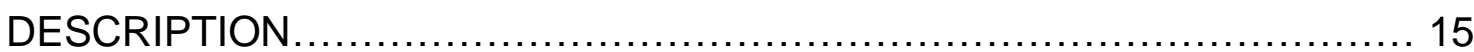

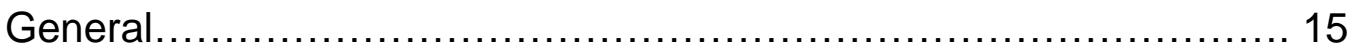

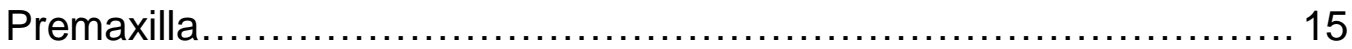

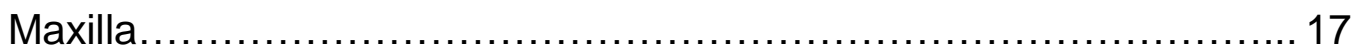

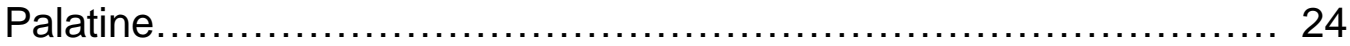

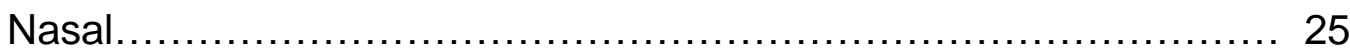

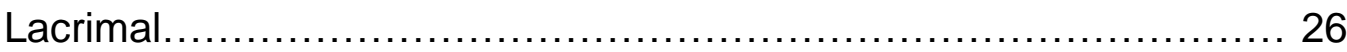

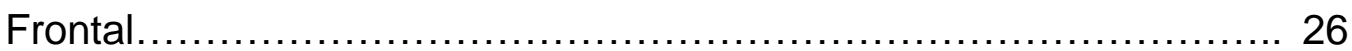

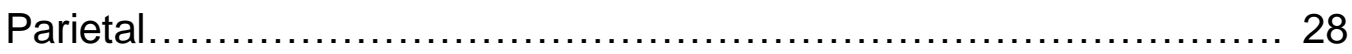

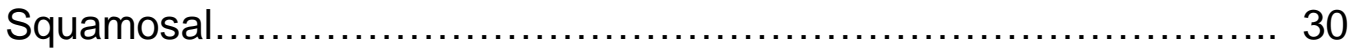

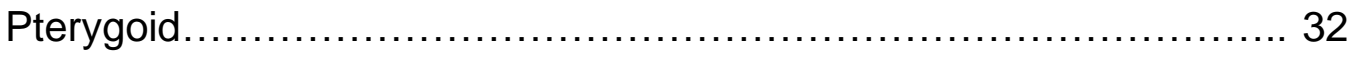




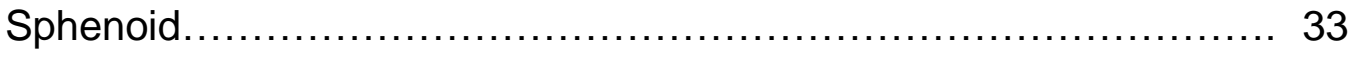

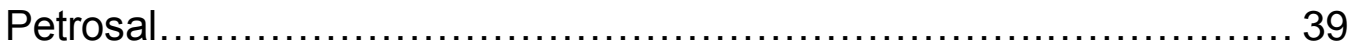

Basioccipital..................................................... 55

Exoccipital............................................................ 56

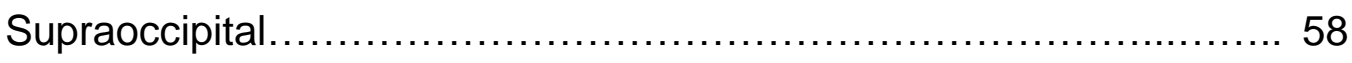

Mandible................................................................ 59

Dentition............................................................ 61

Upper Dentition.................................................... 62

Lower Dentition.................................................... 67

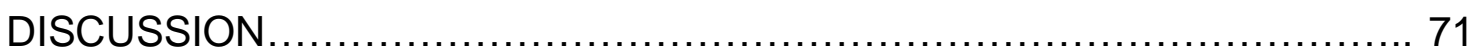

Cladistic Analysis................................................... 71

Discussion of Characters............................................ 74

Status of Djadochtatheriidae, Kielan-Jaworowska, 1997.............. 76

Comparisons with Late Crecateous Mongolian Multituberculates....... 77

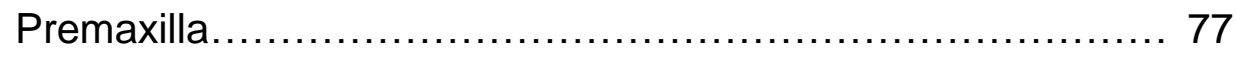

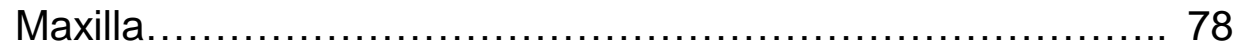

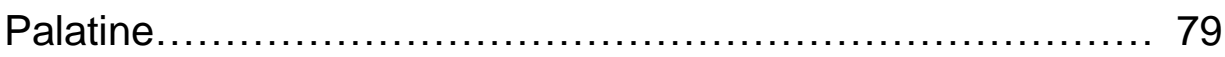

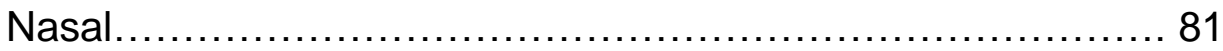

Lacrimal........................................................ 81

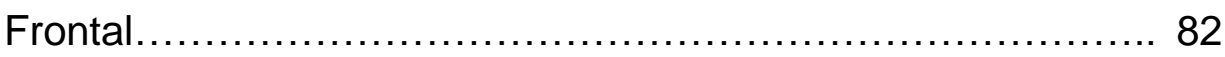

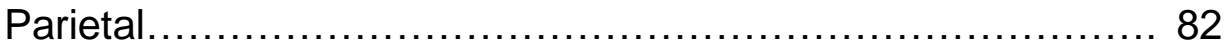

Squamosal...................................................... 83

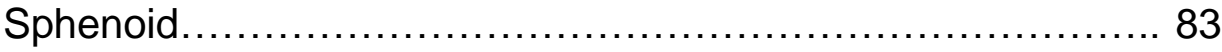


Petrosal......................................................... 84

Middle Ear (Petrosal, Basioccipital, Exoccipital)................ 86

Dentition.................................................... 92

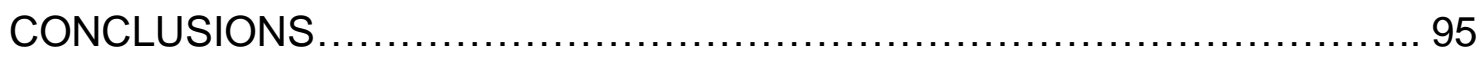

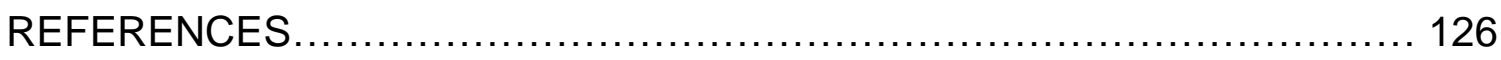

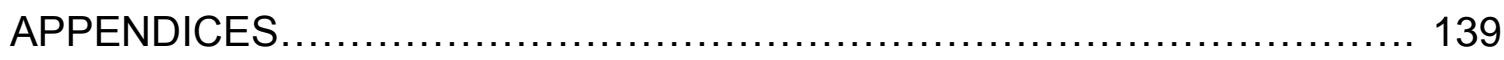

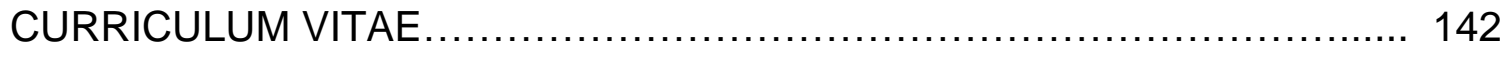




\section{LIST OF FIGURES}

PAGE

Table 1: Full list of anatomical abbreviations................................14

Figure 1: Map of Udan Sayr in the Gobi Desert of Mongolia................... 97

Figure 2: Photograph of Udan Sayr in the Gobi Desert of Mongolia.............. 98

Figure 3: Skull of PSS-MAE 141 in dorsal view with illustration................ 99

Figure 4: Skull of PSS-MAE 141 in ventral view with illustration ............... 100

Figure 5: Palate of PSS-MAE 141 in ventral view........................ 101

Figure 6: Palate (right and left) of PSS-MAE 141 in ventral view............ 102

Figure 7: Illustration of left upper dentition of PSS-MAE 141, occlusal view.....103

Figure 8: Left side of skull of PSS-MAE 141 in lateral view ....................104

Figure 9: Right ear region of PSS-MAE 141 in lateral view ...................104

Figure 10: Basicranium of PSS-MAE 141 in ventral view with illustration........105

Figure 11: Line drawing of basicranium of PSS-MAE 141 in ventral view ..... 106

Figure 12: Left Jaw of PSS-MAE 141 in buccal and mesial views............... 107 
Figure 13: Illustration of the dentary of PSS-MAE 141 in mesial view 108

Figure 14: Dentary of PSS-MAE 141, occlusal view 109

Figure 15: Illustrations of the lower dentition of PSS-MAE 141, occlusal and lateral views 110

Figure 16: Skull of PSS-MAE 142 in dorsal view with illustration 111

Figure 17: Skull of PSS-MAE 142 in ventral view 112

Figure 18: Palate of PSS-MAE 142 in ventral view 113

Figure 19: Skull of PSS-MAE 142 in lateral view (left and right). 114

Figure 20: Reconstruction of the skull of Mangasbaatar in dorsal view 115

Figure 21: Reconstruction of the skull of Mangasbaatar in ventral view. 116

Figure 22: Reconstruction of the middle ear of Mangasbaatar in ventral view. 117 Figure 23: Reconstruction of the skull of Mangasbaatar in lateral view. 118

Figure 24: Reconstruction of the dentary of Mangasbaatar in lateral view......119

Figure 25: Middle ear region (right) of PSS-MAE 142 in ventral view. 120

Figure 26: Middle ear region (left) of PSS-MAE 142 in ventral view. 120

Figure 27: Cladogram showing interrelationships of multituberculates 121

Figure 28: Comparative figure of multituberculate skull reconstructions in dorsal view 122 
Figure 29: Comparative figure of multituberculate skull reconstructions in ventral view. 123

Figure 30: Comparative figure of multituberculate jaw reconstructions in lateral view...... 124

Figure 31: Skull of Kryptobaatar, unpublished specimen, field number PSS-MAE 93-191 in ventral view. 125 


\section{INTRODUCTION}

Since 1990, the American Museum of Natural History (AMNH) and the Mongolian Academy of Sciences have been prospecting the Mesozoic outcrops of Mongolia in search of fossil vertebrates. This new project is the heir of a similar series of expeditions organized by the AMNH during the 1920 s that recovered some of the first early mammals from Central Asia (Gregory and Simpson, 1926; Simpson, 1928a, b; Matthew et al., 1928, 1929). The recent American-Mongolian expeditions (1990 to the present) have followed that tradition and results have been plentiful, including the discovery of one of the richest Mesozoic localities in the world, Ukhaa Tolgod (Norell et al., 1994, 2000, 2001, 2006; Novacek et al., 1994; Dashzeveg et al., 1995, 2005; Rougier et al., 1996a, 1997, 1998; Gao and Hou, 1996; Chiappe et al., 1998; Gao and Norell, 1998, 2000; Makovicky and Norell, 1998; Horovitz, 2000; Wible and Rougier, 2000; Clarke et al., 2001; Clarke and Norell, 2002; Makovicky et al., 2003; Ksepka and Norell, 2004; Norell and Hwang, 2004; Pol and Norell, 2004; Andres and Norell, 2005; Joyce and Norell, 2005; Dingus et al., 2008; Minjin, 2008). The Cretaceous localities of Mongolia, especially those of the Djadochta and similarly aged Barun Goyot Formations (Shuvalov, 2000; Jerzykiewicz, 2000), are exceptionally rich in well-preserved small vertebrates when compared to those in other parts of the world. The joint efforts of the Polish-Mongolian and Russian- 
Mongolian expeditions of the1960s and 1970s resulted in the discovery of new Cretaceous localities in the Nemegt Valley which raised the bar regarding specimen quality and allowed for much more detailed anatomical work than previously possible (Clemens et al., 1979). With very few exceptions, the most complete Late Cretaceous mammalian skeletons known to date have been recovered from those localities (Kielan-Jaworowska, 1969a, b, 1970, 1974, 1975a, b, c, 1977, 1978, 1979, 1984a, b, 2013; Kielan-Jaworowska and Sochava, 1969; Kielan-Jaworowska and Dashzeveg, 1978; Kielan-Jaworowska and Trofimov, 1980; Kielan-Jaworowska and Gambaryan, 1994; Gambaryan and Kielan-Jaworowska, 1995; Kielan-Jaworowska and Hurum, 1997, 2001; KielanJaworowska et al., 2005). Most of the mammalian specimens collected in these Cretaceous localities of Mongolia are multituberculates, a group of mammals which superficially resemble rodents, named for their multi-cusped molars. The Late Cretaceous Mongolian Multituberculates (LCMM) are the best-known specimens of multituberculates and currently eleven genera are recognized (Wible and Rougier, 2000; Kielan-Jaworowska and Hurum, 2001, 2005; KielanJaworowska et al., 2004). To those I add a new form here.

Multituberculates possess the longest fossil record of all mammals, extending from the early middle Jurassic to the late Eocene (Prothero and Swisher, 1992; Freeman, 1976, 1979; Kielan-Jaworowska et al., 2004). Several recent studies (Zheng et al., 2013; Zhou et al., 2013) have opened discussion regarding the timing of certain events within the basal branches of the mammalian tree, namely the origin of the very first mammals via the relationships 
between Haramiyidae and Allotheria. Zheng et al. (2013) describe the preserved mandibles of Arboroharamiya, found in the Tiaojishan Formation of China, with an age estimate from the Middle to Late Jurassic. They conclude that the jaw of Arboroharamiya lacks a postdentary trough, suggesting that the postdentary bones had been reduced to the middle ear ossicles which are considered diagnostic for mammals, ultimately leading them to ally Haramiyidae with Allotheria, making the estimated timing of the appearance of mammals within the Late Triassic. If their interpretation of the postdentary trough is correct then it follows that the mammalian middle ear may have evolved convergently three times, within allotherians, monotremes, and therians. Though the specimen of Arboroharamiya described therein is very well-preserved, the postdentary region is not complete and their interpretation is thus uncertain to some degree. Competing viewpoints were put forward by Zhou et al. (2013) in their recent analysis of Megaconus, found in the same formation but of slightly older age. Megaconus, a more complete specimen within Haramiyidae, consists of a better preserved jaw which clearly displays the inclusion of the postdentary bones within the jaw, thus weakening the argument for the alliance of haramayidans with Allotheria and in turn of haramayidans within Crown mammals. Multituberculates are also one of the best-represented Mesozoic mammals: over five hundred partial or complete skulls and skeletons have been collected from Ukhaa Tolgod between 1993 and 2000. However, the taxonomic diversity in Ukhaa Tolgod and other localities of the Djadochta Formation is relatively low, the fauna being dominated by medium sized forms such as Kryptobaatar 
dashzevegi (Wible and Rougier, 2000). The specimens described here are from a different locality, Udan Sayr (Figures 1, 2), which is supposedly somewhat younger than traditional localities like the Flaming Cliffs, probably corresponding to the Barun Goyot Formation (Jerzykiewicz and Russell, 1991; KeilanJaworowska et al., 2004) and of late Campanian age. A few tantalizing mammalian specimens have been collected at Udan Sayr, including basal metatherians (Trofimov and Szalay, 1994; Szalay and Trofimov 1996), eutherians, and multituberculates (Minjin, 2008), the last of which is reported here.

The collection of complete or nearly complete specimens of LCMM led to a revival in the study of multituberculate phylogeny (Simmons, 1993; Rougier et al., 1997; Kielan-Jaworowska and Hurum, 1997, 2001; Kielan-Jaworowska et al., $2002,2005)$, which has subsided in recent years. One of the most notable results of the work on the late 1990s and early 2000's has been the recognition of an endemic LCMM clade, Djadochtatheria, later reclassified as Superfamily Djadochtatherioidea (Rougier et al., 1997; Kielan-Jaworowska and Hurum, 1997; Kielan-Jaworowska and Hurum, 2001). Multituberculate cranial anatomy has been monographically treated in two instances: Miao (1988) described the taeniolabidoid Lambdopsalis bulla and Wible and Rougier (2000) described Kryptobaatar dashzevegi; later Kielan-Jaworowska and Hurum (2005) provided a revised description of Catopsbaatar, with a good level of detail. Kryptobaatar is a member of Djadochtatherioidea interpreted to occupy a relatively basal position in the group and is a suitable generalized LCMM to serve as interpretative 
baseline for understanding the evolution of Djadochtatherioidea. On the other hand, Tombaatar sabuli (Rougier et al., 1997) is thought to be a much derived member of this clade, but the fragmentary skull so far described is quite poorly preserved. The well-preserved specimens described here are closely related to Tombaatar and provide a substantial amount of new information (of the ear region and basicranium in particular) about this highly derived group. A detailed knowledge of a generalized form, such as Kryptobaatar, and derived forms, such as Tombaatar and allies, will provide a range of morphological diversity for Djadochtatherioidea that will facilitate the interpretation of similar morphologies of multituberculates and other mammals. 


\section{MATERIALS AND METHODS}

Described here are two specimens of a new multituberculate discovered in the Late Cretaceous Mongolian locality of Udan Sayr (44 $03^{\prime} 09^{\prime \prime} N, 102^{\circ} 55^{\prime} 50^{\prime \prime}$ E; See Figures 1, 2). Udan Sayr is, in fact, a series of very small outcrops exposed in a relatively large area. The specimens described here are from the larger of two outcrops within close proximity to each other. During the first visit to this locality in 1997, the group discovered evidence of excavation of large tetrapods. The consensus was that this was also the locality where Udanoceratops (Kurzanov, 1992; Dong and Currie, 1993) was collected. Udan Sayr is also the type locality for Asiatherium reshetovi (Trofimov and Szalay, 1996), a basal metatherian (Rougier et al., 1998), but the exact location where the fossil was collected is unkown. The lithology of Udan Sayr is similar to that of the Djadochta and Barun Goyot formations (Gradziñski et al., 1977; Jerzykiewicz and Russell, 1991; Jerzykiewicz et al., 1993; Loope et al., 1998; Dashzeveg et al., 2005), and the environment is thought to be similarly arid and the age Campanian, likely late Campanian.

The specimens were collected in friable red sandstones that vary from fine to medium in grain size. As in the Djadochta and Barun Goyot formations, the fossils are frequently complete including, in some instances, articulated specimens of which Asiatherium (Trofimov and Szalay, 1996) is a good example. 
Isolated skulls are frequently included in harder carbonate concretions which often result from the erosion of a partial skeleton. The postcranium associated with the specimen PSS-MAE 141 formed the core of a Ph.d. thesis and has been described elsewhere (Minjin, 2008), while PSS-MAE 142 shows portions of the skeleton enclosed in a concretion.

The skull morphology of multituberculates is distinct from that of other mammals and different authors have used various organizational schemes to describe them with a rather elaborated, sometimes incompatible nomenclature (Gidley, 1909; Simpson, 1937; Kielan-Jaworowska, 1971; Kielan-Jaworowska et al., 1986, 2002, 2005; Gambaryan and Kielan-Jaworowska, 1995; Wible and Rougier, 2000; Kielan-Jaworowska and Hurum, 2005). Here, I will follow the nomenclature of Wible and Rougier (2000) as developed in their glossary, incorporating recent additions by Kielan-Jaworowska et al. (2005). The term "Lateral Flange" has been used to denote two slightly different portions of the multituberculate middle ear. Kielan-Jaworowska et al. (2005) followed earlier usage by Kielan-Jaworowska (1971), against that used by Rougier, Wible and Hopson (Rougier et al., 1992, 1996a; Wible and Hopson, 1993; Wible and Rougier, 2000; Rougier and Wible, 2006). Kielan-Jaworowska et al. (2005) reported that Wible and Rougier (2000: 40,94) endorsed the interpretations of Kielan-Jaworowska, (1971) of "Lateral Flange"; that is not so. The referred pages, in particular Wible and Rougier (2000:40), are an almost textual use of Hopson's personal communication (Kielan-Jaworowska et al., 2005:488-489) defining his interpretation of "Lateral Flange". Here, "Lateral Flange" refers to the 
thickened lower edge of the anterior lamina that, in multituberculates, is inturned (medially directed) and contacts the promontorium.

I will present in this contribution a detailed description of the craniodental anatomy of a new multituberculate from the Late Cretaceous Mongolia and will limit comparisons to those forms also occurring in the Mongolian Late Cretaceous. The preliminary comparison and evaluation of the morphological features of these new specimens strongly suggest that they are members of Djadochtatherioidea, an endemic Late Cretaceous multituberculate clade of Asia (Rougier et al., 1997; Kielan-Jaworowska and Hurum, 1997; Kielan-Jaworowska et al., 2003), representing fairly derived members of that clade (see analysis below). In fact, these new specimens are similar to Catopsbaatar catopsaloides from the classical Late Cretaceous locality of Kheermin Tsav (KielanJaworowska, 1994, Kielan-Jaworowska et al., 2002, 2005) and to the type of Tombaatar sabuli (Rougier et al., 1997), a species from the Mongolian Late Cretaceous locality of Ukhaa Tolgod (Dashzeveg et al., 1996; Novacek et al., 1997). Tombaatar was identified, by Rougier et al. (1997) and KielanJaworowska and Hurum (1997), as closely related to another large LCMM, Catopsbaatar catopsaloides (Kielan-Jaworowska, 1974, 1994). Tombaatar, Catopsbaatar, and a third form, Djadochtatherium (Simpson, 1925), are diagnosed by a relatively large suite of derived characters (Rougier et al., 1997; Kielan Jaworowska and Hurum, 1997; Kielan-Jaworowska et al., 2002, 2005) that are also present in this new species. Therefore, following Rougier et al. (1997), and Kielan-Jaworowska and Hurum $(1997,2001)$, this study accepts the 
existence of a group formed by the large sized LCMM and I will discuss the affinities of these specimens in this context.

The specimen PSS-MAE 141 consists of a skull (Figures 3, 4, 5, 6a, b, 7, 8, 9, 10) lower jaws (Figures 11, 12a, b, 13, 14, 15a, b), and postcranial elements (Minjin, 2008). The skull has been dorsoventrally compressed, resulting in a moderate loss of height and a general oblique deformation of the skull to the left when viewed from the front. Missing are portions of the right zygomatic arch and basicranium. Only remnants of the skull roof elements are preserved. The sutures, however, can be followed in most instances. The left lower jaw is nearly complete with full dentition, while the dentary and three teeth are all that remain of the right lower jaw.

A second specimen PSS-MAE 142, attributed to the hypodigm, is represented by an incomplete skull (Figures 16, 17, 18, 19a, b) and postcranial skeleton. The specimen includes a good portion of the rostrum, palate, braincase, fragments of both glenoids, and an isolated right premaxilla. The lower jaws are only partially preserved. The right lower jaw is a fragment showing p3-m2 while on the left only the $\mathrm{m} 2$ has been recovered. The postcranium includes most dorsal lumbar vertebrae, an articulated segment of the caudal series, fragmentary shoulder girdle and forelimbs, and nearly complete pelvis and hindlimbs.

The body mass of both specimens has been estimated following Wilson et. al, (2012) by measuring $\log ($ body mass $)=3.488^{*} \log ($ skull length $)-3.332$. The body mass is calculated in grams while the skull length is measured in 
millimeters (Mangasbaatar has a skull length of $63.0 \mathrm{~mm}$ and an estimated body mass of 879.0 grams).

\author{
Institutional abbreviations
}

AMNH: American Museum of Natural History, New York, USA

IGGP: Collections of the former Geological Museum of the institute of

Precambrian Geology and Geochronology, USSR Academy of Sciences in

Leningrad, Russia

PIN: Paleontological Institute of the Russian Academy of Sciences, Moscow,

Russia

PSS-MAE: Paleontological and Stratigraphic Section (PSS) of the Geological Institute, Mongolian Academy of Sciences, Ulaan Baatar, Mongolia ZPAL: Institute of Paleobiology of the Polish Academy of Sciences, Warsaw, Poland 


\section{SYSTEMATIC PALEONTOLOGY}

Class MAMMALIA

Subclass ALLOTHERIA

Order MULTITUBERCULATA

Superfamily DJADOCHTATHERIOIDEA, Kielan-Jaworowska and Hurum, (2001)

Family DJADOCHTATHERIIDAE, Kielan Jaworowska and Hurum, (1997)

\section{Mangasbaatar, new genus}

Type and Only Species: Mangasbaatar udanii, new species.

Etymology: Mangas, transliteration from Mongolian: Mangas is a mythological monster of Mongolian folklore; baatar, transliteration from the Mongolian for hero, a suffix commonly used to designate Mongolian multituberculates; udanii, after the locality Udan Sayr where the specimens were found. Udan: probably a corruption of the Mongolian Ulaan, meaning red: red wash, or gulch, which describes the locality well.

Holotype: PSS-MAE 141, an almost complete skull and jaws.

Referred Material: PSS-MAE 142, an incomplete skull and jaws associated with a fairly complete postcranium.

Locality: Udan Sayr, 85 km northwest of Bulgan-Somon, Umuni Gobi Aimak (southern region), Mongolia, from beds of similar lithology and age as the Barun Goyot Formation. 
Diagnosis: Large-sized multituberculate similar to Tombaatar and Catopsbaatar, but differing from other Late Cretaceous Mongolian Multituberculates in lacking the P2. Mangasbaatar shares with Tombaatar the proportions of the M1 and a similar cusp formula for the M1 (one extra cusp in each of the rows for Mangasbaatar), but differs in the slightly larger skull size, smaller size of the $\mathrm{P} 1$, and different $\mathrm{M} 1$ to $\mathrm{M} 2$ length ratio (1.78 Mangasbaatar, 1.46 Tombaatar). The M1 in Mangasbaatar is proportionately $20 \%$ longer than in Tombaatar. Mangasbaatar resembles Catopsbaatar, but differs fromTombaatar and Djadochtatherium, in having broad flat nasals. The frontals and parietals in Mangasbaatar are strongly convex, but less so in Catopsbaatar (the condition is unknown in Djadochtatherium and Tombaatar). The proportionally longer molars in Mangasbaatar determine that the root of the zygomatic arch is approximately at the level of the posterior root of the P4/M1 embrasure. Mangasbaatar resembles Catopsbaatar in this feature, but Tombaatar, and probably Djadochtatherium, share the primitive condition with the root of the zygomatic arch more anteriorly positioned at the level of the anterior root of the P4. All of the large sized LCMM share a posterior position for the orbit, a derived feature absent in more generalized LCMM. The ear region of most LCMM lodges large tympanic sinuses. Mangasbaatar's development of these features is extreme and similar to an as yet undescribed specimen of Tombaatar (MAE 96-31). Catopsbaatar seems to have this sinus complex less developed, while in Djadochtatherium the relevant portion of the skull is not known. Mangasbaatar shares with other LCMM except Tombaatar the premaxilla as the only element 
forming the alveolus for P3. Mangasbaatar shares with Djadochtatherium and Catopsbaatar the presence of a polygonal p4. It also shares with Catopsbaatar, but not with Djadochtatherium, an almost vertical anterior wall of the diastema in front of the p3. In Mangasbaatar the occlusal plane of the lower teeth forms a low angle to the plane of the bottom of the lower jaw similar to that in Catopsbaatar, but different than in Djadochtatherium. The p3 still has a clearly differentiated crown in Mangasbaatar, but it is more "peg-like" in Catopsbaatar. Kryptobaatar, Nemegtbaatar, and Chulsanbaatar retain more primitive conditions for these characters of the lower dentition. 
Table 1

Full List of Anatomical Abbreviations

\begin{tabular}{|c|c|c|c|}
\hline al & anterior lamina & maf & masseteric fossa \\
\hline ali & alisphenoid & man & mandible \\
\hline bo & basioccipital & mapf & major palatine foramen \\
\hline bs & basisphenoid & mcp & medial choanal passage \\
\hline ci & crista interfenestralis & mec & middle ear cavity \\
\hline con & (mandibular) condyle & $\mathrm{mf}$ & mental foramen \\
\hline cor & coronoid process & $\mathrm{mp}$ & masseteric protuberance \\
\hline $\mathrm{cp}$ & crista parotica & $\mathrm{mpf}$ & minor palatine foramen \\
\hline ctpp & caudal tympanic process of petrosal & $m x$ & maxilla \\
\hline ef & ethmoidal foramen & na & nasal \\
\hline er & epitympanic recess & or & orbitosphenoid \\
\hline exoc & exoccipital & otc & orbitotemporal canal \\
\hline fbu & foramen bucinatorium & P3 & third upper premolar \\
\hline $\mathrm{ff}$ & facial foramen & p3 & third lower premolar \\
\hline $\mathrm{fi}$ & fossa incudis & $\mathrm{p} 4$ & fourth lower premolar \\
\hline fica & foramen for internal carotid artery & pa & parietal \\
\hline fma & foramen masticatorium & pal & palatine \\
\hline foi & foramen ovale inferium & pat & postpalatine torus \\
\hline fo & fenestra ovalis & pef & perilymphatic foramen \\
\hline $\mathrm{fr}$ & frontal & pet & petrosal \\
\hline frs & foramen for ramus superior & $\mathrm{pmx}$ & premaxilla \\
\hline frt & foramen for ramus temporalis & pop & postorbital process \\
\hline fs & facial sulcus & $\mathrm{ppp}$ & pterygoparaoccipital proc. \\
\hline $\mathrm{fv}$ & fenestra vestibuli & $\mathrm{pr}$ & promontorium \\
\hline gl & glenoid fossa & prc & prootic canal \\
\hline i & lower incisor & pt & pterygoid \\
\hline $\inf$ & incisive foramen & ptc & posttemporal canal \\
\hline iof & infraorbital foramen & spf & sphenopalatine foramen \\
\hline izr & intermediate zygomatic ridge & sphf & sphenorbital fissure \\
\hline jos & jugular fossa & sq & squamosal \\
\hline jf & jugular foramen & stmf & stylomastoid foramen \\
\hline lac & lacrimal & sup & supraoccipital \\
\hline Icp & lateral choanal passage & $\operatorname{tg}$ & temporal groove \\
\hline If & lateral flange & $\operatorname{tr}$ & temporal ridge \\
\hline $\mathrm{m} 1$ & first lower molar & $v_{3}$ & foramina for $v_{3}$ nerve \\
\hline M2 & second upper molar & vo & mer \\
\hline $\mathrm{m} 2$ & second lower molar & & \\
\hline
\end{tabular}




\section{DESCRIPTION}

\section{General}

Mangasbaatar udanii is one of the largest Late Cretaceous Mongolian multituberculates and, along with Catopsbaatar is among the largest mammalian herbivore of the Mesozoic with a body mass estimate of $0.879 \mathrm{~kg}$ (Wilson et. al, 2012). The two specimens described in this paper are preserved well enough to allow a reconstruction of the skull (Figures 20, 21, 22, 23) and jaws (Figure 24). Presented here is a bone-by-bone description of the skulls and lower jaws of the type specimen, PSS-MAE 141, and the referred specimen, PSS-MAE 142. The postcranial skeleton is described elsewhere (see Minjin, 2008). The type specimen is a young adult with only little wear of the cheek teeth, while the referred specimen is an old adult whose cusps have been worn flat.

\section{Premaxilla}

The premaxilla is a large bone with both horizontal (palatal) and vertical (facial) processes well-developed, forming a substantial portion of the muzzle. The horizontal component forms the anterior portion of the palate and the floor of the nasal cavity. In ventral view of PSS-MAE 141, the point of contact between both premaxillae is obscured by the deformation of this specimen. The suture with the maxilla on the rostrum meanders posteromedially from the raised labial margin (crista premaxillaris of Kielan-Jaworowska et al., 2005). Most of the 
external surface of the right premaxilla in PSS-MAE 141 is missing, exposing the broken I 2 inside the alveolus. Enough is preserved of the premaxilla however to show that, as in Tombaatar, the maxilla extensively overlaps the premaxilla. This feature is also shown by the isolated premaxillae of PSS-MAE 142. The premaxilla lodges the ever-growing 12 that bulges into the nasal cavity.

In ventral view the premaxilla-maxilla suture does not form the caudal rim of the alveolus for 13, as in Tombaatar, but rather runs posterior to this alveolus conforming to the condition present in most members of Djadochtatherioidea. Therefore, the circular alveolus for $I 3$ is fully contained within the premaxilla. Both alveoli, I2 and I3, are anteroposteriorly aligned along a parasagittal plane. Medial to the 13 alveoli are the large, anteroposteriorly oriented incisive foramina which are jointly formed by premaxilla and maxilla. As in Tombaatar, the premaxilla is perforated by several nutrient foramina anterior to the incisive foramina and there are distinct thickenings of the premaxilla between the alveoli for I2 and I3 (Rougier et al., 1997). The crista premaxillaris (Kielan-Jaworowska et al., 2005) is well-developed, but lacks most of the nutrient foramina seen in Catopsbaatar. Only a few are present in PSS-MAE 141 and 142.

The vertical component of the premaxilla forms the lateral wall of the nasal cavity and the lower margin of the external nares. The opening of the nares is oriented directly anteriorly and no indication of a septomaxilla or internarial bar is discernible, as in, for example, the Late Cretaceous Multituberculate Lambdopsalis. In lateral view, contact with the maxilla is along a rostrally convex suture posterior to the alveolus of a large I2. A slender posterior process of the 
premaxilla extends between the maxilla and nasal, ending above P1. The narrow extension of the posterior process is similar to Tombaatar and Catopsbaatar but differs from the blunter process of other members of Djadochtatherioidea.

\section{Maxilla}

The maxilla in multituberculates is a large bone that forms part of the palate, rostrum, and orbital areas. These three portions of the bone define individual processes: the palatal, facial, and orbital, respectively. The maxilla contacts the premaxilla anteriorly, the nasals and lacrimals dorsally, and the palatine posteriorly in the palate. Posteriorly in the orbital-temporal area the maxilla contacts the frontal, the alisphenoid, and possibly the orbitosphenoid. The rostral process of the maxilla is large and is the main bone forming the rostrum. It is convex laterally, reflecting the large size of the maxillary sinuses as seen in multitubereculate serial sections (Kielan-Jaworowska et al., 1986; Hurum, 1994) or CT scans (Kik, 2002). This conspicuous lateral bulging of the maxilla gives multituberculates their distinctly triangular appearing rostrum. The extreme development of this feature is seen in members of Djadochtatherioidea where the rostrum and the side of the zygomatic arches become confluent (Rougier et al., 1997; Kielan-Jaworowska and Hurum, 1997, 2001; KielanJaworowska et al., 2004, 2005). The premaxillary-maxillary suture is wellpreserved only on the left side of PSS-MAE 142. The suture forms a long arcuate line that extends posteriorly both at the ventral and dorsal limits. The dominant feature on the rostral process is the large, depressed infraorbital foramen that opens into the rostrum at the level corresponding to the diastema between P1 
and P3. The infraorbital foramen is depressed dorsoventrally and its exit forms a deep groove that extends anteriorly under the bulging sides of the maxilla. Thus far, with the sole exception of some specimens of Catopsbaatar (KielanJaworowska et al., 2005) all late LCMM have been described as having only one infraorbital foramen. This is a derived condition shared by all cimolodonts but absent in the Jurassic paulchoffatiids (Hahn 1985, 1987; Hahn and Hahn, 1994; Simpson 1928a). In Mangasbaatar, there is a second, small foramen anterior to the main infraorbital foramen just described. This small aperture opens at the level of the P1, or slightly in front of it, and is present bilaterally in PSS-MAE 141 and 142. Given the distribution of the character among the remaining LCMM it is likely that the presence of this secondary infraorbital foramen in Mangasbaatar, and those variably present in Catopsbaatar, are a convergence to the primitive morphology. The condition of this character in the closely related Tombaatar cannot be ascertained in the type specimen because of the poor preservation of the relevant area, but other available specimens show a single foramen.

The infraorbital canal is broken open on both sides of PSS-MAE 142 so that the communication between the maxillary foramen in the orbit and the infraorbital foramen in the rostrum can be traced. The left side of the specimen has been prepared revealing several foramina of various sizes that open medially from the infraorbital canal. These foramina correspond to those described by Rougier et al. (1997) in Tombaatar and Djadochtatherium. These foramina likely correspond to alveolar nerves providing blood and nervous supply to the teeth 
and maxillary sinus. A similar pattern is known among recent mammals (Sisson and Grossman, 1955; Evans and Christensen, 1979; Moore and Agur, 2002).

The external surface of the maxilla in PSS-MAE 142 is well-preserved and shows numerous small nutrient foramina perforating the substance of the bone. A distinctive pattern of small ridges and rugosities mostly oriented anteroposteriorly are present on the lower half of the maxilla indicating the area of attachment of superficial facial musculature. The $m$. buccinatorius is, most likely, responsible for the scars mentioned above.

The rostral process continues posteriorly to form, together with the lacrimal, the anterior edge of the orbit. As in all other LCMM, Mangasbaatar lacks a distinct floor for the orbit, and the maxilla shows a well-developed orbital pocket in front of the orbital ridge (Kielan-Jaworowska et al; 1986; Gambaryan and Kielan-Jaworowska, 1995; Wible and Rougier, 2000). This orbital pocket accounts for approximately one-third of the length of the preorbital region of Mangasbaatar. The root of the zygomatic arch marks the anterior extent of the orbital pocket. The zygomatic process of the maxilla is broad, originates at the level of the embrasure between P4 and M1, and extends backward to contact the zygomatic process of the squamosal at roughly the lowest point of the orbit. The dorsal edge of the zygomatic process is concave-convex determining a very peculiar orbital outline, shared with Tombaatar. The area forming the orbital edge is slightly convex, becoming concave posteriorly, towards the maxillo-squamosal suture; this arrangement results in a very slender posterior portion of the 
zygoma. The great height of the zygomatic arch produces a relatively small, dorsally located orbit, located in the posterior half of the skull.

Between the root of the zygoma, the infraorbital foramen, and P1-P4, there is a flat area that forms a distinct platform dorsal to the teeth. A sharp ridge, the anterior zygomatic ridge, divides this platform. The anterior zygomatic ridge ends directly lateral to the anterior root of P4 and extends posteriorly into the zygoma (although the exact distal extension cannot be determined because of insufficient preservation). Enough is preserved in isolated maxilary fragments of PSS-MAE 142 to show that the ridge is well developed with a lightly rugose apex. What is preserved of the anterior zygomatic ridge conforms to the morphology present in other LCMM, but is longer and narrower than that present in Catopsbaatar (Kielan-Jaworowska and Hurum, 2005).

In ventral view, the palatal process of the maxilla is preserved in both PSS-MAE 141 and 142. Nevertheless, both specimens are broken and parts of the palate have been displaced and asymmetrically deformed. The result of this deformation is that right and left elements are not always aligned. The sutures are better shown by PSS-MAE 141, but PSS-MAE 142 conforms quite closely to the pattern of the type. The maxillary contribution to the incisive foramina is not well preserved in either specimen, though it seems limited to the posterior-most edge of the apertures. The contact of the skull between the premaxilla and maxilla in this region has already been described though, in addition, along the suture on the right side of both PSS-MAE 141 and 142, there is a medium sized foramen in the suture right behind I3. The anterior portions of the palatine 
processes of the premaxillae are moderately concave becoming more so posteriorly. This change in curvature of the maxilla is so pronounced that the palate can be considered almost flat at the level of P1 but very deep at the level of $\mathrm{M} 1$.

The maxilla contacts the subrectangular exposure of the palatine approximately at the level of the middle of M1. The contact between maxilla and palatine is made through an inverted "L"-shaped suture with an anterior transverse component and a posterior parasaggital component. A large foramen can be distinguished at the lateral extent of the transverse suture between the maxilla and the palatine bones. It is the greater palatine foramen that grooves the maxilla only in the immediate vicinity of the opening. The foramen probably transmitted the greater palatine artery and nerve that in modern mammals supply the roof of the mouth, to finally anastomose with the arteries and nerves reaching the incisive foramen from the nasal cavity, likely branches of the sphenopalatine artery and nasopalatine nerve.

The longitudinal portion of the maxillary-palatine suture runs closely to the $\mathrm{M} 2$ and the posterior portion of M1. Along the longitudinal portion of this suture there is a slit-like foramen interpreted here as the lesser palatine foramen that opens in front of the M2. Kielan-Jaworowska et al. (2005), followed earlier interpretations (Kielan-Jaworowska et al., 1986) and identified the slit-like foramina, here identified as lesser palatine foramina (following Wible and Rougier, 2000), as a deep pocket called "palatonasal notch". Examination of CT 
scans of Kryptobaatar skull PSS-MAE 101 reaffirms the interpretation of Wible and Rougier (2000) and is thus followed here for Mangasbaatar.

The maxilla has no contribution to the transverse aspect of the palate along the saggital suture with the palatine. The maxilla is restricted to the bone immediately around the roots of the molars, the alveolar process. Immediately behind the M2 the maxilla contacts the alisphenoid through a sharply serrated suture.

The maxilla is extensively exposed in the orbital-temporal region; its orbital portion is broad and flat with the ascending process forming the medial wall of the orbit and contacting dorsally with the lacrimal. The fronto-maxillary suture is extensive and runs obliquely from the anterodorsal aspect of the orbit to the sphenopalatine foramen that occupies a posteroventral position in the orbit. After reaching the sphenopalatine foramen the fronto-maxillary suture becomes approximately horizontal and runs posteriorly along the floor of the temporal area toward the sphenorbital fissure. In the deeper part of the temporal area the frontal is replaced by the orbitosphenoid in its contact with the maxilla. The maxilla also contacts the alisphenoid in the floor of the temporal region. The suture between these two elements is well-shown by PSS-MAE 142. This serrated contact runs from immediately behind M2 to the lateral edge of the sphenorbital fissure. It follows, from the above-described contacts with the maxilla, that a prong of this bone is wedged between the alisphenoid, frontal, and probably the orbitosphenoid in the floor of the temporal area. This wedge of the maxilla forms a platform, originating in the vicinity of the sphenorbital fissure, 
which opens and broadens anteriorly to become continuous with the orbital contribution of the maxilla.

The sphenopalatine foramen is large, with a "dumb-bell" shape, and incompletely divided in half by a process that is well-preserved only in the right side of PSS-MAE 142. The sphenopalatine foramen transmits the sphenopalatine artery and nerve, and the major palatine nerve and artery. Following recent models (Evans and Christensen, 1979), the former pair will occupy the anterodorsal subdivision of the sphenopalatine foramen on its way to the nasal cavity, while the latter pair would occupy the larger posteroventral subdivision of the sphenopalatine foramen on its way to the palate. Along the maxillo-frontal suture on the floor of the orbitotemporal region there is a broad surface, the sphenopalatine groove, that leads to the sphenopalatine foramen.

As in Kryptobaatar (Wible and Rougier, 2000) and Tombaatar (Rougier et al., 1997) there is a small foramen, posteroventral from the sphenopalatine foramen, which transmits the minor palatine nerve and companion vessels from the orbit into the minor palatine foramen of the hard palate. This foramen is preserved only on the right side of PSS-MAE 141. In the type specimen of Tombaatar the orbital entrance of the minor palatine nerves and vessels and the minor palatine foramina are connected by a groove on the maxilla that is open to the choanae, and therefore leaves no doubts that the "palatonasal notch" transmitted a structure to, or from, the orbit to the palate; the status of this feature is Mangasbaatar cannot be ascertained. 


\section{Palatine}

The palatine is well-preserved in both PSS-MAE 141 and 142. In Mangasbaatar the palatine is exposed only in the palate. There is no orbital exposure of this bone as in most other LCMM, with the possible exception of Nemegtbaatar (Hurum, 1998a). Right and left palatines meet in the saggital plane to form a sharp median crest that preserves an open suture throughout its length. The crest becomes taller posteriorly where it meets the robust postpalatine torus. The palatine is essentially flat between the median crest and its lateral contact with the maxilla.

Bordering the ventral-lateral margin of the choanae there is a massive post-palatine torus. This is an unusually complex structure that resembles that of Tombaatar. The torus begins anteriorly at the level of the M1-M2 embrasure, where it rises abruptly from the palate. The torus is formed by two distinct wings, right and left, which project ventrally to the level of the occlusal plane. The wings are concave posteriorly and are limited medially by two sharp crests that result from the bifurcation of the median crest described above in the anterior portion of the palatine. Between these two crests that form the medial edge of the wings of the torus there is a deep recess occupying the midline. The function of this elaborate torus in Mangasbaatar is uncertain. The posterolateral corner of the torus extends posteriorly on the sides of the choanae through a "splint"-like process directly medial to the alisphenoid. This posterior extension of the palatine into the choanae is preserved only in PSS-MAE 141. 
Nasal

The nasals are large paired bones that form the roof of the nasal cavity. They are in contact with each other medially, with the premaxillae and maxillae laterally, and with the frontals posteriorly. Erosion of the dorsal surface of PSSMAE 141 has destroyed most of the nasals, leaving the crest and sutures that projected into the nasal cavity. The overall features of the nasal are preserved mostly as natural molds. Enough remains, however, to show that the nasals become broader posteriorly and are overlapped by the frontals.

In PSS-MAE 141 (Figure 3), the dorsal surface of the nasal cavity and the paranasal sinuses are exposed because of missing nasals and maxilla. Along the suture between nasal and maxilla there is a ridge of bone that projects ventrally into the nasal cavity. This roughly parasaggital ridge is the remnant of the nasalturbinal ridge. Throughout its length the nasal seems to be mostly cancellous bone with only a thin, dense layer of cortical bone. Based on CT scanning of other Mongolian multituberculates like Kryptobaatar (PSS-MAE 101), it is likely that those intranasal cavities were connected to the nasal cavity, indicating an extreme degree of pneumatization of the multituberculate skull. The great development of pneumaticity is frequently reflected by the preservation of the specimens. When found as isolated specimens, the skulls are often missing the thin cortical bone forming the outer surface of nasals, frontal and parietals.

In the specimen PSS-MAE 142 (Figure 4), the posterior one-third of the right nasal is well-preserved, as is a small portion of the left nasal. Along the midline, right and left nasals are separated by a broad prong of the frontals that 
extends anteriorly at about the level of the lacrimal. This process of the frontal and the concomitant separation of the back part of the nasals is a primitive feature for mammals, present in various degrees almost universally among Mesozoic forms. Catopsbaatar has a more transverse nasal-frontal suture than most other LCMM (Kielan-Jaworowska et al., 2005). Not enough is preserved to determine the number of nasal foramina, but judging from the natural endocast it is likely that at least one relatively large foramen was present.

\section{Lacrimal}

Portions of the lacrimal are preserved in both specimens, the most complete being the right side of PSS-MAE 142 (Figures 4, 14). The external exposure of the lacrimal is subrectangular and is wedged in the orbital margin between frontal and maxilla. The nasal contacts the lacrimal along its anteromedial edge. The lacrimal contribution to the orbital mosaic cannot be fully ascertained, but it is clear that it was restricted to the dorsal portions of the orbit without extensive ventral projection. The lacrimal was, however, involved in the formation of a very deep orbital pocket and sharp orbital ridge for the attachment of the anterior portion of the middle zygomatic muscle. Remnants of a bifid nasolacrimal canal are present on the right side of PSS-MAE 142 and most of its course can be traced through the denuded skull roof of the left side of PSS-MAE 141.

Frontal

Most of the contribution of the frontal to the skull roof is missing in PSSMAE 141 (Figure 3), however, the orbital portions of this bone are well-preserved 
(Figure 13). The right frontal in PSS-MAE 142 is mostly complete (Figures 4, 14). Sutures in the skull roof are well shown by PSS-MAE 142 but those in the orbit are somewhat ambiguous in both specimens, especially in the area near the sphenorbital fissure. The frontal is the largest component of the skull roof and extends roughly from the level of the anterior root of M1 until the posterior onethird of the braincase. This moderately convex bone is slightly raised in the midline where it contacts the frontal from the other side via an open suture. Anteriorly the frontal is wedged between the nasals and anterolaterally contacts the lacrimal. The parietal demarcates the posterior expansion of the frontal. The frontals contact the parietals through a broad "V"-shaped suture extending from just in front of the post-orbital process backwards. The frontal forms the dorsalmost portion of the rugose orbital edge. The lateralmost extension of the dorsal orbital rim is damaged in both specimens; however the right side of PSS-MAE 142 may preserve the some evidence of this feature.

In the orbit, the frontal forms most of the posteromedial wall, contacting the orbitosphenoid throughout much of its length and reaching the dorsal-most aspect of the anterior lamina through a narrow process. The frontalorbitosphenoid suture forms a gentle arch from the floor of the orbitotemporal region to the dorsal portion of the anterior lamina. Approximately in the middle of this arch there is a large foramen formed jointly by frontal and orbitosphenoid, the ethmoid foramen; grooves from above and below the foramen lead to it. The one from below, the larger of the two, starts in the sphenopalatine groove and through a gentle curve reaches the ethmoid foramen from directly below. This 
groove follows the likely course of the frontal-orbitosphenoid suture in the area and it was likely occupied by the ethmoid nerve and artery. The groove reaching the ethmoid foramen from above has a sigmoid shape and is developed mostly above the frontal-orbitosphenoid suture, in the frontal. This groove is continuous with the anterior opening of the orbitotemporal canal that is broken open on both sides of PSS-MAE 141. The rostral end of the orbitotemporal canal is formed mostly by the frontal and is completed by the parietal that forms its roof. The likely occupant of this groove was a large orbitotemporal artery and its accompanying vein. A similar pattern is known in most LCMM including Kryptobaatar (Wible and Rougier, 2000). The anterodorsal portion of the frontal contributes to the orbital ridge and orbital pocket that is jointly formed by maxilla, lacrimal, and frontal.

\section{Parietal}

The parietal has been mostly eroded away in PSS-MAE 141 (Figure 3) but most of it is preserved in the right side of PSS-MAE 142 (Figure 4). The parietal contributes to the posterior one-third of the braincase and helps separate the orbit from the temporal area by forming a post-orbital process. The parietal contacts with the frontal anteriorly, through the already described broad, "V"-like suture anteroventrally with the anterior lamina, posteroventrally with the squamosal, and posteriorly with the supraoccipital. The parietal is slightly convex dorsally. This feature becomes more pronounced laterally than in the midline. Right and left parietals are separated by a suture that is difficult to trace because of partial fusion between these bones. 
Temporal lines are present but are not very sharp and they do not meet each other to form the saggital crest. Instead, they contact the lambdoidal crest parasaggitally. The temporal lines originate from the posterior edge of the postorbital process and extend posteromedially forming a broad arch. The relatively lateral position of the temporal lines implies a proportionally small area for the temporalis muscle, much smaller than that in other forms with a saggital crest such as Kryptobaatar (Wible and Rougier, 2000).

The parietals are the sole elements forming the median portions of the lambdoidal crests (i.e. there is no supraoccipital participation). These crests are minimally developed saggitally but flare out and become quite large laterally. This development of the lambdoidal crests begins on the lateral portions of the parietal contribution to these crests and becomes more pronounced in the squamosal/petrosal portion of the crests.

As in all the other LCMM the post-orbital process is formed in its entirety by the parietal. It is preserved in its full length only on the left side of PSS-MAE 141. The process is inordinately long at $10.2 \mathrm{~mm}$ (measured from the root of the process). A very long process of similar dimensions is present in Catopsbaatar (Kielan-Jaworowska, 1974, 1994), another large LCMM probably related to both Tombaatar, and Mangasbaatar (Rougier et al., 1997; Kielan-Jaworowska and Hurum, 1997).

At the base of the post-orbital process is the opening of the orbitotemporal canal, which has already been described as being formed jointly by the frontal and parietal. The orbitotemporal canal is open to the braincase as shown by the 
endocast of the skull PSS-MAE 141. The parietal forms the roof of the canal but it has no floor (is open to the cranial vault) until it reaches the petrosal in the back portion of the braincase (see below).

\section{Squamosal}

The squamosal has sustained damage in both specimens, but the one on the left side of PSS-MAE 141 is the most complete (Figures $3,12,16)$. The squamosal can be subdivided into two portions: the squama abutting the braincase, and the zygomatic process contacting the maxilla and bearing the glenoid fossa. The squamosal contacts the maxilla anteriorly, the petrosal medially, and the parietal dorsally.

As in all other multituberculates the zygomatic arch is formed mostly or solely by the jugal and maxillary bones. In Mangasbaatar, due to the posterior location of the orbit, the zygomatic arch is very short and throughout its length forms the ventral edge of the relatively small orbitotemporal fossa, characteristic of LCMM. In other LCMM a very small jugal has been described as occupying the medial surface of the arch (Hopson et al., 1989; Wible and Rougier, 2000), but preservation in PSS-MAE 141 and 142 is not sufficient to confirm or deny the presence of this element. The contact of the squamosal with the maxilla occurs through a relatively long and oblique suture seen on the left side of PSS-MAE 141 that reaches anteriorly to the level of the anterior edge of the orbit. The shape of the anterior portion of the zygomatic process is very conspicuous and characteristic of Mangasbaatar and Catopsbaatar (Tombaatar unknown); unlike other LCMM the squamosal achieves its maximum dorsoventral development at 
the posterior end, achieving a greater height than the lowest part of the zygomatic process.

Most of the lateral surface of the very short zygomatic process of the squamosal is occupied by a concave surface limited dorsally by a blunt ridge.

This is the intermediate zygomatic ridge, which might marginally extend into the maxillary's contribution to the zygomatic arch. Behind the intermediate zygomatic ridge, the squamosal becomes narrower and more robust forming a neck connecting the glenoid with the braincase. The glenoid is best seen in ventral view (Figures 6, 16, 17, 18) and is teardrop-shaped with its broader portion oriented posteriorly. There is no post-glenoid ridge, but a broad crest continuous with the lower edge of the zygomatic arch marks the lateral extent of the glenoid. The articular surface is mostly flat, with a shallow anterior concavity.

Behind the glenoid, the squamosal neck forms a flat area that ultimately becomes continuous with the epitympanic recess of the petrosal. The medial extension of the neck reaches the braincase, broadens slightly and becomes the squama of the squamosal. The squama overlies the petrosal and does not contribute directly to the braincase proper. The anterior extension of the squama is limited by its contact with the anterior lamina of the petrosal. The suture between these two elements is not very clear in any of these specimens, but it is best on the left side of PSS-MAE 141. The right squamosal of PSS-MAE 142 (Figure 6) is completely lost, exposing its contact with the underlying petrosal. This specimen shows that the squamosal forms the lateral and dorsal walls of the ascending canal as described in Kryptobaatar and Vincelestes (Rougier et al., 
1992; Wible and Rougier, 2000). The squama of the squamosal also forms part of the ventrolateral portion of the lambdoidal crest overlying the mastoid exposure of the petrosal.

\section{Pterygoid}

The area of the skull presumed to be formed by the pterygoids is preserved only in PSS-MAE 141 (Figure 6) but no sutures are visible in the specimen. This is not to say that sutures were not present, but fractures make recognition of them difficult. In order to describe these elements in Mangasbaatar, particular reference is given to the morphology present in other LCMM. Specifically, the pterygoids are interpreted as forming the two tall, parasaggital flanges present in the choanae following a similar pattern as seen in Kryptobaatar (Wible and Rougier, 2000). There is a suture at the level of the posterior extent of the pterygoids connecting right and left elements. Behind this suture and between the flanges of the pterygoids there is a triangular element on the roof of the choanae interpreted as the basisphenoid. If this interpretation is correct the pterygoids would meet along the midline, in front of the vomer, and they would be separated by the latter along the posterior one-third of their length.

The pterygoid flanges are tall and subdivide the choanae into three passages: a broad median one, between right and left lamina, and two lateral ones, between the lamina and the walls of the choanae. The full anterior extent of the pterygoid lamina cannot be traced into the choanae because the left one is incomplete and the right one is obscured by matrix. The lamina end posteriorly in a short, rounded process separated from the main portion of the lamina by a 
shallow notch. This notch and process are likely to be homologous to the hamular process, serving as a "pulley" for the $m$. tensor velii palatini, a muscle originated from the primitive pterygoid musculature of non-mammalian cynodonts (Barghusen, 1986).

In the lateral troughs, which are between the pterygoid laminae and the walls of the choanae, the pterygoid forms most, or all, of the roof and medial walls of the enormously excavated lateral connection of the nasopharynx and the middle ear; this space serves as an equivalent of the Eustachian Tube of later mammals. The exact contribution of the pterygoids to this region, however cannot be fully determined.

\section{Sphenoid}

The sphenoid is at least partially preserved in both specimens and lacks sutures delimiting its individual components, namely the presphenoid, alisphenoid, orbitosphenoid, and basisphenoid. The individual names will be used here in reference to standard anatomical areas formed by these components in other mammals for which the embryological origin of the individual elements is known or presumed.

The alisphenoid has contributions to the palate, choanae, mesocranial region, and middle ear cavity. The three-dimensional arrangement of the alisphenoid is complex so that this element is exposed in ventral and lateral views, in addition to a sizeable portion around the sphenorbital recess that is not accessible in any of these views. 
The alisphenoid is the portion of the sphenoid forming the lateral wall of the braincase in most mammals. In multituberculates the alisphenoid is small (Kielan-Jaworowska, 1971; Kermack and Kielan-Jaworowska, 1971; KielanJaworowska et al., 1986; Wible and Rougier, 2000), probably reduced from a primitive condition in which it was large (Hopson and Rougier, 1993; Rougier and Wible, 2006). This reduced alisphenoid in multituberculates forms a small portion of the anteroventral area of the lateral wall of the cavum epiptericum (Kermack and Kielan-Jaworowska, 1971). In Mangasbaatar, the alisphenoid, as recognized here, is also small and its braincase contribution is limited to the area surrounding the cavum epiptericum. The alisphenoid, however, has a long anterior process that reaches the back of the palate. The alisphenoid contacts the palatine, maxilla, pterygoid and petrosal, and it becomes confluent with the basisphenoidal and orbitosphenoidal portions of the sphenoid. In ventral view, the most prominent feature of the alisphenoid is its anterior process that extends to the hard palate. A little slip of the alisphenoid forms part of the minor palatine foramen and wedges its way between the maxillary and palatine contributions to the rim of the foramen (Figure 18). The anterior process of the alisphenoid has been described as having a möbius strip shape (Wible and Rougier, 2000), resembling the shape of a twisted ribbon. The alisphenoid of Mangasbaatar presents the same basic shape and, because of this "twisting" of the anterior process, the anterior, ventrally oriented surface becomes laterally oriented towards the back. This anterior portion of the alisphenoid is concave and merges anteriorly into the maxilla. The medial limit of the alisphenoid in this area is 
formed by a ridge running along the edge of the choanae and its posterior limit is a tall and sharp crest that also bounds the middle ear cavity. This concave anterior surface is likely to provide the attachment for the medial pterygoid musculature. The alisphenoid is also likely to contribute to the formation of the lateral choanal passage or trough and contact the pterygoid in doing so. However, preservation in both specimens is deficient in this area and the morphology of the lateral wall of the choanae is, to some degree, uncertain.

Behind (distal to) the already mentioned sharp crest in the alisphenoid is the middle ear contribution of the alisphenoid. The alisphenoid forms approximately one-fourth of the epitympanic recess. The alisphenoid portion of the epitympanic recess is restricted to the anterior pole and forms the very prominent anterior and lateral walls of the recess. A thin, narrow process projects back medial to the petrosal contribution to the epitympanic recess to almost reach the tip of the promontorium. Preservation on PSS-MAE 141 is not very satisfactory in this region; it is clearer to follow the morphology in PSS-MAE 142. In the epitympanic recess there are two large foramina for branches of $V_{3}$. The most anterior of these foramina is very near the serrated alisphenoid-petrosal suture, but the foramen is formed solely by the petrosal.

In lateral view, the alisphenoid is limited posteriorly by the tall ridge that marked the posterior edge of the möbius strip in ventral view. The alisphenoid in this view (lateral) is the continuation of the alisphenoid surface immediately behind the palate in ventral view. The lateral aspect of the alisphenoid is best shown on the left side of PSS-MAE 142 and the right side of PSS-MAE 141. This 
surface is concave dorsally and anteriorly, extending toward the sphenorbital recess medial to the anterior lamina. This surface of the alisphenoid is broader anteriorly and tapers posteriorly. There is a small foramen anteriorly directed in the anterior lamina-alisphenoid suture or in its proximity depending on the specimen. Similar variation has been reported for Kryptobaatar (Wible and Rougier, 2000). The foramen is lodged in a deep recess and, leading anteriorly from it, there is a deep groove. Given the orientation and position of the foramen, it is likely that this structure conveyed the buccal branch of the trigeminal nerve (V) and it is thus identified here as the foramen buccinatorium, which also occurs among other multituberculates (Kielan-Jaworowska et al., 1986; Hurum, 1998b; Wible and Rougier, 2000). Posteromedially from the foramen buccinatorium, the alisphenoid occupies the floor of the sphenoidal recess. Laterally, it contacts the tall petrosal wall that guards the lateral extension of the sphenoidal recess. Sutures between the anterior lamina of the petrosal and the alisphenoid in this area are not apparent.

The orbitosphenoid portion of the sphenoid is a laminar process that forms the medial wall of the orbitotemporal fossa connecting the skull base with the elements forming the skull roof and the rear of the nasal cavity. The orbitosphenoid contacts the frontal dorsally and anteriorly, the maxilla anteriorly, the anterior lamina posteriorly, and is continuous with the rest of the sphenoid posteromedially. The orbitosphenoid has extensive contact with the frontal, and together these bones form the ethmoid foramen in the anterodorsal aspect of the temporal area. The dorsal suture with the frontal is shown in the right side of 
PSS-MAE 141. It is a smooth line running obliquely from the vicinity of the anterior opening of the orbitotemporal canal to the ethmoid foramen. The contact between frontal and orbitosphenoid under the ethmoid foramen, as already mentioned, is less clear. With the exception of the left side of PSS-MAE 141, most of the orbitosphenoid spanning from the sphenoidal recess to the ethmoid foramen is missing in both specimens. The left side of the skull PSS-MAE 141, however, is crushed and the suture pattern cannot be made out.

The orbitosphenoid abuts the medial aspect of the anterior lamina and forms the medial edge of the sphenorbital fissure. In PSS-MAE 142 the skull is deformed so that the fissure and its contents can be clearly seen, through the artificially enlarged fissure. The sphenorbital fissure is a large, oval-shaped foramen leading anteriorly. It transmits the contents of the cavum epiptericum, specifically $V_{2}$ and the ethmoidal artery. Directly medial to the edge of the sphenorbital fissure, and slightly above its midpoint, there is a small foramen piercing the orbitosphenoid. This foramen is interpreted here as transmitting the oculomotor nerve (III). The multituberculates Chulsanbaatar, Nemegtbaatar, Kryptobaatar, and Sloanbaatar, have each been identified as having a separate foramen for CN III (Hurum, 1998b; Rougier et al., 1997; Wible and Rougier, 2000). Medial to the oculomotor foramen, slightly above its level, in the deepest part of the orbit, there is a circular foramen in both specimens PSS-MAE 141 and 142: the optic foramen. A broad, shallow sulcus extends anterodorsally from the optic foramen, grooving the external surface of the orbitosphenoid. The portion of the orbitosphenoid directly in front of and ventral to the optic foramen is the 
jugum sphenoidale (Wible and Rougier, 20000) which, in multituberculates, is relatively narrow and proportionally tall. This is also the condition in Mangasbaatar. Anteroventral to the optic foramen, there is another foramen of relatively large size in both specimens that penetrates the substance of the sphenoid. This corresponds to the transverse canal identified in Kryptobaatar (Wible and Rougier, 2000). The size of the transverse canal in Mangasbaatar is substantial and it grooves, slightly, the floor of the sphenorbital recess.

The orbitosphenoid appears to have been convex externally and would have essentially provided support (ventrolateral) for the frontal lobes of the brain. The orbitosphenoid contacts the anterior lamina immediately under the anterior opening of the orbitotemporal canal. The suture between these two elements is best shown on the right side of PSS-MAE 141. This suture is heavily interdigitated and runs almost vertically down towards the sphenorbital recess, but the ventral portion of the suture is not recognizable.

The presphenoid, if present in Mangasbaatar, is not recognizable in the specimens currently available. What remains of the sphenoid can be vaguely identified as part of the basisphenoid. It has a relatively small exposure in ventral view and a more substantial one in the area of the sphenorbital recess. The ventral exposure contacts the pterygoids, petrosals, and basioccipital. The orbital exposure is in contact with the alisphenoid and the anterior lamina, and possibly the maxilla. 
The ventrally exposed basisphenoid is subtriangular with a smooth surface and lacks any evidence of carotid foramina. The basisphenoid, as part of the mosaic formed by the pterygoids, alisphenoid, and petrosal, is involved in the formation of the posteromedial wall of the lateral palatine trough. Concomitantly, these elements shape the enormously excavated posterior expansion of the lateral palatine trough, but their individual contributions are unclear.

\section{Petrosal}

The petrosal, or parts thereof, are preserved in both specimens. When viewed aneriorly, it is obvious that both skulls have been somewhat deformed from left to right. This makes observation of the left portion of the ear region, in particular, problematic but helps to expose the right sides very satisfactorily. In addition, the right squamosal and part of the petrosal are missing in PSS-MAE 141 and 142, helping observation (Figures 25, 26).

In therian mammals, the petrosal can be divided into two main portions: the pars cochlearis, housing the organs of hearing, and the pars canalicularis, housing the organs of equilibrium and balance. Breakage through the petrosal exposes some of these components of the inner ear in both skulls. A third element, the anterior lamina, is described here jointly with the petrosal. Among living mammals, monotremes are the only group presenting this structure (Kermack and Kielan-Jaworowska, 1971; Griffiths, 1978; Zeller, 1989). In monotremes, the anterior lamina results from the ossification of the lamina obturans, which fuses at various stages of development with the endochondral petrosal (Presley and Steel, 1976; Griffiths, 1978; Presley, 1981; Kemp, 1983; 
Kuhn and Zeller, 1987; Zeller, 1989; Hopson and Rougier, 1993). Although the presence of an anterior lamina has been widely documented among basal mammaliaforms, thus far no sutural distinction between the anterior lamina and the petrosal proper has been identified. In multituberculates, these ossifications seem to be continuous. However, without developmental evidence, it cannot be made certain that the anterior lamina and the petrosal are separate ossifications. Though, considering the evidence afforded by monotremes, it is most parsimonious to assume a similar fundamental pattern in multituberculate mammals. Since the petrosal proper and the anterior lamina cannot be strictly differentiated, and in order to be consistent with treatment of this area by other authors, the anterior lamina will be described as part of the petrosal (See Rougier and Wible, 2006 for a review).

In lateral view (Figures 13,14), the petrosal contacts the sphenoid anteriorly and medially, the frontal anteriorly, the parietal dorsally, and the squamosal posteriorly and laterally. In occipital view, the petrosal contacts the exoccipital and the supraoccipital medially, while contacting the squamosal dorsally. In ventral view (Figures 6, 9, 16, 17, 18, 19, 20), the petrosal contacts the exoccipital in the middle ear recess, basioccipital medially, basisphenoid, alisphenoid and pterygoids anteriorly, and squamosal laterally.

None of the specimens show the endocranial surface of the petrosal and, therefore, its description will be limited to three views: lateral, ventral, and occipital. In ventral view, the most distinctive feature is the enormously excavated middle ear cavity. This cavity has an approximate volume of $4 \mathrm{~mm}^{3}$ (average of 
four measurements of the left ear region of PSS-MAE 141, other ear regions are too damaged to provide reliable estimates). The middle ear cavity is formed mostly by the petrosal with sizeable contributions from the alisphenoid, basioccipital, and exoccipital. The limits of the middle ear cavity are defined by tall crests that approach each other and form a partial, but fairly extensive, floor for this cavity. Deeply recessed in this middle ear space, and occupying an approximate central position in it, is an elongated and ventrally bulging prominence, the promontorium. Several crests at the front and back of the promontorium connect it with other structures of the middle ear. Nevertheless, the main axis and bulging of the promontorium reflect the morphology of the finger-like cochlea, which is partially exposed on the right side of PSS-MAE 141. The three-dimensional arrangements of these crests connecting the promontorium to other structures is best preserved in PSS-MAE 142 because the specimen has sustained less damage to this area. The anterior pole of the promontorium forms a sharp crest that extends ventrally to form an extremely long and robust process, which, on its medial side, is continuous with the middle ear cavity and, on its lateral side, is grooved and is in communication with the epitympanic recess. This very peculiar process in Mangasbaatar is produced by elaboration of the rostral tympanic process of the petrosal (RTPP), which is present in other multituberculates like Kryptobaatar (Wible and Rougier, 2000). However, its extreme dorsoventral elongation results from a ventral projection of the basioccipital and anterior portions of the petrosal in order to accommodate a grossly enlarged middle ear cavity. The recess of the promontorium and medial 
margins of the epitympanic recess might also be factors in enlarging the middle ear space and in determining the unusual morphology of the middle ear of Mangasbaatar.

The groove on the RTPP opens ventrally at the likely junction of the pterygoids, basioccipital, and petrosal. This notch would accommodate the internal carotid artery (ICA). The ICA leaves no other traces of its presence on the skull base, but it was dorsoventrally oriented and tightly pressed to the lateral wall of the RTPP. The broad ICA groove bifurcates at the level of the promontorium, sending a branch posteriorly and a larger one anteriorly. The larger branch excavates a recess in the bony floor of the epitympanic recess. This recess leads to a canal located between the medial edge of the epitympanic recess and the crest marking the dorsal-most extent of the RTPP. The opening of this canal is the internal carotid foramen, well-shown in the right side of PSSMAE 141 and 142. A small fragment of bone is in the ICA foramen in PSS-MAE 142 , obstructing the view of the ICA canal further into the bone. The ICA foramen is formed solely by the petrosal, but a slender posterior process of the alisphenoid, already mentioned, reaches its proximity (this is best shown on the right side of PSS-MAE 141). The condition in Mangasbaatar is different than that presumed to be primitive for members of Djadochtatheriidae, like Kryptobaatar, where the posterior opening of the carotid canal is jointly formed by the petrosal and the alisphenoid before reaching the deeper lying basisphenoid (Wible and Rougier, 2000). Kielan-Jaworowska et al. (2005:499) illustrated the carotid foramen in Catopsbaatar as exposed ventrally and in a very different position 
than in Kryptobaatar and Mangasbaatar, however in their description they mention that the "carotid foramina have not been preserved" (Kielan-Jaworowska et al., 2005:500). The posterior extension of the deep lateral palatine trough extends posteriorly to the edge of the petrosal formed by the RTPP and the carotid foramen.

The posteriorly directed groove originating from the ICA corresponds to the stapedial artery. This extremely rostral position for the stapedial artery is unusual for mammals in general (Wible, 1987), but is actually the condition present in all LCMM for which this area is known. This is also probably the case in most other multituberculates given that is also present in Lambdopsalis (Miao, 1988). The groove for the stapedial artery is proportionally small and runs along the lateral aspect of the promontorium and ventral to the level of the epitympanic recess. The stapedial groove is shallow in all of the specimens and runs along the ventral edge of the fenestra vestibuli. In the vicinity of the fenestra vestibuli, the direction of the groove is ambiguous when considering whether the stapes was columelliform or bicrurated. After traversing the fenestra vestibuli, or running in its vicinity, the stapedial artery is directed towards the common canal for the ramus superior and the prootic sinus.

At the posterior end of the promontorium, there are two fenestrae, one of them laterally positioned (the already mentioned fenestra vestibuli) and the other one medially positioned (the perilymphatic foramen). The fenestra vestibuli can be observed only on PSS-MAE 142 because those of 141 are obscured. The fenestra vestibuli is subcircular and is hardly recessed, if at all, in a fossula 
fenestra vestibuli. The stapedial ratio (Segall, 1970) is 1.3 (average of right and left side of PSS-MAE 142). The fenestra vestibuli is separated from the perilymphatic foramen by a narrow, posteriorly trending bony ridge, the crista interfenestralis (Wible et al., 1995; Rougier et al., 1996a, b). The crista projects ventrally to the body of the promontorium and marks the ventral-most extension of the inner ear. The back of the promontorium and the crista interfenestralis is damaged on the right side of both specimens. The crista, well-preserved on the left side of PSS-MAE 142, remains very tall behind the promontorium and partially subdivides the middle ear cavity into two areas: the first area, lateral to the promontorium, is formed mostly by the epitympanic recess. The second, more medial area, is developed around the jugular foramen and the portion of the middle ear created by contributions of the exoccipital, basioccipital, and petrosal.

The medial portion of the middle ear cavity is further subdivided into two realms, an anterior and a posterior one, by a low crest that extends posteromedially from the edge of the perilymphatic foramen. The posterior portion includes the perilymphatic foramen, the jugular foramen, and the exoccipital contribution to the middle ear space. The perilymphatic foramen is lodged in a deeply-excavated recessus scalae tympani. The edges of the perilymphatic foramen are best seen on the right side of PSS-MAE 142 and appear to be sharp and particularly well developed on the medial aspect of the foramen. The perilymphatic foramen is oriented posteromedially in its direction with respect to the jugular foramen. The large recessus scalae tympani has its major axis oriented in the same direction. The recessus itself extends to the edge 
of the jugular foramen. On the right side of PSS-MAE 142, the anterodorsal border of the recessus scalae tympani is marked by a small crest that extends from the vicinity of the perilymphatic foramen to the jugular foramen.

A second crest, in conjunction to the before mentioned crest, determines a deep groove identified here to have contained the perilymphatic duct. This groove, the perilymphatic sulcus, is almost transformed into a canal by the closely approaching crests mentioned above. It is unsettling, however, to notice that on the left side of the same specimen (PSS-MAE 142), there are no traces of the perilymphatic sulcus, and instead, a poorly developed bulge in the petrosal runs in a similar direction to the groove. The conspicuous absence of the groove on the left side suggests that the perilymphatic duct has been fully enclosed in bone and transformed into a cochlear aqueduct. It is likely that the open groove on the right side is an artifact; the edges of the crests show some breakage, though other portions seem natural. The left side of PSS-MAE 141 (the only side observable for this feature) agrees with the left side of PSS-MAE 142 in showing no traces of a perilymphatic groove. In monotremes the development of the aqueductus cochlea (or lack thereof) differs between Ornithorhynchus and Tachyglossus, as shown by the collection of monotremes housed at the American Museum in New York (AMNH 252512, AMNH 200255, AMNH 157072). The platypus retains a perilymphatic duct mostly open to the middle ear space throughout life (Zeller, 1989), but the echidna closes it later in development. Zaglossus has, in general, an even more complete enclosure of the perilymphatic duct than the echidna. It is possible that a similar situation 
happened in Mangasbaatar, but the fact that its development seems to be different on left and right sides of the same specimen is problematic.

The area of the middle ear cavity developed between the perilymphatic groove, the jugular foramen, the hypoglossal foramen, and the crests subdividing the medial portion of the middle ear cavity is slightly concave and essentially featureless. The petrosal forms at least half of the jugular foramen, which is equal in size to the fenestra vestibuli. The presence of a large jugular bulb in Mangasbaatar is evidenced by the large depression surrounding the jugular foramen and its endocranial enlargement. A portion of the middle ear, anterior to the crest that subdivides the medial portion of the middle ear cavity, forms the bulk of the middle ear volume and determines a concave median surface for the petrosal by excavating all the elements forming the middle ear roof. The RTPP forms the anterolateral limit of this space.

The petrosal extends laterally and posteriorly from the promontorium to form what can be roughly described as an "L"-shaped platform. The long arm of the "L" is formed by the epitympanic recess, and the short one is formed mostly by the caudal tympanic process of the petrosal (CTPP). The epitympanic recess extends from its contact with the alisphenoid anteriorly to the level of the fenestra vestibuli posteriorly. The posterior-most portion of the epitympanic recess is lost from the right side of both specimens and is distorted/incomplete on the left side of both of them. The epitympanic recess is a strongly concave surface that projects ventrally, especially at its anterior pole as it continues into the alisphenoid. Its lateral limits are marked by the ventral edge of the anterior 
lamina and its medial boundary is formed by a low crest that runs posteromedially and that approaches the promontorium quite closely, especially at the level of the fenestra vestibuli. Foramina are present in the petrosal contribution to the epitympanic recess. The most anterior one, already mentioned in connection with the alisphenoid, is oval, anteroventrally directed, and placed approximately along the midline of the epitympanic recess. A second, much smaller foramen, seen only on the right side of PSS-MAE 142, is near the lateral edge of the epitympanic recess and is also anteroventrally directed. A third, of very large size, is centrally positioned in the epitympanic recess, roughly at the level of the internal carotid sulcus. Leading anteroventrally from this foramen, there is a broad, deep sulcus that nearly extends to the anteroventral limits of the epitympanic recess. The three aforementioned foramina of the epitympanic recess likely transmitted branches of the mandibular division of the trigeminal nerve $\left(V_{3}\right)$. The first two foramina are in turn associated with a third foramen that perforates the anterior lamina of the petrosal in lateral view. The three of them can be considered the foramen masticatorium, with two branches directed ventrally and one ventrolaterally.

The big foramen occupying a central position in the epitympanic recess is the foramen ovale inferium. In other multituberculates (Kielan-Jaworowska, 1970, 1971; Kermack and Kielan-Jaworowska, 1971; Kielan-Jaworowska et al., 1986; Hurum, 1988b; Wible and Rougier, 2000) and in some rodents (Hill, 1935; Wahlert, 1974, 1985) the mandibular division of the trigeminal nerve is also divided into numerous foramina. Variations in the number, pattern, and size of 
these foramina seem to be very frequent (Wible and Rougier, 2000), but in PSSMAE 141 and 142 the morphology in this area is consistent. Behind the foramen ovale inferior, the epitympanic recess is walled laterally by the ventral extension of the anterior lamina and at least partially by the squamosal. This region is best preserved in PSS-MAE 141. The medial limit of the epitympanic recess becomes confluent, in this area, with a low crest: the crista parotica, which increases in size posteriorly.

Lateral to the crista parotica, there is a small portion of the epitympanic recess that is deeper than the surrounding area that likely lodged the articulation between the incus and malleus. Therefore, this specific portion of the epitympanic recess is here interpreted as the fossa incudis, for the short crus of the incus. On the left side of PSS-MAE 141, there is a fragment of a small bone, most likely a middle ear ossicle, lying in the fossa incudis. Unfortunately, it is not possible to exactly identify the element. The fossa incudis is fully continuous anteriorly with the epitympanic recess and its posterior limit is formed by the squamosal and the petrosal jointly.

The crest marking the medial limit of the epitympanic recess is connected in the anterior two-thirds of its length to the promontorium. The anterior-most portion of this connection is occupied by the groove for the internal carotid artery. The posterior portion of this connection is almost flat and is notched, only slightly, by the groove for the stapedial artery. The posterior one-third of the crest marking the medial edge of the epitympanic recess is free: the rostral continuation of the crista parotica. At the level of the posterior margin of the 
fenestra vestibuli there is a small process arising from the medial aspect of the crista parotica, which is ventromedially directed, the tympanohyal. Behind the tympanohyal, there is a shallow notch, the stylomastoid notch, which corresponds to the exit of the hyomandibular branch of the facial nerve (CN VII) from the tympanic cavity.

A series of structures occupies the space between the epitympanic recess and the fenestra vestibuli. Posterior to the bony shelf, already described as connecting the epitympanic recess, the petrosal, and the promontorium, there is a deep recess that excavates the lateral surface of the promontorium. This recess marks the likely site of attachment of the $m$. tensor tympani and is identified here as the tensor tympani fossa. The tensor tympani fossa in Mangasbaatar seems to be proportionally small in relation to the size of the middle ear as compared with Kryptobaatar (Wible and Rougier, 2000).

In other LCMM, the facial nerve opening into the middle ear and the prootic canal opening into the middle ear are found behind the tensor tympani fossa. This area is preserved on the left side of PSS-MAE 141 and 142. Unfortunately, preparation of this area is not possible due to the distortion of the skull and the deeply recessed position of these openings. Presence of these structures, namely the prootic canal and the tympanic opening of the facial nerve, is evidenced on the broken right side of PSS-MAE 141. In this specimen, directly rostral to the vestibular structures and lateral to the promontorium, there are two canals shown in section through the anterior lamina. The most medial of these corresponds to the facial foramen, and the more lateral canal corresponds to the 
prootic canal. The prootic canal can be traced posterodorsally to the vicinity of the subarcuate fossa on the internal surface of the petrosal in PSS-MAE 141. A broad facial groove extends posterolaterally, parallel to the promontorium and directed towards the stylomastoid notch. This groove is jointly limited by the squamosal and petrosal. The stylomastoid notch occupies a deeply recessed space between the root of the squamosal, the styloid process, and the tall paraoccipital process of the petrosal. Medial to the stylomastoid notch, at the base of the prominent crista interfenestralis, there is an oval depression: the stapedial fossa. This fossa, subequal in size to the fenestra vestibuli, served as an attachment point for the $m$. stapedius and is hidden in posteroventral view by the prominent paraoccipital process.

The paraoccipital process, in occipital view, is subtriangular with a broad, round apex. A sharp crest extends anteroventrally from the tip of the paraoccipital process and connects this process with the back of the promontorium. The crest on the paraoccipital process is continuous with the crista interfenestralis of the promontorium. This rather large, anteromedially trending crest partially subdivides the rear of the middle ear cavity and results in the absence of a continuous post-promontorial tympanic sinus. The crista interfenestralis-paraoccipital process crest fully separates the structures of the lateral trough from those in the area of the perilymphatic foramen and jugular foramen.

The crista interfenestralis, the edge of the perilymphatic foramen, and the back of the middle ear cavity separate a small portion of the tympanic cavity 
around the jugular foramen. This is the jugular fossa of other multituberculates (Kielan-Jaworowska, 1986; Wible and Rougier, 2000). The jugular fossa in Mangasbaatar represents another out-pocketing of the massive middle ear cavity. The jugular fossa is dominated by the round ventral bulging of the common ampulla of the posterior and lateral semicircular canals. This structure is very similar to that of Kryptobaatar (Wible and Rougier, 2000). The ampullar prominence determines two deep pits in the roof of the middle ear cavity: a lateral and a medial pit. The lateral pit is walled off medially by the crista interfenestralis and posteriorly by the posterior wall of the middle ear cavity. The medial pit is closely associated with the perilymphatic foramen and shows a very well delimited oval depression immediately posterior to the perilymphatic foramen. This area is similar to the recessus scalae tympani described in monotremes (Zeller, 1989). The excavation of this pocket is not as prominent in PSS-MAE 142 as it is in PSS-MAE 141. Determining the occupant of this space is problematic. The resemblance of this area with the recessus scala tympani could suggest that an expansion of the perilymphatic duct would occupy this area, but it was previously noted that the perilymphatic duct is enclosed in an aqueductus cochlearis throughout much of its length. The lateral opening of this aqueduct is on the medial edge of the perilymphatic foramen. It is possible that the perilymphatic duct expanded out of the perilymphatic foramen in a sac filled with perilymph occupying this recess, which is essentially the condition found in monotremes. Alternatively, in eutherians the secondary tympanic membrane stretches over the fenestra cochlearis (the partial homologue of the perilymphatic 
foramen) and there is no expansion of the perilymphatic system into the middle ear cavity. Under this model the recess in Mangasbaatar would be empty and part of the middle ear cavity. The rest of the jugular fossa is gently concave around the jugular foramen.

From the paraoccipital process there is another crest in the petrosal that extends medially; this is the CTPP, which extends to its contact with the exoccipital immediately lateral to the occipital condyles. The CTPP extends anteroventrally and contributes, along with other elements of the braincase, to the floor of the middle ear cavity.

In lateral view, the anterior lamina is the dominant component of the petrosal and, in an undistorted specimen, would probably completely hide the pars canalicularis and the pars cochlearis of the petrosal. The anterior lamina contacts the parietal dorsally through a fairly horizontal suture that extends rostrally up to the anterior opening of the orbitotemporal canal under the root of the postorbital process. The anterior lamina forms the posteroventral edge of this foramen. Following approximately the direction of the anterior lamina-parietal suture, there is an endocranial groove interpreted here as a space for the orbitotemporal system. The major occupant would be the orbitotemporal artery (Kielan-Jaworowska et al., 1986; Rougier et al., 1992; Wible and Rougier, 2000), likely accompanied by a vein. The orbitotemporal artery was fed posteriorly through a fairly vertical dorsal ascending canal. This canal runs between the anterior lamina and the squamosal and is shown by the right side of PSS-MAE 142. In the specimen, the overlying squamosal is missing, affording a direct view 
of the inside of the ascending canal. When complete, the canal would have been perforated by a few foramina that would distribute its contents to the temporal area. The foramina would be occupied by temporal rami feeding the temporal musculature (Rougier et al., 1992; Wible and Gaudin, 2004).

The ascending canal contacts two other grooves: a larger posterodorsal one and a somewhat smaller anteroventral one. The posterodorsal canal opens on the occiput through the posttemporal canal and likely transmitted the arteria diploetica magna and a companion vein (Kielan-Jaworowska et al., 1986; Rougier et al., 1992). The anteroventral canal is filled with matrix in PSS-MAE 142 and represents the ventral ascending canal, likely transmitting a fairly horizontal portion of the ramus superior. This canal, horizontally directed, runs through the thickened ventral edge of the anterior lamina: the lateral flange. The ventral extent of the lateral flange is mostly complete on the left side of PSS-MAE 141. The lateral flange extends anteriorly along the lateral edge of the epitympanic recess as a gently concave lamina. At its most anteroventral extent, near the suture with the alisphenoid, there is a foramen that faces ventrolaterally: the lateral opening of the foramen masticatorium, which has already been mentioned. On the left side of PSS-MAE 141, there is a small foramen on the lower third of the anterior lamina, corresponding to the supraglenoid foramen of Kryptobaatar (Wible and Rougier, 2000) and other LCMM.

The anterior margin of the anterior lamina has a long anteroventrally trending ridge that forms the lateral wall of the sphenorbital fissure. The very long anteroventrally-extending portion of the anterior lamina is one of the most 
conspicuous features distinguishing Mangasbaatar from other LCMM. The extension of the anterior lamina is coupled with the general enlargement of the middle ear cavity through the ventral projection of the elements involved in its formation. The dorsal portion of the anterior edge of the anterior lamina is moderately concave and faces forward as in other LCMM. In Mangasbaatar, this surface is proportionally more dorsally located. The reorganization of this area is mostly due to the very large opening of the sphenorbital fissure, which occupies the floor of the orbitotemporal region. The size and proportions of this area are very well shown by PSS-MAE 142 and at least partially confirmed by the right side of PSS-MAE 141.

In occipital view, the petrosal is exposed between the squamosal, exoccipital, and supraoccipital. The mastoid exposure of the petrosal supports, dorsolaterally, the base of the lambdoid crest. Along the dorsolateral edge of the petrosal there is a fairly prominent posttemporal foramen, partially obliterated by crushing in PSS-MAE 141. This anteroventrally directed foramen is formed solely by the petrosal. A broad groove extends ventrally from the posttemporal canal, continuing into the mastoid exposure of the paraoccipital process. The posttemporal foramen is also slightly notched dorsally suggesting that a large occipital artery ran in close contact with the occiput and continued dorsally to supply the nucal musculature. The area of the mastoid exposure of the petrosal medial to the posttemporal canal is slightly concave and forms a shallow depression on the side of the occipital condyles. This shallow depression 
probably accommodated the transverse processes of the atlas during skull movements.

\section{Basioccipital}

The basioccipital is preserved in both specimens, although only the one in PSS-MAE 142 is incomplete. The basioccipital forms most of the basicranial axis; it is in contact anteriorly with the basisphenoid, laterally with the petrosals, and posteriorly with the exoccipital. It is also possible that a small portion of the pterygoids contacts the anterolateral corner of the basioccipital. In ventral view the basioccipital shows a deep odontoid notch, as described earlier, and a narrow median exposure that is flanked by the exoccipital. In front of the exoccipital, the basioccipital expands laterally contributing to the floor of the middle ear cavity through a thin lamina. Further rostrally, the basioccipital contribution to the floor is in contact with a similar lamina of the petrosal. At the rostral end of the basioccipital there is a transverse suture, mentioned earlier in the description of the pterygoid, that separates the basioccipital from the basisphenoid and probably the pterygoids. The contribution of the basioccipital to the floor of the middle ear is laminar and ventrally convex, similar to the processes of other bones delimiting the middle ear cavity.

In the middle ear cavity, the basioccipital is deeply excavated medially so that this bone forms not only a substantial part of the floor of the middle ear cavity, but also the medial wall and part of the roof. The medial excavation of the basioccipital results in a bone that is very thin, so much so that it can be transilluminated from the opposite middle ear space. This very close 
approximation of right and left middle ear cavities to the midline is ventral to the braincase proper because, as mentioned above, only a thin, tall crest of the basioccipital separates right and left cavities. The suture with the petrosal runs deeply in the roof of the middle ear, obliquely from the jugular foramen towards the anteromedial pole of the middle ear recess. The basioccipital, the petrosal, and possibly the exoccipital, jointly form the jugular foramen. The aperture is of a relatively small size with respect to the overall size of the skull.

\section{Exoccipital}

The exoccipital is incomplete in both specimens. The left side of PSSMAE 141 is the best preserved. The exoccipital contacts the basioccipital anteriorly, the supraoccipital dorsally, and the petrosal laterally and dorsally in the occiput. The exoccipital forms the occipital condyles, most of the foramen magnum, and the posteromedial wall of the middle ear cavity.

Only part of the left condyle is preserved in PSS-MAE 141. What is preserved resembles the condyles present in other LCMM. The condyles are not very prominent, are moderately convex, and have a small lateral extension. The articular surface extends over the bony floor of the middle ear cavity. Right and left exoccipitals are separated ventrally along the midline by a wedge of the basioccipital bearing the odontoid notch. The exoccipital forms most of the lateral edge and floor of the foramen magnum and right and left are separated ventrally, as mentioned before, by the basiocciptal and dorsally by the supraoccipital. The contribution of the exoccipital to the occiput is limited to the area immediately surrounding the foramen magnum. This lamina is flat and slopes slightly forward, 
away from the foramen magnum. The suture with the petrosal runs obliquely, from slightly above the level of the foramen magnum downwards to the medial side of the paraoccipital process in the middle ear cavity.

Mangasbaatar has an extremely well developed and expansive middle ear cavity formed jointly by the squamosal, petrosal, basioccipital, alisphenoid, and exoccipital. The exoccipital forms the posteromedial aspect of this cavity providing a thin floor that extends anterolaterally. This floor is incomplete in both specimens. From what remains of the left side of PSS-MAE 141, it is clear that this floor almost completely enclosed the middle ear. The contribution of the exoccipital to the internal surface of the middle ear can be evaluated only on the right side of PSS-MAE 141. The exoccipital is deeply excavated medially forming a small infundibulum separated anteriorly from the main middle ear cavity by a sharp crest.

In the posteroventral corner of both sides of PSS-MAE 142, a single hypoglossal foramen can be recognized. The area surrounding this foramen is damaged on both sides, so it is unclear if Mangasbaatar had only one hypoglossal foramen or more. A second foramen, piercing the substance of the exoccipital, can be seen on the right side of the exoccipital and part of the same foramen is preserved on the left side. The left exoccipital is more eroded than the right so that the above-mentioned foramen can be seen to be continuous with a cylindrical endocast of the left side. These foramina were inside a condylar area of the exoccipital and can be interpreted to be part of a condylar canal. The endocranial structure leading to the left condylar canal is congruent with similar 
endocasts described for Kryptobaatar (Wible and Rougier, 2000). The relatively large size of these endocasts and the proportionally small size of the jugular foramen imply that most of the blood from the cranial venous system was drained in Mangasbaatar through the foramen magnum instead of the jugular foramen, a condition congruent with that reconstructed for other multituberculates (KielanJaworowska et al., 1986; Rougier et al., 1992). The basioccipital-exoccipital suture in the middle ear region cannot be discerned in any of the specimens so the lateral extent of the bone cannot be ascertained with any certainty. However, it is likely that the relatively large hypoglossal foramen, present on the posteromedial corner of the middle ear cavity, is completely enclosed in the exoccipital (shown by the right side of PSS-MAE 141).

\section{Supraoccipital}

The supraoccipital is a flat bone positioned sagittally on the occiput dorsal to the foramen magnum and is vertical, or inclined slightly forward. The supraoccipital is at least partially preserved in both of the specimens. This element contacts the parietals dorsally, the petrosals parasaggitally and the exoccipitals ventrolaterally. A small portion of the supraoccipital forms the dorsalmost portion of the foramen magnum. In PSS-MAE 142 there are remnants of a small midline crest, which increases in size dorsally. The supraoccipital provides support to the lambdoidal crest but does not directly contribute to it and it is therefore limited to the occipital aspect of the skull. 


\section{Mandible}

Fragments of both right and left lower jaws are preserved in both specimens. Those of PSS-MAE 142 were found dissociated among the numerous fragments that resulted from erosion of the skeleton. The jaws of PSSMAE 141 were still articulated to the skull when found. The right jaw was almost completely eroded away, leaving only the tip of $\mathrm{i} 1, \mathrm{p} 4-\mathrm{m} 2$, and the apex of the coronoid process in articulation. An impression of the body of the jaw was present so that the shape and curvature of the area of the dentary surrounding $i 1$ could be observed. Preparation of the specimen removed all of these remnants of the right jaw and destroyed the natural mold of the dentary. However, the specimen was molded before preparation and the relative positions of the elements are preserved in a rubber cast of the original. The left lower jaw is largely preserved but is missing the symphysis, most of the incisor, and the back

of the jaw. This description is based on this jaw and is supplemented with details from PSS-MAE 142.

The jaw of Mangasbaatar is heavy and deep under the molars with a very steep diastema and i1 alveolus. The i1 is very high and almost reaches the level of the molars. The space between i1 and p3 is relatively short. The symphysis is very robust, as shown by the fragments of PSS-MAE 142. The bottom part of the body of the jaw is missing in both specimens, but can be observed in the cast of PSS-MAE 141. The external surface of the jaw is marked by a series of depressions. The first depression is ventral to the $\mathrm{p} 4-\mathrm{m} 1$ embrasure and has the masseteric fovea preserved as a gentle concavity. Between the masseteric fovea 
and $\mathrm{p} 4$, the alveolar ridge is slightly concave and continues along the chipped teeth to end directly medial to the coronoid process. The anterior portion of this depression under p4 is the lunule (Gambaryan and Kielan-Jaworowska, 1995), which is not very well developed in Mangasbaatar. The more substantial depression extending back is the temporal groove. As previously mentioned, the bottom of the jaw is not well preserved in the specimens and the masseteric crest cannot be fully recognized in any of them, but isolated fragments suggest the presence of the masseteric protuberance (Kielan-Jaworowska et al., 2005). The masseteric fossa, however, is slightly concave and not very large, restricted to the base of the coronoid process, and probably expanding toward the back of the jaw. The coronoid process in Mangasbaatar is high, narrow, and forms a sharp angle with the molariform occlusal plane (78 degrees). The tall coronoid process resembles that described in Catopsbaatar (Kielan-Jaworowska, 1974; Gambaryan and Kielan-Jaworowska, 1995; Kielan-Jaworowska et al., 2005).

Only the dorsal-most portion of the condylar process is preserved in the left lower jaw of PSS-MAE 141. Fortunately, most of the condylar articular surface remains intact. The condyle is perched in a broad neck and is separated from the coronoid process by a wide notch that places the condyle above the occlusal plane of the molars. An isolated left condyle is also preserved in PSSMAE 142. The articular surface is teardrop-shaped with the broad end directed anteriorly. The articular surface continues back along the posterior edge of the jaw for a short distance beyond the broader portions of the condyle, indicating the potential for a large range of jaw opening. The medial view of the jaw shows 
a deep pterygoid fossa that extends anteriorly up to the m2. The pterygoid fossa is bordered anteriorly by the remnants of the pterygoid ridge, which is only partially preserved. At the anterior pole of the pterygoid fossa there is a large foramen that is directed anteriorly into the substance of the dentary: this is the mandibular foramen or canal. All of the features of the medial view of the jaw described by Gambaryan and Kielan-Jaworowska (1995) cannot be described in these specimens.

\section{Dentition}

Mangasbaatar is clearly a member of Djadochtatherioidea (Rougier et al., 1997; Kielan-Jaworowska and Hurum, 1997; 2001) and, as seen in all members of this group, the lower dentition is reduced to one incisor, two premolars, and two molars. The upper dentition is reduced, like Tombaatar and Catopsbaatar, to two incisors, three premolars, and two molars. Therefore, the dental formula for Mangasbaatar is I2/1, C0/0, P3/2, M2/2.

PSS-MAE 141, the type, is a younger individual than PSS-MAE 142. The crown morphology of PSS-MAE 141 is very well-preserved and most of the cusps are intact. PSS-MAE 142, on the other hand, shows extensive wear, which has reduced the cusps to their truncated bases. On the left side of PSS-MAE 141 , there is a small element in the diastema between P1 and P3. This element is a tooth or parts thereof which might be incompletely preserved, but it seems more likely to be part of a deciduous tooth (likely a DP3) which is retained at this ontogenetic stage. The relevant area on the right side of PSS-MAE 141 is damaged, so the presence or absence of this element cannot be corroborated. 
There is, however, a fragment of a tooth in a position that approximately corresponds to the element mentioned above but the damage to the area is so extensive that a positive identification is not warranted. PSS-MAE 141 is a young adult, but the older adult, PSS-MAE 142, shows no trace of this element between $\mathrm{P} 1$ and $\mathrm{P} 3$.

\section{Upper Dentition}

The upper dentition is fairly well-preserved in PSS-MAE 141, although most of the $\mathrm{I} 1$ are missing, the left $\mathrm{I} 2$ and right $\mathrm{P} 4$ are damaged, and the right $\mathrm{P} 1$ P3 are missing. On the skull PSS-MAE 142, only part of the right and left I1 are preserved. Both of the 12 , as well as the right $\mathrm{P} 1$ and $\mathrm{P} 4$, are damaged. The left $\mathrm{P} 1-\mathrm{P} 4$ and the right $\mathrm{M} 1$ are also missing.

INCISORS: The upper I1 is a big, strong tooth that is sharply curved, and occupies most of the premaxilla. The tooth is strongly compressed mediolaterally with a thick layer of enamel covering its lateral aspect, wrapping around the dorsal aspect of the tooth to extend into the medial face (restricted enamel). The medial extension of the enamel is limited to a small dorsal band that occupies less than a third of the medial height of the tooth. Right and left I1 were medially directed and probably contacted each other in the midline. This, however, cannot be seen in the specimens because of imperfect preservation.

The left 12 of both skulls seem to be complete and show heavy wear on their occlusal facets. The 12 in Mangasbaatar is a cylindrical tooth that is slightly curved posteriorly, occupying a position close to the midline on the palate. The I2 
projects ventrally as a long freestanding tooth that culminates into a flat subhorizontal wear facet. The wear of the 12 crowns is enough to obscure the original crown morphology of this element. It is, therefore, uncertain if the 12 had one or more cusps (a primitive condition). The wear on the $\mathrm{I} 2$ is so extensive that the boundary between the crown and the root is obliterated. The wear has also exposed, in the center of the cylinder, a small area showing the cementum surrounded by dentin. This condition is well developed in PSS-MAE 141, but in PSS-MAE 142 the wear on the 12 crown does not reach the cementum.

PREMOLARS: PSS-MAE 141 bears a well-preserved left P1 which shows little wear. The P1 is biradiculated, short, and has a short crown that culminates in three conical cusps. The cusp formula is $1-2$. The cusps are arranged in a closed triangle. The roots are unequal, the anterior root being larger and sloping backwards, a feature quite common among LCMM, likely associated with the palinal masticatory movements (Wall and Krause, 1992; Gambaryan and KielanJaworowska, 1995).

The P2 seems to be absent in Mangasbaatar. No traces of it remain on both sides of PSS-MAE 142. The alveolar bone in this area is well preserved, showing a smooth, short diastema between P1 and P3. On PSS-MAE 141, however, the left side shows the remnant of a tooth between P1 and P3. As preserved, this remnant is little more than a rootlet. It is likely that this is a transitory remnant of a deciduous premolar, likely DP3. The right side of the same specimen is not preserved well enough to be certain of the presence or absence of a P2. Under this interpretation, Mangasbaatar shares the absence of 
a P2 in the adults with Tombaatar and Catopsbaatar. All these large size LCMM represent a closely related group of multituberculates (Kielan-Jaworowska and Hurum, 1997; Rougier et al., 1997). Kielan-Jaworowska et al. (2005) described that in old Catopsbaatar specimens P1 and P3 disappear without leaving traces of their respective alveoli. This does not seem to be the case in Mangasbaatar as attested by the relatively old PSS-MAE 142 which still preserves P1 and P3.

The P3 is a small subrectangular tooth in occlusal outline, with two roots that slant posteriorly. The crown is separated by a strong neck from the roots and bears four conical cusps. The cusp formula is $2-2$. The four cusps of the P3 occupy the corners of a rectangle with a small posterior broadening of the crown behind the two posterior cusps. The posterobuccal and the two lingual cusps are subequal in height, but the anterobuccal is substantially shorter than the other three. The three larger cusps suggest a triangular pattern similar to that present in the P1.

The P4 are missing or badly broken in PSS-MAE 142 and the right side of PSS-MAE 141. The left side of the latter, though, has this element preserved almost completely. The P4 is a large, strong premolar supported by two roots. The cusp formula on the P4 is 1-5-1?. The lingual row is damaged or worn down on both sides of PSS-MAE 141 and it is likely that only one cusp was present; however, it would be possible to have a very small cusp anteriorly positioned relative to the main one. The buccal cusp is small and conical, showing no wear on PSS-MAE 141. The middle row has five cusps that increase in size posteriorly from the first to the fourth, forming a continuous ridge. The fifth cusp is separated 
by a deep embrasure from the rear slope of the prominent fourth cusp. The buccal row, as in other multituberculates, is more dorsally placed than the middle and lingual rows in the P4. The lingual row is likely formed by a single cusp and forms a medially bulging projection which, in essence, represents the abraded base of that large cusp. The wear that obliterated the lingual cusp extends a flat surface to the lingual slopes of the cusps forming the middle row. The buccal bulge formed by the buccal row is offset by the medial bulge of the lingual row, resulting in a slightly oval crown view for the P4 in Mangasbaatar. In Mangasbaatar, as is common among multituberculates, the direction of the middle row is oblique, aligned anteriorly with the lingual row of the P3 but aligned posteriorly with the buccal line of the M1.

The M1 is well preserved in both sides of PSS-MAE 141, but is worn down or broken in PSS-MAE 142. There are three rows of cusps on this molar with a cusp formula of 5-5-3. As is common in other LCMM the buccal and middle rows extend through the length of the tooth, but the lingual row is incomplete. In Mangasbaatar, the incomplete lingual row of the tooth reaches anteriorly to the level of the apex of the third cusp of the middle row, or to the embrasure between the second and third buccal cusps. The elongated M1 is supported by two roots. The anterior root is buccal-lingually compressed and the posterior root, that supports the back half of the tooth, is massive and cylindrical.

The cusps have subrectangular to hexagonal bases and probably culminated in conical apices when unworn. At present, the M1 in PSS-MAE 141 shows small crater-like depressions centrally positioned on each cusp of the 
middle row, reflecting wear through the enamel and exposing the softer dentin. PSS-MAE 142 shows that in older individuals the molars lose almost all relief in their crowns and that the "craters" seen in PSS-MAE 141 expand to occupy the whole crown. The only remnants of the original cusp pattern are in PSS-MAE 142 , seen in the eroded bases of the cusps. The buccal row that is formed by five subequal cusps shows strong wear-facets in all of the lingual slopes. The individual cusps of the buccal row are separated by valleys that become shallower posteriorly; therefore, the first buccal cusp, which is somewhat removed from the anterior margin of the molar, is also separated from the rest of the cusps by the deepest valley. The valley between the second and third cusps of the buccal row is relatively shallow and the bases of these two cusps are partially merged together, a condition that is also present in Tombaatar and other LCMM (Rougier et al., 1997). The fifth buccal cusp is small and poorly separated from the fourth. A blunt ridge extends postero-lingually from its apex towards the last cusp in the middle row, thus closing the trough between the buccal row and other rows. The middle row has five cusps that increase in size progressively toward the back. The cusps in the middle row are positioned at the level of the valleys between the cusps in the buccal row. The imbrication between the cusp bases of the buccal and middle row results in a strongly angular trough between the buccal and middle row. The lingual row has three poorly differentiated cusps, of which the middle is the highest. The bases of these cusps are not as welldeveloped as those of the first two rows and they develop a fairly uniform, 
straight slope that is continuous from the most mesial cusp to the most distal one. Wear facets are developed only on the buccal slope of these cusps.

Both M2 are preserved in both specimens, but those of PSS-MAE 142 are very heavily worn. The M2 is a pear-shaped tooth supported by two roots, a large anterior one and a smaller posterior one that slopes backwards. This position of the roots of the M2 make the occlusal surface of the last molar face anteroventrally, giving the dental arcade a slightly concave outline in lateral view, that is characteristic of multituberculates (Wall and Krause, 1992). The cusp formula of the M2 is ridge-2-3. The two cusps in the middle row are larger than the rest in the molar and continue the posterior increase in size of the middle row of the M1. The bases of these cusps are not polygonal as in the M1. The lingual row is much more prominent in the $\mathrm{M} 2$ than the $\mathrm{M} 1$, forming a fairly sharp ridge that is separated from the middle row by a broad valley. The middle cusp of the three is the largest and has heavy wear as shown by the left molar of PSS-MAE 142. The cusps on the lingual row are very poorly differentiated from one another. The buccal row is very short and does not extend throughout the length of the tooth. It reaches, posteriorly, the middle of the cusp space of the posterior cusp of the middle row. The row lacks distinct cusps and forms a broad, low, shallow crest aligned with the buccal row of the M1.

\section{Lower Dentition}

The best lower dentition is that of the specimen PSS-MAE 141, which is complete (with the exception of the i1). In PSS-MAE 142, only part of the right 
dentition is still in place in a small fragment of the dentary. The left is represented only by isolated broken crowns of the $\mathrm{i} 1, \mathrm{~m} 1$, and $\mathrm{m} 2$.

The first lower incisor is a strong tooth, buccolingually compressed with a partial covering of enamel that, as in the 11 , covers only the external and a small portion of the lingual surface of the tooth. As in all large LCMM, the i1 are very large teeth with an anterior-posterior length subequal to the $\mathrm{m} 1$ length, the largest cheek tooth.

As in all LCMM, Mangasbaatar shows only two premolars, conventionally called p3 and p4. The p3 is, in Mangasbaatar and other LCMM, a very small element, essentially "peg"-like in front of the towering p4. The p3 in Mangasbaatar has only one anteriorly curved root. This root follows the concave contour of the mandibular diastema between $\mathrm{i} 1$ and the p3. There is a clear distinction between the crown and root, indicated by a conspicuous neck. The crown is essentially a more bulbous, enamel-clad continuation of the root that culminates in a single blunt cusp. The apex of the p3 is at roughly the level of the neck between the roots and the crown of the $\mathrm{p} 4$. The anterior margin of the $\mathrm{p} 4$ overhangs the minute $\mathrm{p} 3$ so that the anterior edge of the $\mathrm{p} 4$ is continuous anteroventrally with the mesial edge of the p3. The p3 is well preserved in both the left jaw of PSS-MAE 141 and the right jaw of 142.

The p4 is well preserved in both jaws of PSS-MAE 141, but missing on the left of 142. The p4 in all cimolodont multituberculates is blade-like with a serrated margin. This is also the condition in Mangasbaatar. The p4 in Mangasbaatar is a 
peculiar trapezoidal shape with a nearly straight mesial edge and a fairly horizontal occlusal surface. A similar condition is seen in Catopsbaatar and in Tombaatar (unpublished specimens). The premolar is supported by three roots, two big and stout located mesially and distally and a small one between the two, a condition also known in other LCMM including Kryptobaatar (KielanJaworowska, 1971). The cusp formula in the premolar is ridge-5. The labial ridge is substantial, forming a broad platform on the posterior one-third of the tooth, approximately the area supported by the back root. This ridge becomes broader and stronger posteriorly, reaching the occlusal plane of the buccal row of the $\mathrm{m} 1$ at its posterior extent. The cusps in the central row are very blunt and form a weakly-serrated edge that reaches the level of the occlusal plane of the lingual row of cusps of the $\mathrm{m} 1$.

The first molars (m1) are present bilaterally in both specimens. The best preserved are those in PSS-MAE 141. The $\mathrm{m} 1$ is the largest of the lower cheek teeth, has a cusp formula is $4-3$, and is supported by two massive cylindrical roots. The crown of the $\mathrm{m} 1$ is very low and the cusps of the buccal and lingual rows have different heights (the buccal cusps are lower than the lingual cusps). This difference in height may be exaggerated by differential wear. As is common in other LCMM, the cusps of the buccal and lingual rows occupy alternate positions, with the lingual cusps placed between two successive buccal cusps. The cusps on the buccal side have subhexagonal bases but those on the lingual side are less so. All the cusps in the $\mathrm{m} 1$ were, in the unworn state, conical. 
The $\mathrm{m} 2$ is well preserved and lodged in the dentaries, or fragments thereof, with the exception of the left $\mathrm{m} 2$ of PSS-MAE 142 that was found isolated. The $\mathrm{m} 2$ is a relatively small tooth with only two cusps in the labial and lingual rows.

Therefore, its cusp formula is $2-2$. The crown outline is subrectangular and cusps in both rows are subequal in height. The two rows of cusps are further apart than in the $\mathrm{m} 1$ and, the cusps of different rows do not alternate with each other so that the first cusp of the lingual row is at the same level as the first cusp of the buccal row. The four cusps occupy the corners of the subrectangular m2, determining a broad basin between them. The left m2 of PSS-MAE 142 has been found isolated and it has sustained substantial damage, however, the buccal row is almost complete and shows that the posterior buccal cusp is, in this tooth, subdivided into subequal cusps, a condition not clearly seen on the right $\mathrm{m} 2$ of the same specimen or those of PSS-MAE 141. The $\mathrm{m} 2$ is supported by one root. This root is approximately of the same diameter as the crown and is deeply constricted apically, but is not divided. 


\section{DISCUSSION}

\section{Cladistic Analysis}

Few published phylogenetic analyses include LCMM among a wider sample of multituberculates, most notably Simmons (1993), Rougier et al. (1997), Kielan-Jaworowska and Hurum (1997) and Kielan-Jaworowska and Hurum (2001). Following Rougier et al. (1997) and Kielan-Jaworowska and Hurum (1997) this study recognizes Djadochtatherioidea as a monophyletic group of Late Cretaceous multituberculates and based on shared diagnostic features (see diagnosis) the new taxon will be compared primarily with other LCMM, some of which are found in the same, or similarly aged, sediments. The wider problem of the position of Djadochtatherioidea within Allotheria is not the main purpose of this study; thus I follow Kielan-Jaworowska and Hurum (2001) and KielanJaworowska et al. (2004), regarding the higher-level relationships of Djadochtatherioidea.

To provide a systematic background for the new taxon, I adopt the character and taxon list used by Kielan-Jaworowska and Hurum (1997) with the removal of the unnecessary hypothetical ancestor and the addition of one character for the postpalatine torus. This character is uniquely developed in Tombaatar and Mangasbaatar, and its addition ultimately brings the character number to 45 across 17 taxa, after the addition of Mangasbaatar. 
The resultant data matrix was analyzed using implicit enumeration via Asado version 1.7 which uses TNT as its base searching algorithm. The analysis recovered one most parsimonious tree of 101 steps (ci:0.64 and ri:0.68) which concurs in its structure with that of Kielan-Jaworowska and Hurum (2001) and recovers Djadochtatherioidea as a distinctive taxonomic unit among which the large-sized mutlituberculates including Djadochtatherium, Catopsbaatar, Tombaatar and Mangasbaatar are terminal taxa (Figure 27). These very distinctive mulituberculates reduce the blade-like lower $\mathrm{p} 4$, have very robust jaws, dorsoventrally shallow skulls with small temporal areas but preserve, overall, the typical cimilodontan morphology for the upper and lower molars. Kryptobaatar is a more generalized LCMM and, as a point of comparison, can be interpreted as pleisomorphic within LCMM with regards to the more derived characters seen in Mangasbaatar. Namely, among those, is the development of a very prominent jugular fossa and middle ear cavity, which develops to an unparalleled degree in Mangasbaatar.

The topology of the tree does not identify any clear geographical or stratigraphic pattern. Udan Sayr, where the specimens of Mangasbaatar are from, has traditionally been considered of likely affinities with Barun Goyot from the western Gobi (Szalay and Trofimov, 1996; Kurzanov, 1992). It should be noted, however, that the closest relative of Mangasbaatar appears to be Tombaatar from Ukhaa Tolgod, a locality which is likely to be a Djadochta nearequivalent (Loope et al., 1998; Dingus et al., 2008). Obviously the faunas from 
Ukhaa Tolgod, Barun Goyot and Udan Sayr are all very similar, though, lacking any clearly datable horizons, the relative temporal resolution is somewhat poor (but see Makoviky, 2007).

Four taxa can be considered as large LCMM: they are Djadochtatherium, Tombaatar, Mangasbaatar, and Catopsbaatar. The first two are poorly known, mostly based on palate and jaws, while Catopsbaatar and Mangasbaatar are represented by better preserved specimens. A skull of Djadochtatherium has been utilized in discussing cranial evolution of the large-sized Late Cretaceous Mongolian multituberculates (Kielan-Jaworowska and Hurum, 2001) based on photographs published in reports (sent by Mahito Watabe to Zofia KielanJaworowska in 1996) and popular articles (Webster, 1996). However the specimen has never been thoroughly studied and it is uncertain if it actually is Djadochtatherium. As such there are limited opportunities for comparisons with those bona fide Djadochtatherium specimens recovered by the American expeditions (Simpson, 1925) and deposited in the AMNH (Rougier et al., 1997). It follows that most of what is known about the cranial morphology of these multituberculates is based on Catopsbaatar and now these two specimens of Mangasbaatar. Catopsbaatar has been the focus of a detailed revision by KeilanJaworowska et al. (2005), where a detailed description is provided of the known specimens. All of them, however, have a missing or poorly preserved basicranium. Mangasbaatar, on the other hand, has a relatively complete ear region preserved, with some deformation, in two specimens. It is presently uncertain if the extreme development of sinuses and cavities in the ear region of 
Mangasbaatar is characteristic of all four of these large LCMM. However, in unpublished specimens of Tombaatar, the ear region is also greatly excavated but differs substantially in detail from that of Mangasbaatar. In conjunction with the fact that, in Kryptobaatar, there is already a substantial excavation of the middle ear region (Figure 27), and particularly of the area surrounding the jugular fossa (Wible and Rougier, 2000, Figures 14, 20, 21), it seems likely that at least some degree of middle ear expansion was present in all of these large-sized LCMM. The enlargement of the middle ear cavity would therefore be a synapomorphy of this group that is further elaborated and taken to an extreme.

\section{Discussion of characters}

Rougier et al. (1998), Wible et al. (2004) included the postpalatine torus as a binary character with the states absent (0) and present (1) in an analysis of Zalambdalestes. In order to include the peculiar morphology of the torus seen in Tombaatar and Mangasbaatar, this study adds this character in 3 states; absent or very faint (0), developed laterally and with a venral projection from the palate, forming a distinctive bulge (1), strongly developed, forming a raised, ornate and sharply angled plate (2) (see Figures 32 and 33 for full character list and scoring). This character alone resolves relationships at the most terminal end of the LCMM, placing Tombaatar and Mangasbaatar as sister groups, one node removed from Catopsbaatar.

For character 15, which codes the cusp formula for M1 as either 4-5:4-5:05 (0), 5-7:5-8:2-5 (1) or 5-11:7-10:6-11 (2), Mangasbaatar falls ambiguously 
within character states 0 and 1 , due to its M1 cusp formula of 5-5-2. Therefore, in this analysis, this character has been coded as multistate $(0+1)$. As noted by Kielan-Jaworowska and Hurum (2001) the level of parallelism within multituberculate dental development makes results from their comparison less robust. As such, this character would benefit from further breakdown in future studies which attempt to resolve relationships at these more terminal taxa.

For character 18, which encodes the M2 cusp formula, there exists ambiguity in the inner ridge of the M2 of Mangasbaatar between specimens, with PSS-MAE 141 showing ridge-2-3 and PSS-MAE 142 displaying ridge-2-2, though the M2 of PSS-MAE 142 is heavily worn. Given the character states, 1:2:2 (0), 1:2:3 (1), and greater than 1:2:3 (2), Mangasbaatar is tentatively scored as $0+1$, to account for the apparent ambiguity.

The addition of Mangasbaatar and the postpalatine torus as a character generate a phylogenetic tree (Figure 28) which is highly similar to that in KielanJaworowska et al. (1997), with few exceptions. Differences include the placement of Buginbaatar within a clade with Taeniolabis and Lambdopsalis, the placement of Bulganbaatar with Nemegbaatar as a sister group to the clade containing Nessovbaatar and Kamptobaatar. Similarly to Kielan-Jaworowska et al. (1997), positioning Nessovbaatar is difficult due to the lack of data, and the variable positions of Bulganbaatar and Kamptobaatar in different trees, which in turn affects the affinities of Nessovbaatar. Beside these changes, the monophyly of Chulsanbaatar, Sloanbaatar, Kryptobaatar, Djadochtatherium, Catopsbaatar, Tombaatar and the new taxon Mangasbaatar remains clear. 


\section{Status of Djadochateriidae}

The results of my analysis are consistent with recent LCMM phylogenies (Rougier et al., 1997; Kielan-Jaworowska and Hurum, 1997; 2001) which assign Kryptobaatar, Djadochatherium, Catopsbaatar and Tombaatar to the monophyletic clade Djadochtatheriidae. This family of LCMM is recognized by cranial characters, differing from the remaining members of Djadochtatherioidea in having a subtrapezoidal snout in dorsal view which is confluent with the zygomatic arches, an irregular, non-oval anterior part of the promontorium, in addition to the snout extending for more than $50 \%$ of the skull length (KielanJaworowska et al., 1997). Given the anatomical evidence provided by the two skulls in this study and the resulting cladogram Mangasbaatar is here referred to Djadochtatheriidae. Ultimately, this contribution adds yet another Mongolian taxon represented by well-preserved skull material, which provides details that can potentially serve as a reference for the derived multituberculate morphology characteristic of the large-sized LCMM.

Kryptobaatar is perhaps the best known LCMM (Kielan-Jaworowska, 1971; Wible and Rougier, 2000), with hundreds of skulls known from Ukhaa Tolgod, Mongolia (Dashzeveg et al., 1995; Wible and Rougier, 2000). There are, however discrepancies regarding its relationships within Djadochtatheriidae, mostly due to differences in the characters used in the phylogenetic analyses; Kielan-Jaworowska et al. (1997) placed Kryptobaatar within Djadochtatheriidae in contrast with a cladistic analysis by Rougier et al. (1997) which placed Kryptobaatar between Chulsanbaatar and Bulganbaatar. Kielan-Jaworowska 
reasoned that the character for the subtrapezoidal snout, which is unique for Djadochtatheriidae within all of Mammalia, likely evolved only once, which places Kryptobaatar next to Djadochtatherium, Tombaatar and Catopsbaatar, however Kryptobaatar is similar in size to the smaller LCMM. The cladistic analysis performed here agrees with this placement, thus I hold that Kryptobaatar is currently the most closely related form to these large LCMM multituberculates.

\section{Comparisons with other LCMM}

Highlited here are outsanding features of the dentition and skull of Mangasbaatar in comparison to other LCMM, in a bone by bone discussion where appropriate (See Figures 28, 29, 30, 31 for comparative photographs, and illustrations).

\section{Premaxilla}

In ventral view the premaxilla has thickenings between the alveoli for 12 and I3, a feature which is present in several other forms such as Tombaatar, Nemegbtaatar, Kryptobaatar and Chulsanbaatar, but not present in Kamptobaatar (Kielan-Jaworowska et al., 1986; Wible and Rougier, 2000; Rougier, 1997).

As is the condition for nearly all multituberculates described to date, there is no internarial bar or septomaxilla present in Mangasbaatar. The internarial bar was noted by Miao (1988) in two specimens of Lambdopsalis, though this finding is controversial as both of these bars were of differing size between specimens. Regardless, the feature has no taxonomic utility, as Lambdopsalis is the only 
known multituberculate to express this condition. The condition is currently unknown in Haramiyavia, Arboroharamiyavia and Megaconus (Jenkins et al., 1997; Zheng et al., 2013; Zhou et al., 2013 ) and is absent in other primitive forms, only making an appearance within basal mammaliaformes such as Morganucodon (Kermack et al., 1981), Haldanodon (Lillegraven and Krusat, 1991), and possibly Docodon (Rougier et al., 2014).

\section{Maxilla}

The sub-trapezoidal shape of the skull is due, in part, to the lateral bulging of the maxilla. This bulging is, as indicated by CT scans (Kielan-Jaworowska and Hurum, 1997, 2001; Rougier et al., 1997; Kik, 2002; Kielan-Jaworowska et al., 2004, 2005) an accommodation for an enlarged maxillary sinus. Such a large development of the maxillary sinuses is found uniquely within some members of Djadochtatherioidea, namely Kryptobaatar, Djadochtatherium, Catopsbaatar and Tombaatar (Kielan-Jaworowska \& Hurum, 1997; Wible \& Rougier, 2000; Rougier et al., 1997; Kielan-Jaworowska et al., 2005) and results in a distinctive trapezoidal snout. Other LCMM, ptilodontoids and taeniolabidoids such as Lambdopsalis and Taenolabis, exhibit an arcuate snout which forms a sharp angle with the zygomatic arches at their point of contact (Kielan-Jaworowska \& Hurum, 1997; Miao, 1988).

Like Catopsbaatar, there appears to be a secondary infraorbital foramen present in Mangasbaatar, though the utility of this feature in cladistics has been questioned by Miao (1988) and others as it may be variable within a species. 
Nevertheless, multiple rostral exits for the infraorbital canal is a basal mammaliaform feature present in morganucodontids (Kermack, 1981), some dryolestoids, and Vincelestes (Rougier et al., 1992; Rougier, 1993) in addition to monotremes (Kuhn, 1971; Zeller, 1989).

\section{Palatine}

The bones which contribute to the formation of the "orbital mosaic" are best known in Kryptobaatar and Lambdopsalis, where in both the palatine has no contribution and the maxilla forms the anterior part of the orbital roof. Like these two taxa, Mangasbatar also lacks an exposure of the palatine within the orbital fossa. Miao (1988) speculated that the lack of the palatine within the orbit may be a synapmorphy for multituberculates, which is supported by Lambdopsalis, Kryptobaatar and Mangasbaatar. Interpretations of the orbital fossa of Nemegbaatar by Hurum (1994, 1998a) and illustrations of Ectypodus (Sloan, 1979: fig. 1) noted a palatine exposure within the orbital area which would, if interpreted correctly, suggest that the feature is polymorphic within LCMM. In contrast, Wible and Rougier (2000), though unable to examine some of the reported specimens of Nemegbataar, examined Ectypodus (YPM-PU 14724), and did not identify the sutures suggesting the presence of this exposure, confirming that the same area in Kryptobaatar is formed of maxilla. It appears likely that in LCMM the palatine is excluded from the orbital mosaic or it is at least very small. 
Djadochtateriid multituberculates routinely share a uniquely developed postpalatine torus, most strongly developed in Mangasbaatar. The presence, absence, and relative size of this feature has been scored within Kryptobaatar (Wible \& Rougier, 2000), Tombaatar (Rougier et al., 1997), Catopsbaatar (Kielan-Jaworowska, 2005), Lambdopsalis (Miao, 1988), and Zalambdalestes (Rougier et al., 1998). The postpalatine torus in Tombaatar is strongly developed; however, when compared with that in Mangasbaatar, the central ridge on the torus lacks the shallow recess that the torus of Mangasbaatar exhibits. The torus in Kryptobaatar is weaker than either previously mentioned and also, like Tombaatar, lacks the central recess present in Mangasbaatar. In 2005, a specimen of Catopsbaatar was published in which the torus was completely preserved, specimen ZPAL MgM-I/80, which shows a weakly developed torus. Nevertheless, the post-palatine torus is consistently present among large Mongolian multituberculates.

Though the exact role of the postpalatine torus in extinct and extant mammals remains ambiguous, the degree of development within djadochtatheriids to the exclusion of other multituberculates suggests that it may represent, at least, a useful phylogenetic character. In extant mammals, such as the aardvark or the hedgehog, this bony feature is located at the attachment for the tensor veli palatini muscle (Barghusen, 1986), which tenses the soft palate. It is unclear what benefit a more robust process of this sort confers as modern taxa which share it do not establish a clear pattern among diet, body size and the morphology of the torus. 
Nasal

PSS-MAE 142 retains the posteromost portion of the right nasal, which reveals that the frontals project a wedge in between the suture between the nasals posteriorly, a primitive character present in nearly all Mesozoic mammals, save for perhaps Catopsbaatar, though it is likely that this is due to poor preservation of the sutural pattern in Catopsbaatar (Kielan-Jaworowska et al., 2005).

\section{Lacrimal}

Due to previous interpretations of multituberculate skulls which noted the absence of a lacrimal (Kielan-Jaworowska, 1971) it was argued that this character may be a synapomorphy of multituberculates, strengthening the argument for a close relationship between monotremes and multituberculates. Following the observation of a lacrimal in Nemegbaatar (Kielan-Jaworowska, 1974), this view was revised. Indeed, a lacrimal has since been recognized as characteristic of LCMM, as a large, roughly rectangular bone visible on the dorsal surface of the skull, bordering the frontal from the maxilla (Kielan-Jaworowska and Hurum, 1997). Wible and Rougier (2000) were unable to identify with certainty the presence of a lacrimal in the specimens of Sloanbaatar, Catopsbaatar, or Bulganbaatar stored in Warsaw, though other specimens of Catopsbaatar show the character clearly. They add that a sizable lacrimal is a primitive feature in multituberculates, present in paulchoffatids and LCMM, though the extent of the orbital exposure of this bone was uncertain in other taxa. 
Lambdopsalis (Miao, 1988) lacks the lacrimal completely and Taeniolabis must have lacked a facial exposure of the bone if it were present as claimed by KielanJaworowska and and Hurum (1997). Mangasbaatar fits the condition described for other LCMM, displaying a large, subrectangular lacrimal which separates the frontal from the maxilla on the dorsal surface of the skull. In Mangasbaatar, the lacrimal contributes to the formation of an orbital pocket, as in Kryptobaatar which has a small orbital process (Wible and Rougier, 2000).

\section{Frontal}

The ethmoid foramen in Mangasbaatar is formed by both the frontal and orbitoshpenoid and likely distributed the ethmoid nerve and artery. Though the presence of this foramen was questioned by Simmons (1993) in both Ectypodus and Ptilodus, a more recent analysis by Wible and Rougier (2000) refuted this claim, holding that the character is present, not only in Kryptobaatar but in all multituberculates including Ptilodus and Ectypodus, as well in all living mammals.

\section{Parietal}

The postorbital process is common among mammalian skulls (Novacek, 1986), and its morphology, whether long, short, or composed of frontal, parietal or both has been a point of interest in distinguishing relationships within multituberculates. Miao (1988) held that the postorbital process seen in multituberculates is not homologous to that seen in other mammals because the parietal position of the postorbital process does not delimit the back of the orbit. This claim has, however, been refuted numerous times (see Wible and Rougier, 
2000). Among multituberculates the postorbital process is positioned more posteriorly over the orbit than in other therians and among LCMM this feature is entirely formed by the parietal (Gambaryan and Kielan-Jaworowska, 1995). In Tombaatar, Catopsbaatar Mangasbaatatar and Djadochtatherium, the postorbital process is comprised entirely by the parietal and, contrary to the condition in Chulsanbaatar, Kamptobaatar and Nemegbaatar (Kielan-Jaworowska and Hurum, 1997) is very long (Kielan-Jaworowska and Hurum, 1997; Wible and Rougier, 2000; Kielan Jaworowska et al., 2005), and is best seen on the left side of PSS-MAE 141.

\section{Squamosal}

The exposed region on the right side of PSS-MAW-141 reveals the contact between the squamosal and the petrosal, showing that the squamosal forms the lateral and dorsal walls of the ascending canal. This has been previously described in Kryptobaatar and Vincelestes (Rougier et al., 1992; Wible and Rougier, 2000) and it is a primitive component of the basal mammaliaform circulatory pattern (Wible, 1983, 1986; Rougier et al., 1992, 2006).

\section{Sphenoid}

Mangasbaatar has a sphenorbital fissure, which is assumed to transport V2 and the ethmoidal artery from the cavum epiptericum. Chulsanbaatar, Nemegtbaatar, Kryptobaatar, and Sloanbaatar have all been interpreted as having a separate foramen for CNIII (Rougier et al., 1997; Hurum, 1998b; Wible and Rougier, 2000), dubbed the metoptic foramen, which is unknown in the 
chondrocranium of any living mammal, as they do not co-express the pilae metoptica and antotica.

Anteroventral to the optic foramen, there is another relatively large foramen which penetrates the sphenoid, corresponding to the transverse canal in Kryptobaatar, which is known in some marsupials and placentals to transmit the transverse canal vein (Wible and Rougier, 2000). The specimens of Catopsbaatar described by Kielan-Jaworowska et al. (2005) were not well preserved in this area, making interpretation of foramina or sutures in the area of the orbit uncertain. The phylogenetic implications of this are not clear as the area is not commonly accessible.

\section{Petrosal}

The petrosal is the bony region of the skull with two parts, the pars cochlearis that houses the cochlea, the organ of hearing, and the pars cannalicularis, that houses the vestibular organs. There are four surfaces of this bone: an endocranial, ventral, lateral and dorsal view, although the endocranial portion of the bone in either of the specimens is not exposed.

It is notable that the perilymphatic grooves which differ on the left and right of PSS-MAE 142 present a hurdle in the comparison of these specimens. The left side of PSS-MAE 141 has no evidence of a groove, agreeing with the condition on the right side of PSS-MAE 142, suggesting that in Mangasbaatar, the groove is covered by bone forming an enclosed duct. Despite some variability in this particular feature, the jugular fossa between the two is highly similar. 
Multituberculates, though, have shown some variability in the perilymphatic duct's enclosure by bone. Several isolated petrosals, likely belonging to taeniolabidoids, collected from the Late Cretaceous of North America displayed a condition wherein the perilymphatic duct was not fully bounded by bone, but rather by two bony lappets bordering the groove without making contact with one another (Wible and Rougier, 2006).

Some multituberculates share certain features with monotremes such as a large anterior lamina of the petrosal, and, like adult monotremes, in Mangasbaatar, no clear suture can be seen between the anterior lamina and the petrosal. Given the developmental origin of this feature within monotremes, however, it is parsimonius to assume that a similar origin accounts for the feature within multituberculates. The anterior lamina of the petrosal is lacking in Lambdopsalis but present in Mangasbaatar, further distinguishing Djadochtatherioidea from Lambdopsalis (Miao, 1988; Kielan-Jaworowska et al., 2004) and potentially other taeniolabidoids.

On the ventral view it becomes apparent that the region surrounding the braincase is significantly dorsally depressed posterior to the palatine which produces several exaggerated features such as an enlarged rostral tympanic process of the petrosal. In Mangasbaatar the RTPP is significantly larger in proportion with the rest of the skull when compared with Kryptobaatar, likely owing to the depressed ear region. Within this area, a concave space formed between the RTPP and the basioccipital is composed of both the petrosal and the basioccipital in Mangasbaatar and Kryptobaatar (Wible and Rougier, 2000). 
The two bones both contribute to this cavity; however, in Mangasbaatar, the bordering basioccipital bone which forms the cavity's medial wall has a greater height dorso-ventrally and the contribution of this bone to the aforementioned cavity is nearly vertical in its entirety. This space is further subdivided by crests expanding into the middle ear cavity from the petrosal and the basioccipital; these extensions do not fully divide the region in compartments, but determine specific areas within the cavernous middle ear.

Middle Ear (Petrosal, Basioccipital and Exoccipital)

Due to the acquisition of several derived features, particularly in the ear region, Mangasbaatar appears as a highly specialized member of Djadochtatherioidea, closely related to Tombaatar and Catopsbaatar. Observations of Kryoptobaatar, currently the most thoroughly documented multituberculate, and related members establish a trend among derived members of this group to enlarge the middle ear space. This region in Mangasbaatar is unique among other LCMM in the depth created by a dorsal depression of the basicranium, measured at roughly $4 \mathrm{~mm}^{3}$. It is difficult to compare this specimen to related taxa due to lack of consistent preservation of the region; however it is clear that this cavity is proportionally larger than that of, Kryptobaatar, Catopsbaatar, and based on unpublished specimens, Tombaatar.

In Mangasbaatar, all of the surrounding elements of the ear region project ventrally and help to encompass a very large space. The membranous component of the middle ear cavity likely extended ventrally from the edges of 
those cavities, and it was certainly of sizable proportions. But if compared with other LCMM, the proportion of the cavity delimited by bone is much larger in Mangasbaatar than in any of the other previously described multituberculates from the region. In Kryptobaatar, the same elements - exoccipital, basioccipital, basisphenoid, squamosal, and petrosal — also project ventrally and form distinct pockets but these are not as prominent. The circumscribing of the middle ear region by bony projections in Mangasbaatar is interpreted here as an alternate way of producing a bony encasing of the middle ear region. A similar "sinking" of the petrosal into the braincase can been seen in some borhyenoid marsupials (de Muizon et al., 1997) and the surrounding elements also provide a partial enclosure of the middle ear cavity; in these marsupials, as in multituberculates, the vast majority of the middle ear space was enclosed by a membrane and a fully developed bulla is lacking.

The functional significance of an expanded middle ear region is not straightforward to ascertain. The mammalian middle ear is an impedance transformer that matches the impedance between air-transmitted sound and the perilymphatic fluid in the inner ear (Webster and Webster, 1984). Often, among living mammals, the middle ear cavity is surrounded by a rigid osseous bulla composed by a variety of bony elements that include the ectotympanic as the main support for the tympanic memebrane. The ectotympanic can contribute to the enclosure of the middle ear by forming a substantial portion of the bulla, but depending on the groups, a variety of neomorphic elements or other bones from the basicranium can participate in the formation of the bulla. The specific 
composition of the bulla has traditionally been a source of systematic and phylogenetic data (Novacek and Wyss, 1977). The combination of elements, shapes, sizes, and presence of internal division by septae, etc., of the ear region determine an optimal frequency for a given array of morphologies; this is the natural frequency of a middle ear (Fleischer, 1978). This frequency increases with stiffness of both membrane-ear ossicles and tympanic cavity, but decreases with a greater mass of the membrane (functionally including the ossicular chain) and a larger air volume (Dallos, 1973; Fleischer, 1978; Mason, 2006; Mason, 2013). However, the primitive condition for mammals is the absence of a bulla, and most of the tympanic cavity is surrounded by a membrane that has, on its lateral aspect, a ring formed by the ectotympanic that supports the tympanic membrane. Such a middle ear is susceptible to deformation by chewing and jaw movements that deform the middle ear space and alter its auditory tuning. Additionally, a membrane-encased middle ear region is more susceptible to interference of the low frequency sounds. This primitive mammalian ear is present among monotremes and basal marsupials (Klaauw, 1931; Simpson, 1938; Griffiths, 1978; Ashwell, 2013). Similarly, in Mangasbaatar the middle ear cavity is surrounded only partially by bone and most of its ventral surface must have been enclosed by membrane; therefore it is hard to accept the same degree of stiffness in multituberculates than, for example, in desert dwelling rodents with large middle ear spaces enclosed by a bulla (Webster, 1966; Fleischer, 1973; Mason 2006, 2013). Nevertheless, many of these recent mammals with large middle ear spaces (Mason, 2004; Mason, 2013) spend a 
substantial portion of their lives underground; the environmental conditions in Ukhaa Tolgod and most of the Late Cretaceous Mongolian sites seem to indicate that the environment was dominated by sands and some degree of periodical or seasonal aridity (Loope, 1998; Dingus et al., 2008). In this context, an enlarged, more rigidly enclosed middle ear region would be consistent with an optimization for the perception of low frequency sound by increasing the non-pliable surfaces surrounding the middle ear.

Talpids (moles) and golden moles share a medially sunken middle ear cavity which forms an open connection between the two middle ears through an opening in the basicranium which allows for pressure-difference localization in the low frequency range. Though such an adaptation is common among nonmammalian tetrapods, it is rare among mammals which typically have these two cavities separated by bone and soft tissue. Among the talpids and golden moles the expansion of the middle ear cavity is done by recession of the basicranium bordering the medial wall of the middle ear cavity, while other mammals typically expand their middle ear cavities via ventral expansion of the bulla (Mason, 2013). If the volume of the middle ear spaces of Kryptobaatar and Mangasbaatar are compared, it is clear that the increase is due mostly to the "sinking" of the petrosal and promontorial area into the braincase. Though there is no known open connection between the middle ear spaces of any multituberculate studied thus far, the medial expansion of the middle ear cavity is similar to what must have occurred in the ancestors of golden or talpid moles. 
Furthermore, the tensor tympanii fossa, which is formed via attachment of the m.tensor tympanii, is relatively small in Mangasbaatar in comparison with the rest of the middle ear space. It can be inferred, by extension, that the $m$. tensor tympanii was also relatively small. A similar morphology is noted in the marsupial mole Notoryctes (Ladeveze et al., 2008), while the muscle is completely lost in adult golden moles (Mason, 2003) and a variety of mammals with a fossorial lifestyle (Mason, 2013). Indeed, the tensor tympanii muscle has been lost, convergently, in at least 4 distantly-related groups: marsupial moles, spalacid molre-rats, golden moles and talpid moles, all of which occupy a subterranean habitat (Mason, 2013). The stapedius muscles is also often missing in forms with enlarged middle ear cavities (Hinchcliffe and Pye, 1969; Webster and Webster, 1975; Heffner et al., 2001), however no mammal is known to be missing both muscles, the tensor tympani and the stapedius muscle. The stapedius muscle increases stiffness of the middle ear ossicles and this in turn dampens transmission of low frequency sound. The presence or absence of a stapedius muscle cannot be unequivocally ascertained in Mangasbaatar, however a stapedius fossa has been identified in multituberculates (Kielan-Jaworowska et al., 1986; Rougier et al., 1992, 1996a; Wible and Rougier, 2000) and it appears likely that the muscle was present among LCMM. Meng (1992) described the stapes of Lambdopsalis, the element is well preserved, columelliform, and without a distinct stapedius process. It is therefore possible that in at least some multituberculates the stapedius muscle was much reduced or absent. A partial stapes of Kryptobaatar was described by Rougier et al. (1996a) as bicrurated, 
but the specimen is incomplete and no evidence of a stapedius process is preserved. It is clear that some degree of diversity existed among multituberculates and that the LCMM and Lambdopsalis are both radically different regarding their adaptation related to the ear region. Reports of middle ear bones by Hurum et al. $(1995,1996)$ in some LCMM are hampered by misidentification of calcite concretions as fragmentary ear ossicles and cannot be discussed further until the specimens are restudied.

In summary, such adaptations to the middle ear could confer a number of advantages, as seen in extant mammals such as the golden mole, which is nearly blind, relying on its acute hearing to track prey and avoid predation (Mason, 2003; Webster and Webster, 1975). Though multituberculates were previously assumed to be mostly arboreal, several discoveries have suggested that these creatures fulfilled a greater variety of ecological niches, including a fossorial existence for Lambdopsalis, suggested by Miao (1988), in part, by its expanded ear region, a flat incudomalleal joint, and the apparent lack of a fossa muscularis minor which would, if present, aid in high frequency hearing, an ability which does not appear to be cruicial when living underground (Miao \& Lillegraven, 1986; Miao, 1988; Gambaryan and Kielan Jaworowska, 1995). Applying these assumptions to the paleobiology of djadochtatheriids is still, however, highly speculative. 


\section{Dentition}

The dental formula of both Mangasbaatar specimens (I2/1, C0/0, P3/2, $\mathrm{M} 2 / 2$ ) and their molar and premolar morphology clearly refer these multituberculates to Djadochtatherioidea, and further analysis of their skull morphology and petrosal anatomy refers them as terminal members of Djaadochtatheriidae. Unlike Tombaatar, and similar to all other LCMM, the I3 is placed entirely within the premaxilla, whereas in Tombaatar, the $\mathrm{I} 3$ is in direct contact with the maxilla and premaxilla, lying within the suture between the two bones. Like Tombaatar, the biradiculated P1 has three, conical cusps and the P3 in turn has four conical cusps; all that remains between $\mathrm{P} 1$ and $\mathrm{P} 3$ is a short diastema which likely housed the remnants of the DP3 (Rougier et al., 1997). The best preserved P4 among these two specimens is highly similar to that in Tombaatar, differing in the relative heights of the cusps within the middle row. In Tombaatar, the middle cusp row in P4 contains five cusps, with the third in the row being the tallest of them all (Rougier et al., 1997), in contrast to Mangasbaatar, in which the 5 cusps of the middle row of P4 increase in size posteriorly. Aside from this difference, the overall morphology of this cusp row is highly similar to Tombaatar and other LCMM, aligned posteriorly with the buccal cusp row of M1 and anteriorly with the lingual cusp row of P3 (Rougier et al., 1997).

The M1 cusp formula in Mangasbaatar (5:5:2) differs from Tombaatar (4:5:2), Catopsbaatar (5-6:5-6:4) and Kryptobaatar (4-5:4:3-5), while the M1 is not preserved in the type specimen of Djadochtatherium (Rougier et al., 1997; 
Kielan-Jaworowska and Hurum, 1997; Kielan-Jaworowska et al., 2004; KielanJaworowska et al., 2005). Mangasbaatar most closely resembles Tombaatar in this regard, though the molars are slightly larger within Mangasbaatar. Additionally, in occlusal view, the outer edge of the lingual cusp row in the M1 of Tombaatar, Mangasbaatar, and Kryptobaatar forms a bulge from the remaining body of the tooth. In Catopsbaatar, the outer edge of the M1 lingual cusp row is confluent with the remaining rows in the $\mathrm{M} 1$.

Though the type specimen of Tombaatar is lacking the posterior half of the cranium, preliminary observations of another, more complete (unpublished) specimen of Tombaatar show a high degree of similarity with Mangasbaatar. Despite their similarities, both specimens of Mangasbaatar are slightly larger in comparison with Tombaatar.The M2 on Tombaatar are slightly smaller with respect to Mangasbaatar, and the skull length of PSS-MAE $141(6.30 \mathrm{~cm})$ is greater than that of the unpublished specimen of Tombaatar $(5.91 \mathrm{~cm})$. Though PSS-MAE 141 and PSS-MAE 142 are somewhat deformed, the palatal region and the dental arch of Mangasbaatar can be accurately observed and exhibit less curvature than in Tomabaatar. The dentition of the large members of Djadochtatherioidea retains the same overall morphology of more generalized multitubeculates like Kryptobaatar, where the $\mathrm{M} / \mathrm{m} 1$ is of moderate size and the $\mathrm{M} / \mathrm{m} 2$ remains small but distinctive, while the $\mathrm{P} 4$ is relatively small and uncomplicated. The p4 on the other hand loses the blade-like aspect characteristic of most multituberculates and it it supported mesially by a peg-like 
p3. The trapezoidal outline of the p4 and the absence of a P2 are distinctive dental feature of the large LCMM. 


\section{CONCLUSIONS}

Multituberculates posses the longest-spanning fossil record of any known mammal lineage, extending from as early as the Middle Jurassic to the late Eocene (Kielan-Jaworowska et al., 2004). Reported in this study is a new genus, Mangasbaatar, based on the craniodental description of two specimens, PSSMAE 141 and PSS-MAE 142, from the Late Cretaceous locality Udan Sayr, located in the Gobi Desert of Mongolia, a site which has produced several wellpreserved specimens (Kurzanov, 1992; Dong and Currie, 1993; Trofimov and Szalay, 1996; Rougier et al., 1998).

The skull of Mangasbaatar is highly similar to the closely related Tombaatar, Catopsbaar, Kryptobaatar, and Djadochtatherium. The suite of features including the subtrapezoidal shape of the skull, the dentition, petrosal

anatomy and other features clearly ally Mangasbaatar with these other taxa. The morphology of the postpalatine torus, though unknown in function, is a highly conspicuous character among more derived members of LCMM and is most strongly developed in Mangasbaatar and Tombaatar. Based on the phylogenetic analysis adapted from Kielan-Jaworoska and Hurum (1997), and emended here, this study recovers Mangasbaatar among the djadochatherian LCMM. Specifically, Mangasbaatar is a terminal member of the monophyletic clade Djadochtatherioidea, and sister-group to Tombaatar. 
The degree of excavation of the middle ear cavity in Mangasbaatar, in addition to the relatively small tensor tympanni fossa, may suggest a fossorial or semi-fossorial habit for Mangasbaatar, as this is a pattern seen among modern, fossorial, desert-dwelling rodents (Mason, 2013). The ventral expansion of the bones enclosing this space may have functioned similarly to the bulla in modern mammals, forming a rigid base for the soft structures encasing the middle ear, increasing the rigidity of the cavity thus aiding in low frequency audition (Fleischer, 1978; Mason, 2013), adding to the wide variety of habitats and niches occupied by multituberculates. 


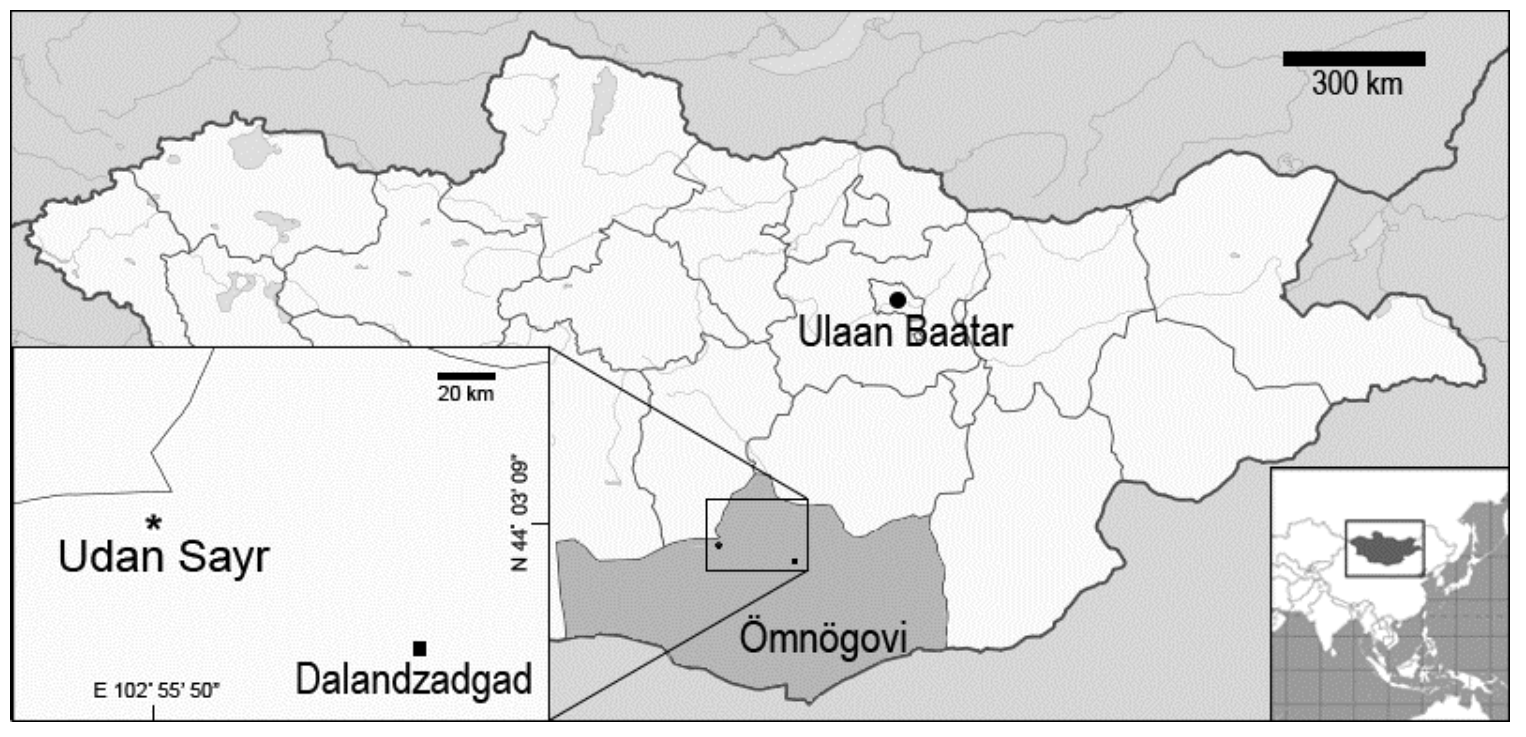

Fig. 1. Map of the Udan Sayr locality of the Gobi Desert in Mongolia. 


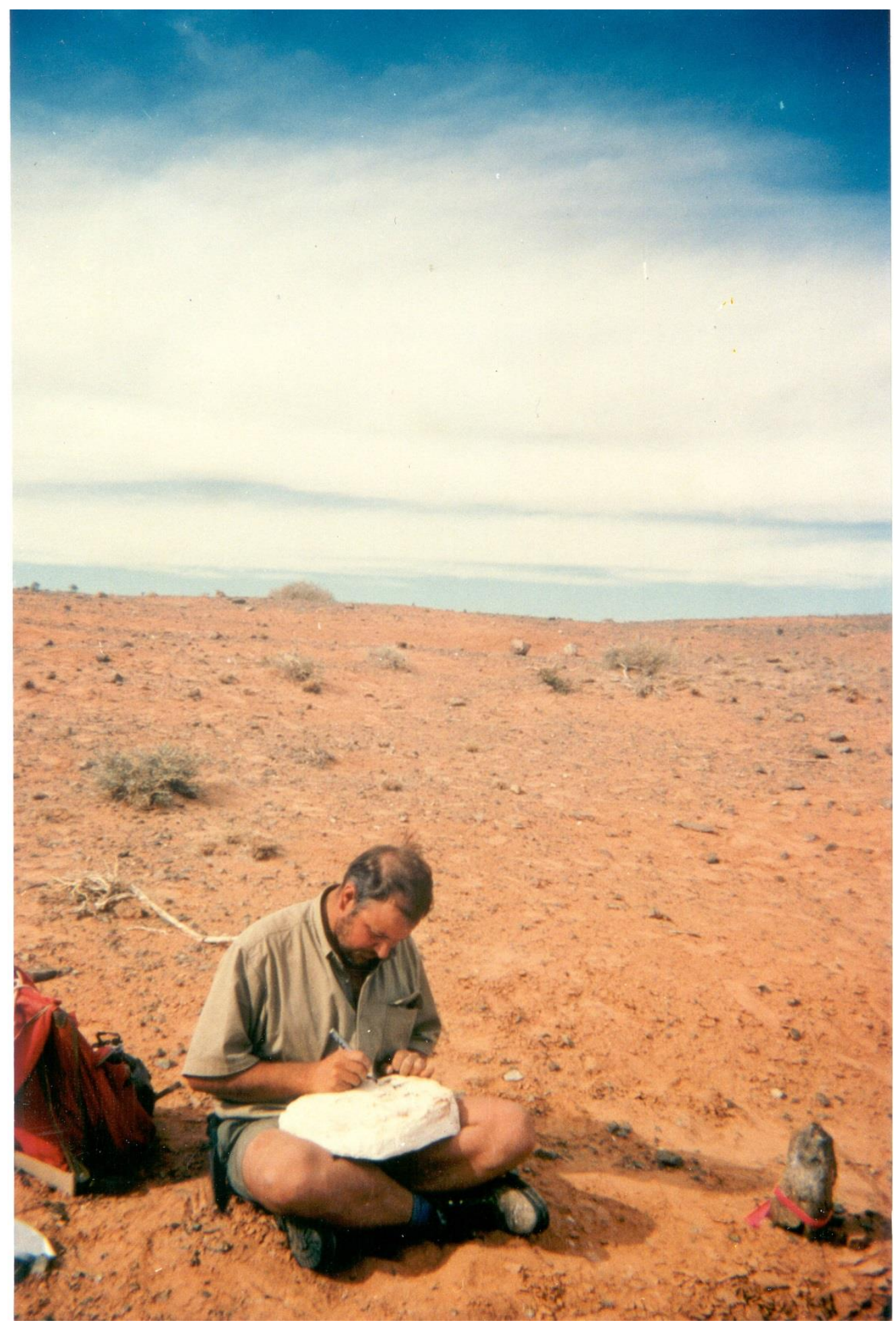

Fig. 2. Photograph of the Udan Sayr locality of the Gobi Desert in Mongolia. 

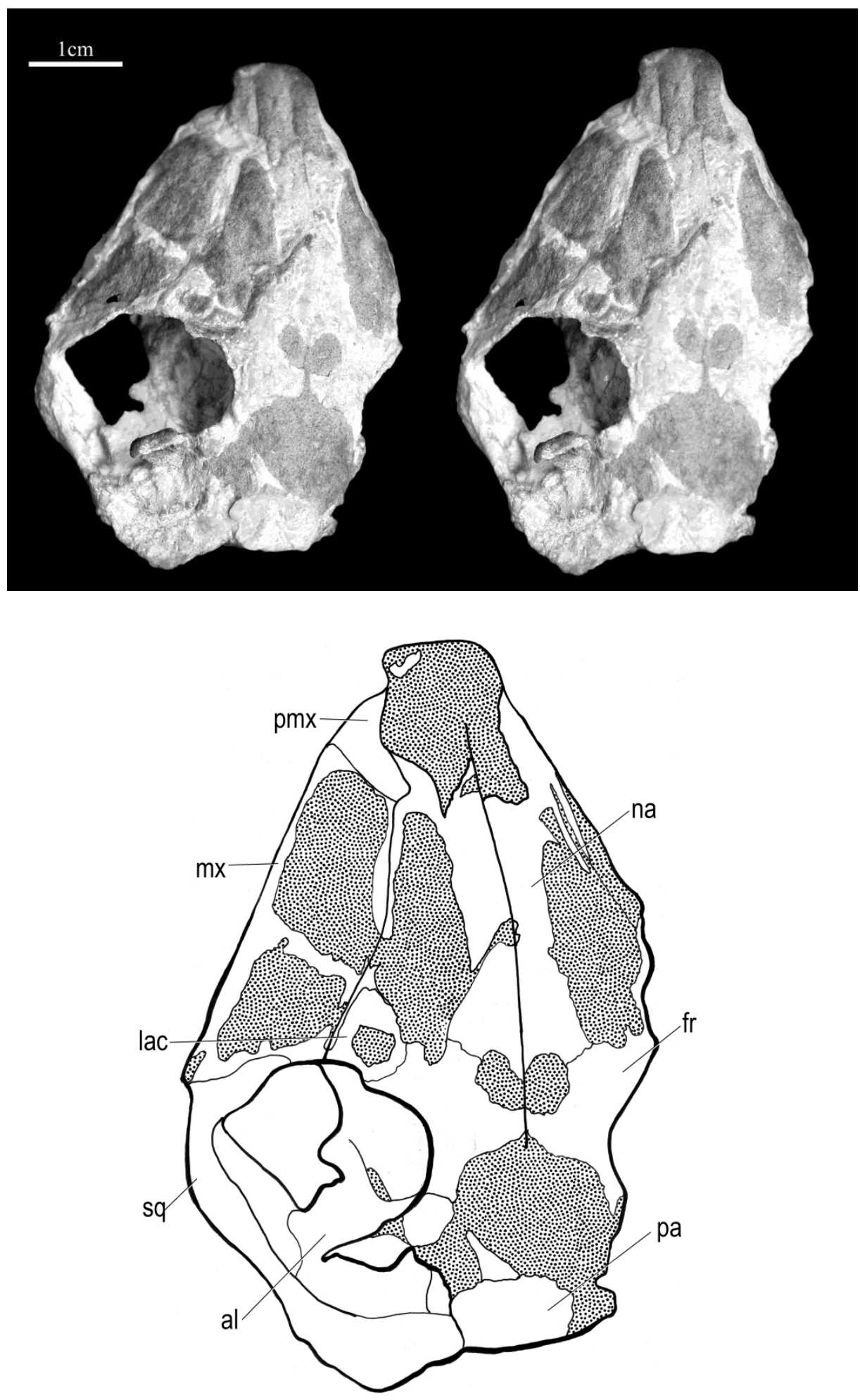

Fig. 3. Stereophotograph of the skull of PSS-MAE 141, holotype of Mangasbaatar udanii, gen. et sp. nov., in dorsal view, with accompanying line drawing. Dotted pattern represents matrix. Abbreviations: al anterior lamina; fr frontal; lac lacrimal; mx maxilla; na nasal; pa parietal; pmx premaxilla; sq squamosal. 

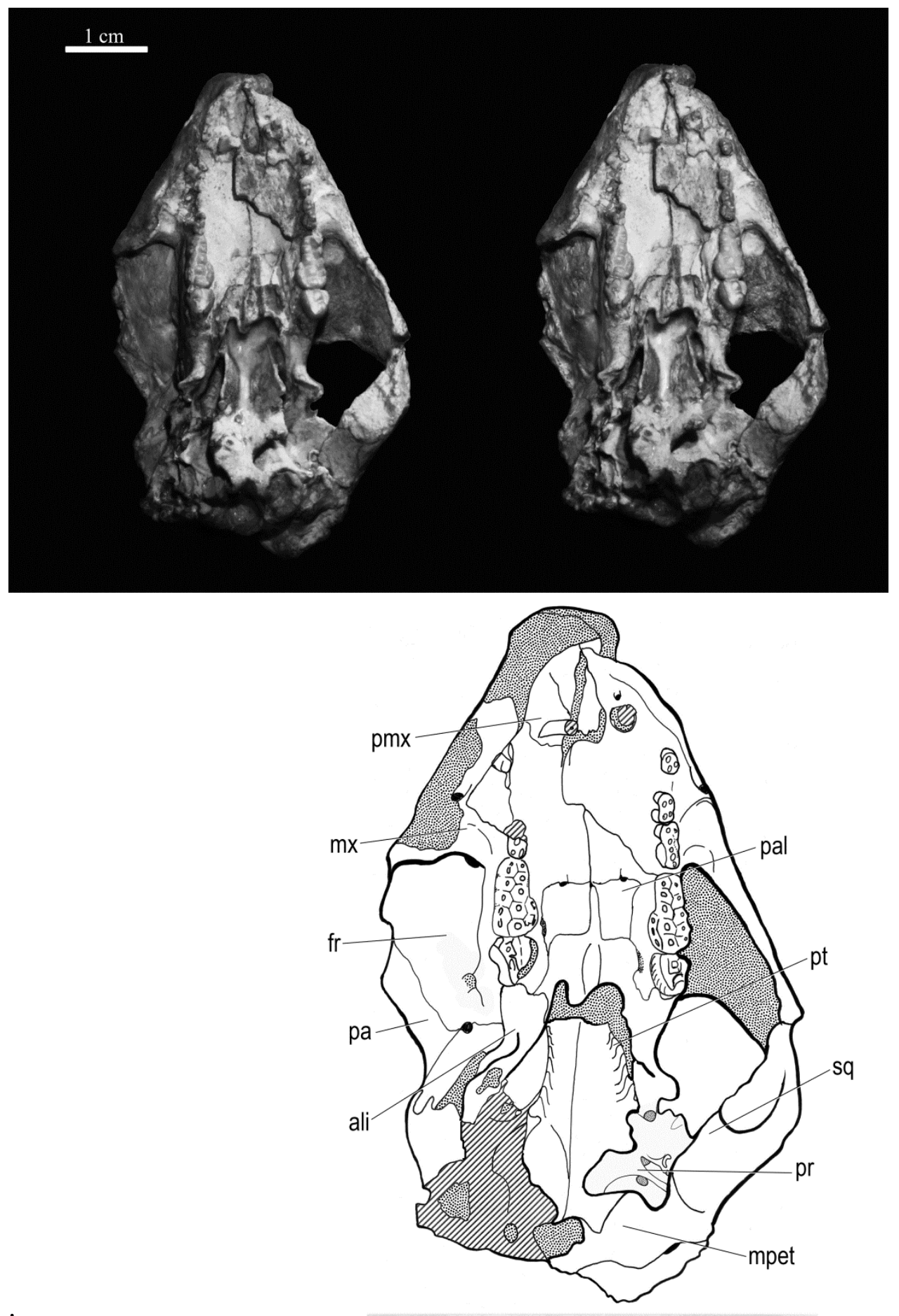

Fig. 4. Stereophotograph of the skull of PSS-MAE 141, holotype of Mangasbaatar udanii, gen. et sp. nov., in ventral view with accompanying line drawing. Dotted pattern represents matrix; parallel lines represent damaged surfaces. Abbreviations: al anterior lamina; exoc exoccipital; fr frontal; lac lacrimal; man mandible; mx maxilla; na nasal; or orbitosphenoid; pa parietal; pet petrosal; pmx premaxilla; sq squamosal. 


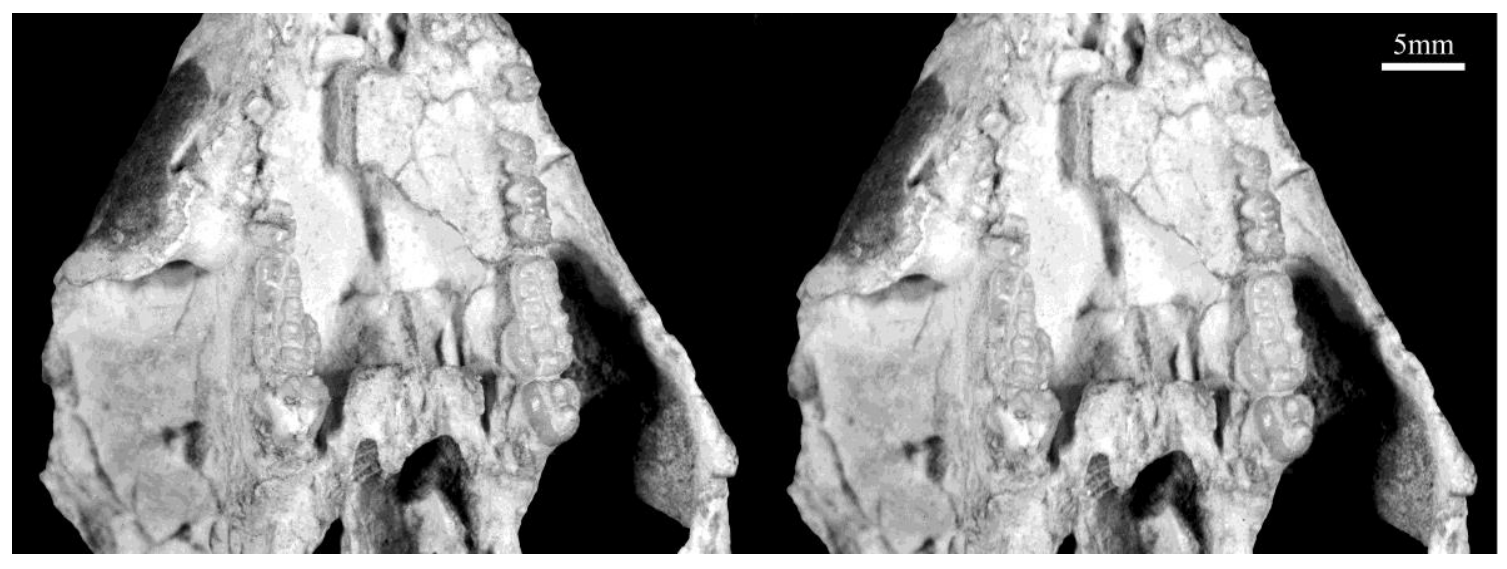

Fig.5. Stereophotograph of the palatal region of PSS-MAE 141, holotype of Mangasbaatar udanii, gen. et sp. nov., in ventral view. 
A.

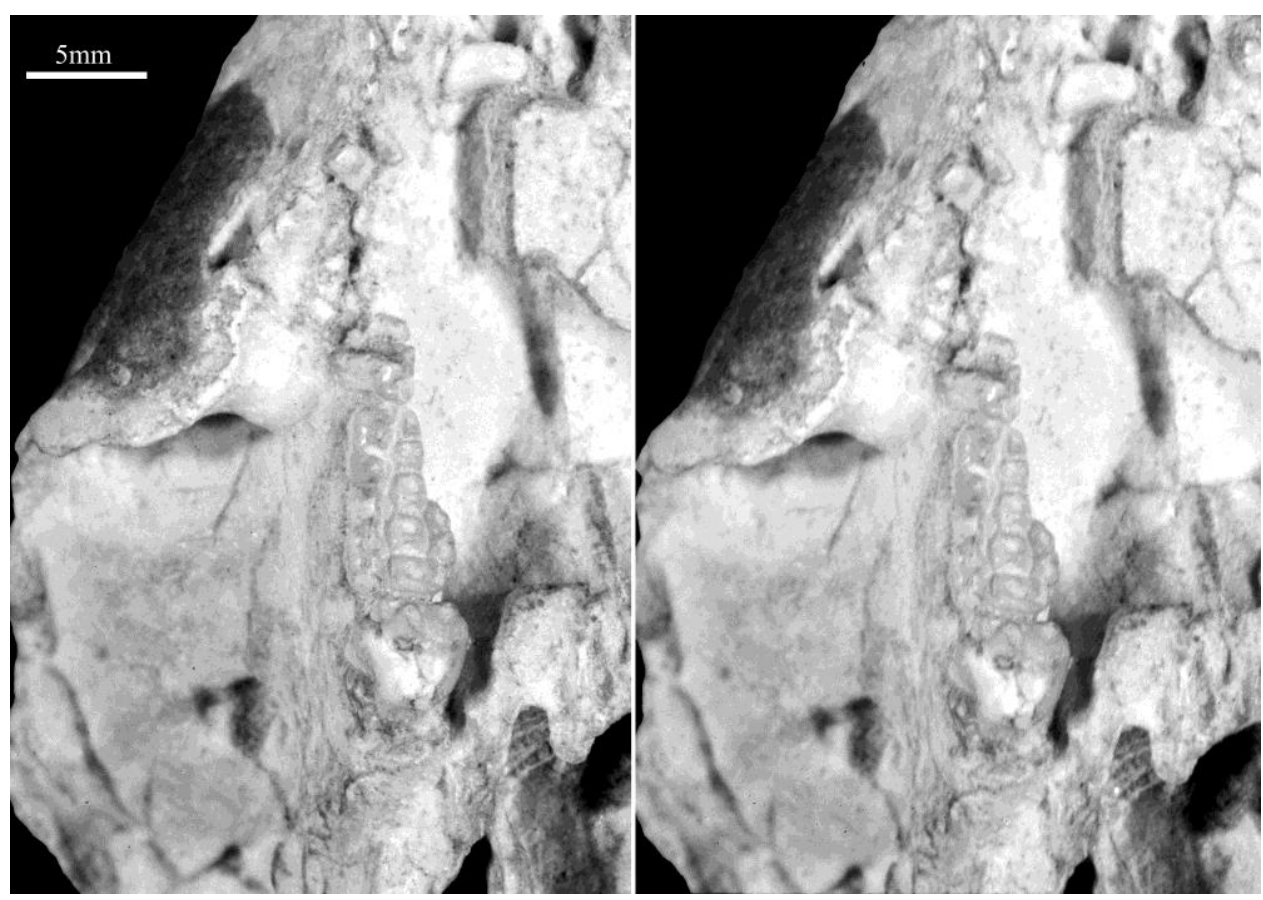

B.

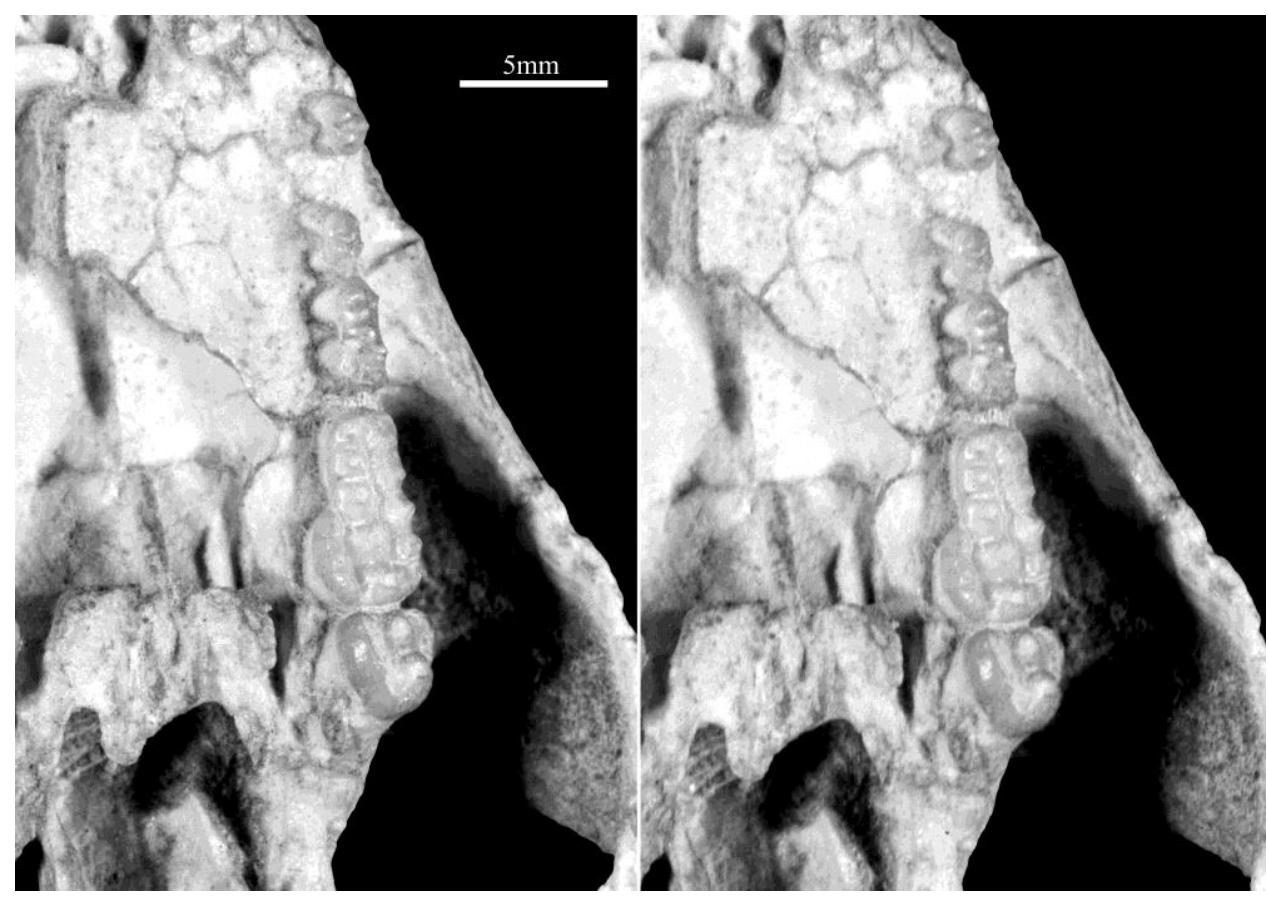

Fig. 6. Stereophotographs of the right and left sides of the palatal region of PSS-MAE 141, holotype of Mangasbaatar udanii, gen. et sp. nov., in ventral view (A) Detailed view of the right half of the skull and (B) a detailed view of the left side of the skull. 


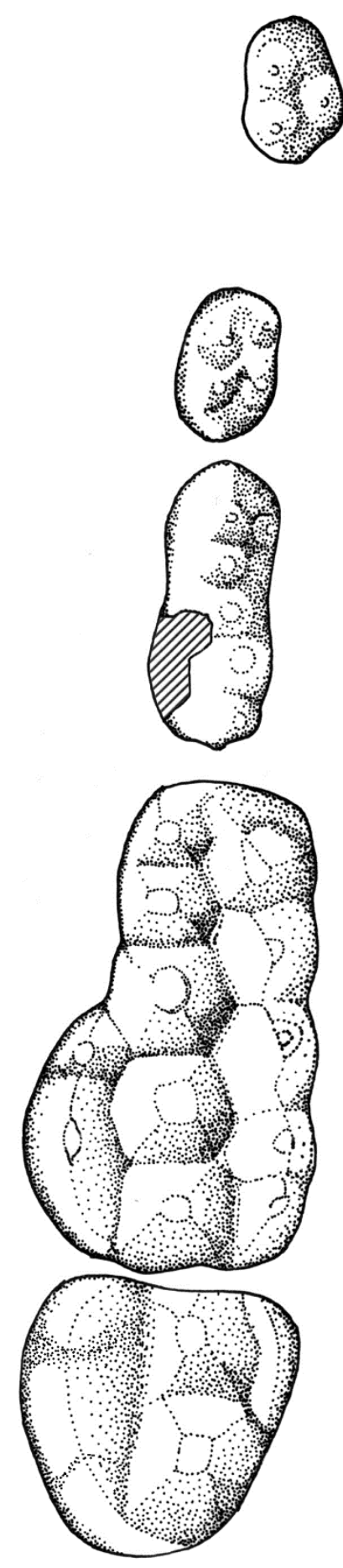

Fig. 7. Ilustration of the left upper dentition of PSS-MAE 141, holotype of Mangasbaatar udanii, gen. et sp. nov., in occlusal view. Parallel lines represent damaged surfaces. 


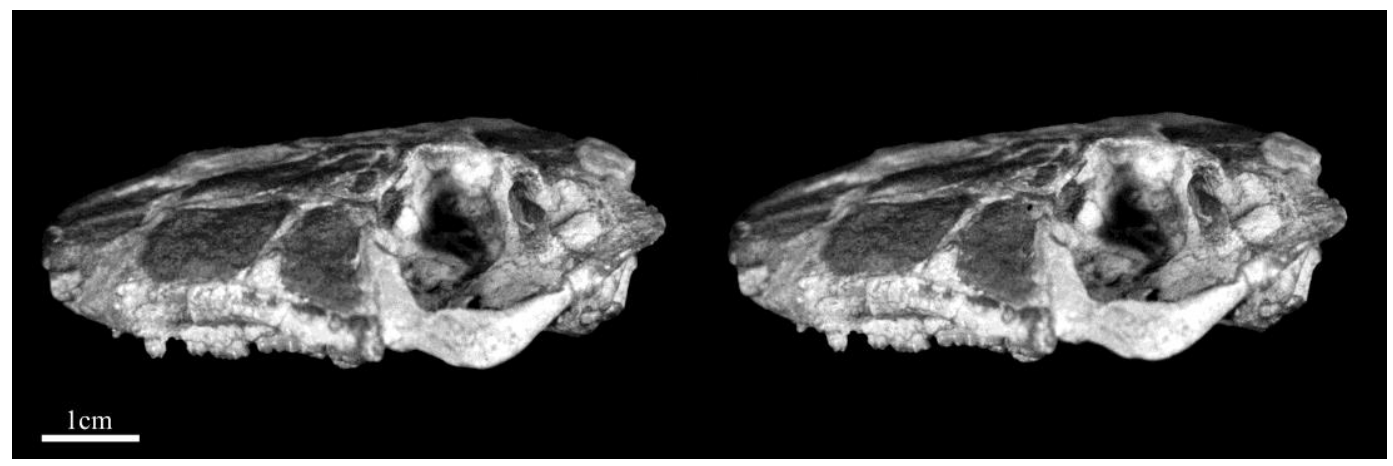

Fig. 8. Stereophotograph of the left side of the skull of PSS-MAE 141, holotype of Mangasbaatar udanii, gen. et sp. nov., in lateral view.

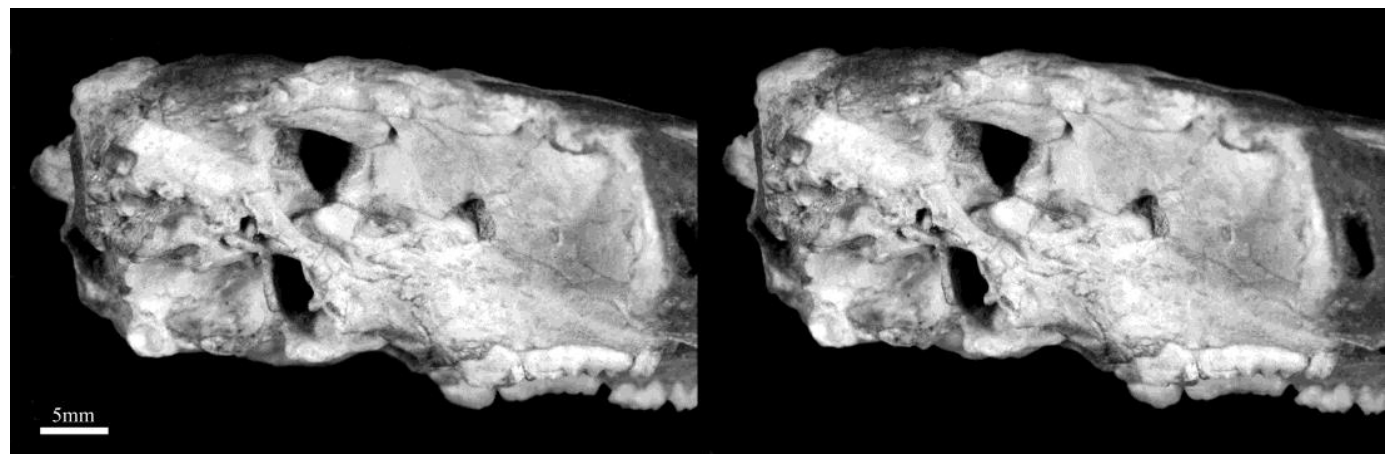

Fig. 9. Stereophotograph of the right ear region PSS-MAE 141, holotype of Mangasbaatar udanii, gen. et sp. nov., in lateral view. 

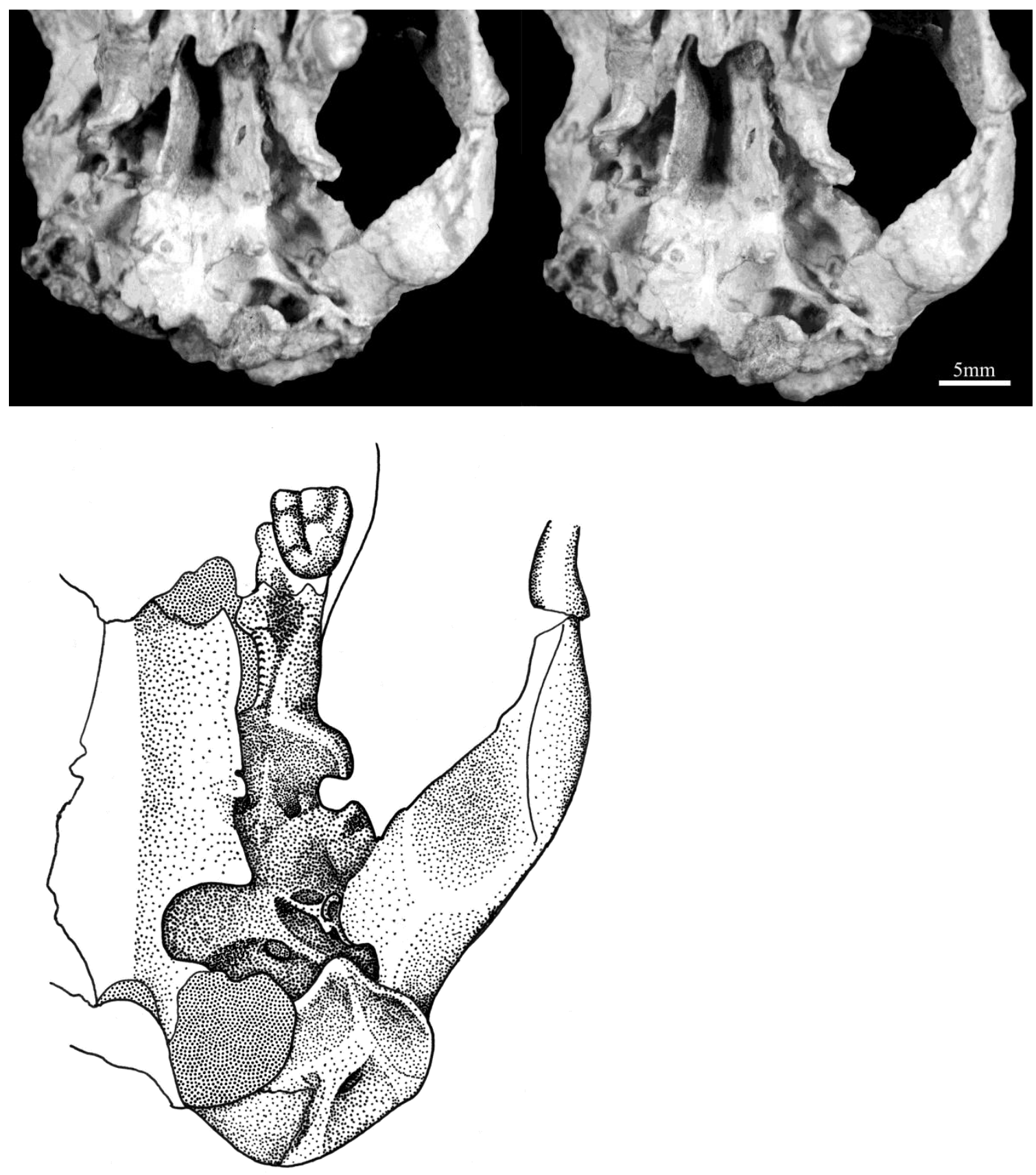

Fig. 10. Stereophotograph of the basicranium of PSS-MAE 141, holotype of Mangasbaatar udanii, gen. et sp. nov., in ventral view with accompanying illustration. 


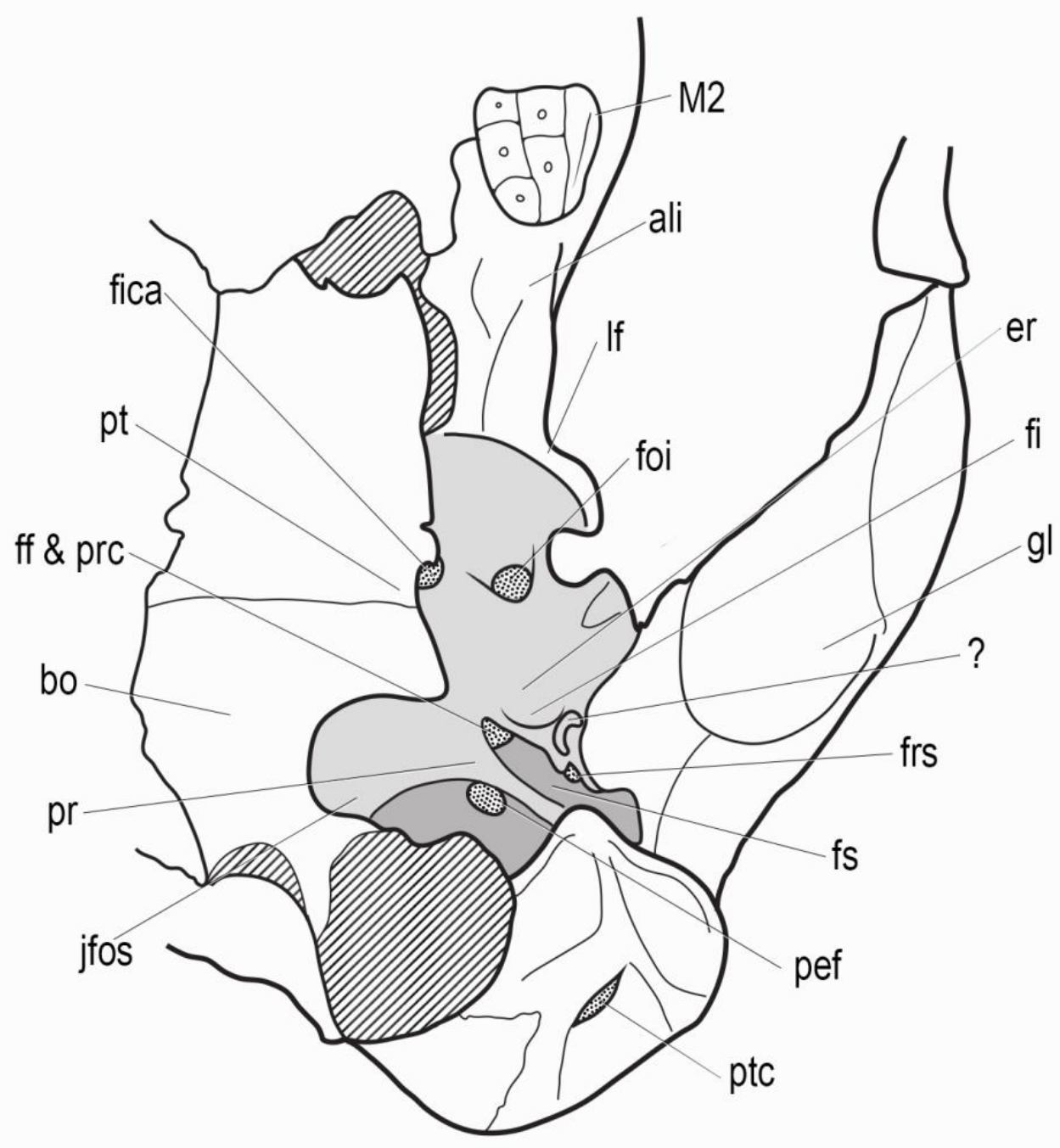

Fig. 11. Line drawing of the basicranium of PSS-MAE 141 in ventral view. Abbreviations: ali alisphenoid; bo basioccipital; er epitympanic recess; ff facial foramen; fi fossa incudis; fica foramen for internal carotid artery; foi foramen ovale inferiorum; frs foramen for ramus superior; fs facial sulcus; gl glenoid; jfos jugular fossa; If lateral flange; M2 upper molar 2; pef perilymphatic foramen; pr promontorium; prc prootic canal; pt pterygoid; ptc posttemporal canal; ? possible fragment of incus. 


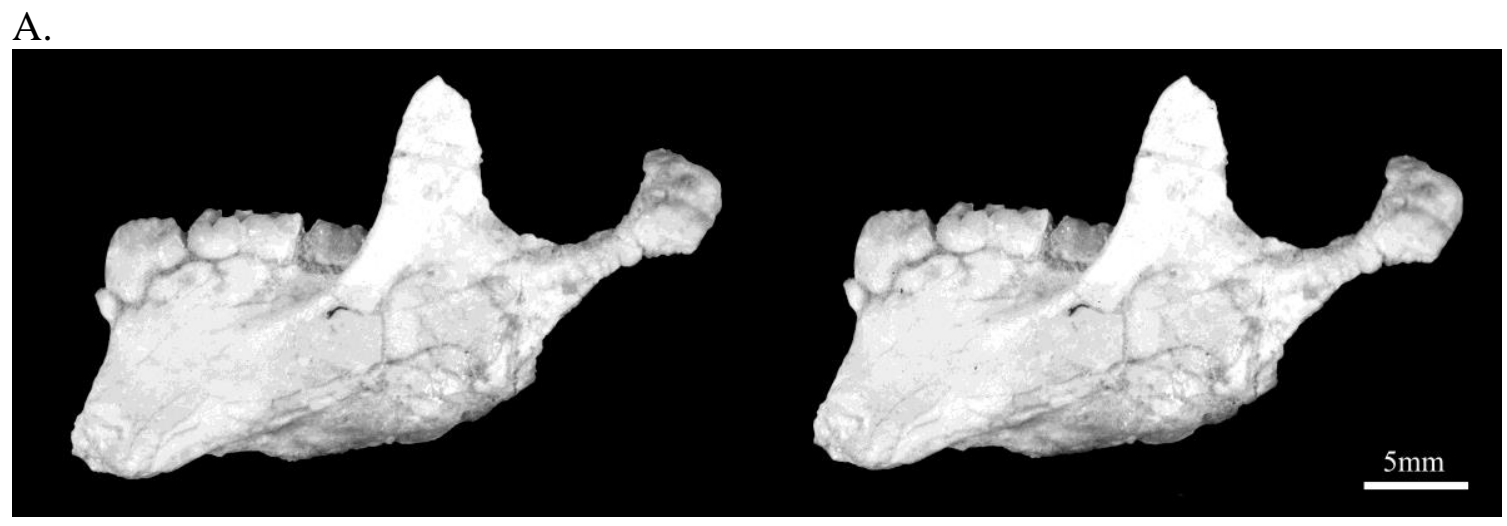

B.

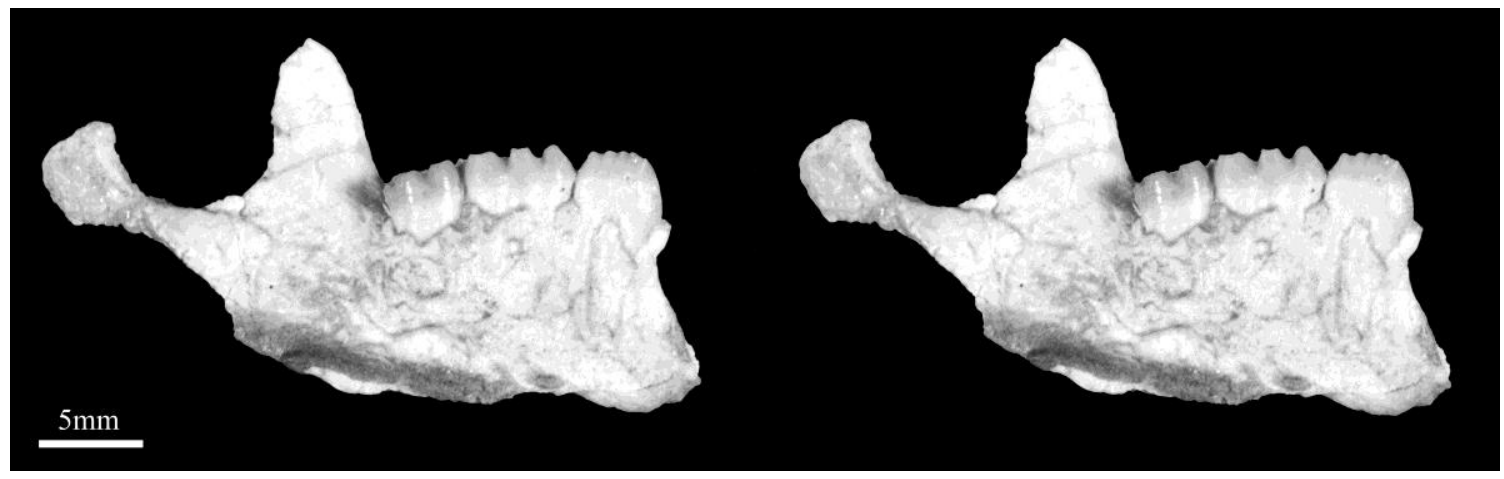

Fig. 12. Stereophotographs of the dentary of PSS-MAE 141, holotype of Mangasbaatar udanii, gen. et sp. nov., in (A) buccal and (B) mesial views. 


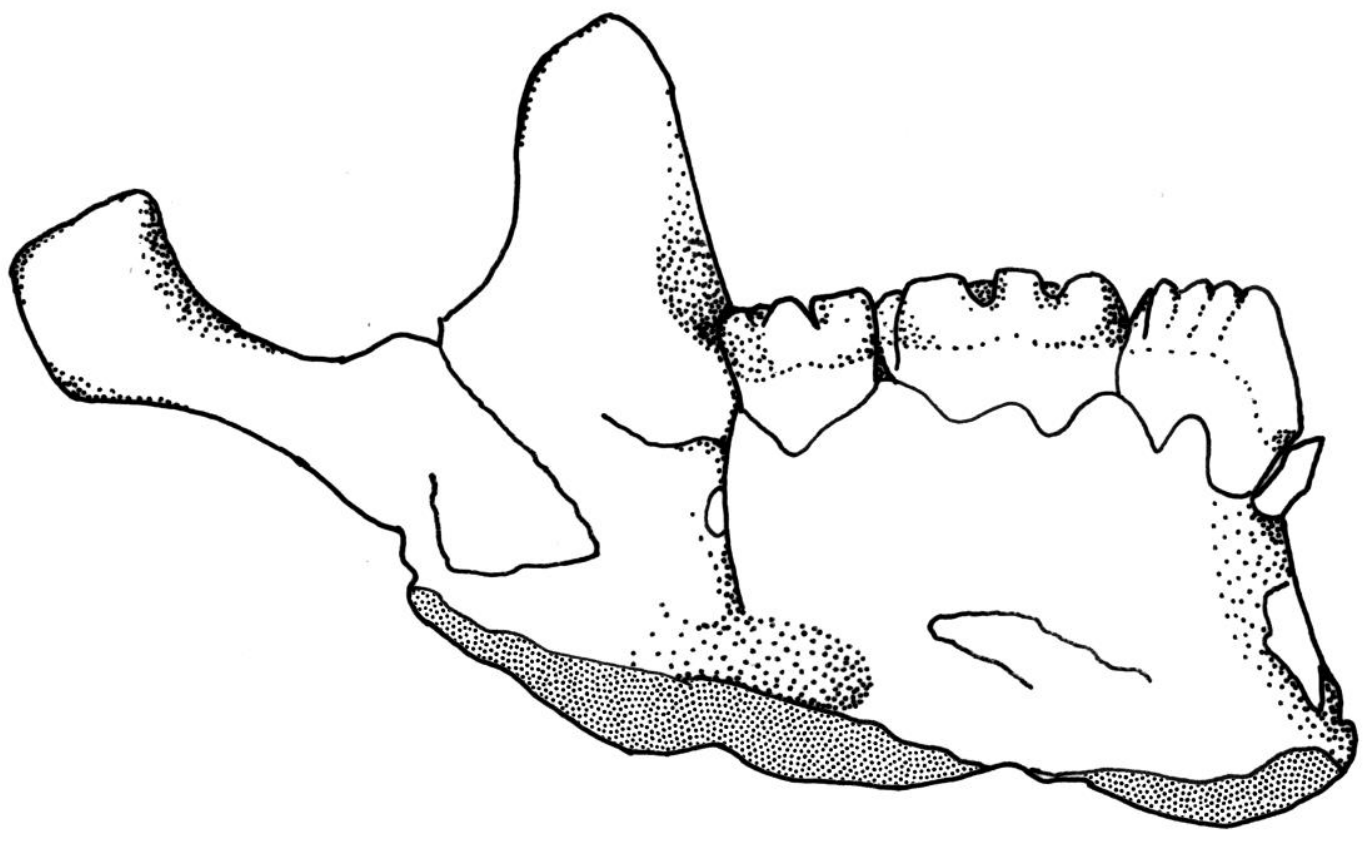

Fig. 13. Illustration of the dentary of PSS-MAE 141 in mesial view. 


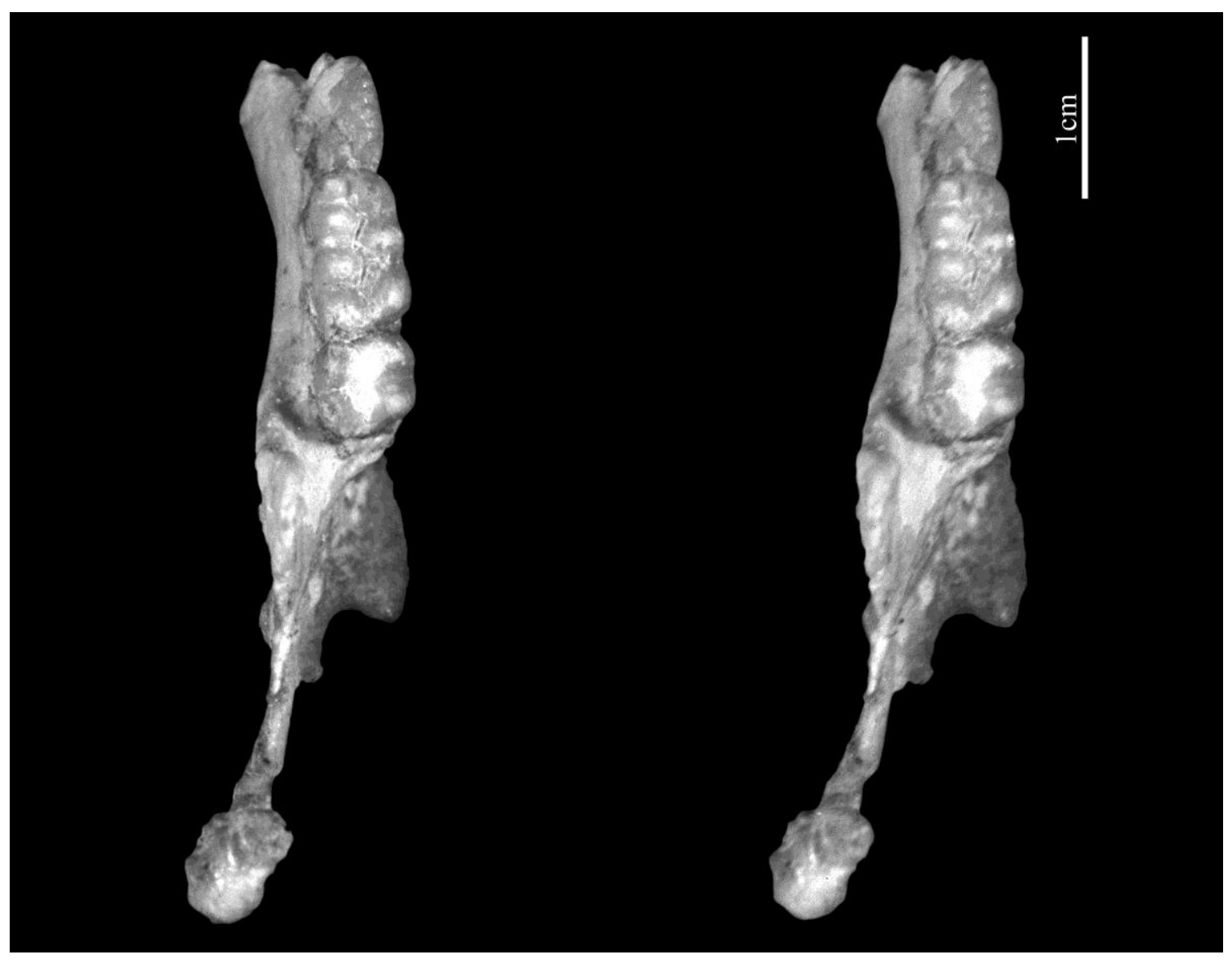

Fig. 14. Stereophotograph of the dentary of PSS-MAE 141, holotype of Mangasbaatar udanii, gen. et sp. nov., in occlusal view. 
A.

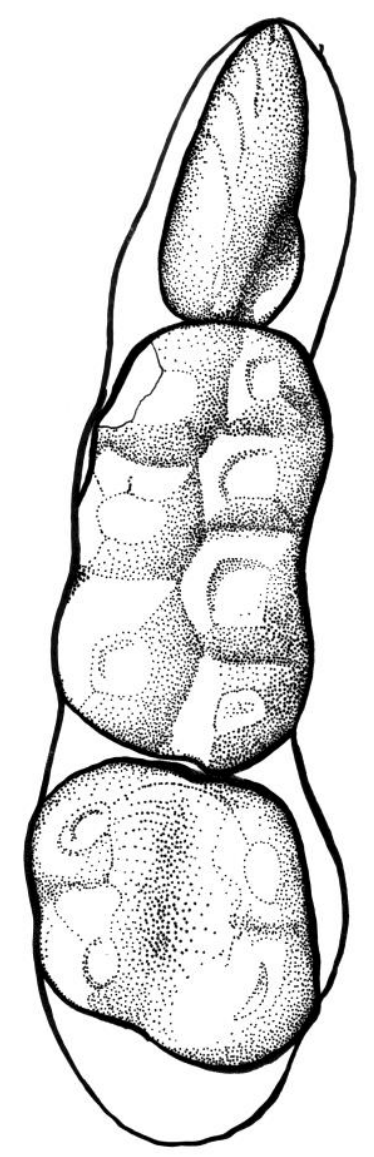

B.

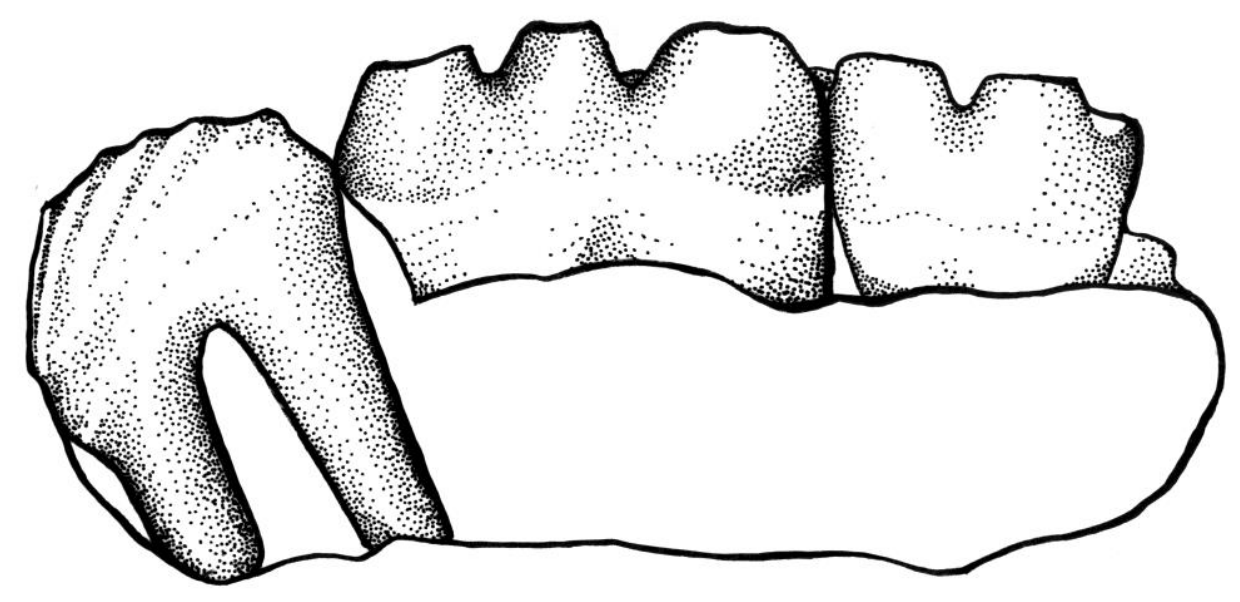

Fig. 15. Illustrations of the lower dention of PSS-MAE 141, holotype of Mangasbaatar udanii, gen. et sp. nov., in (A) occlusal view and (B) fragment of the left dentary of PSSMAE 141 in lateral view. 

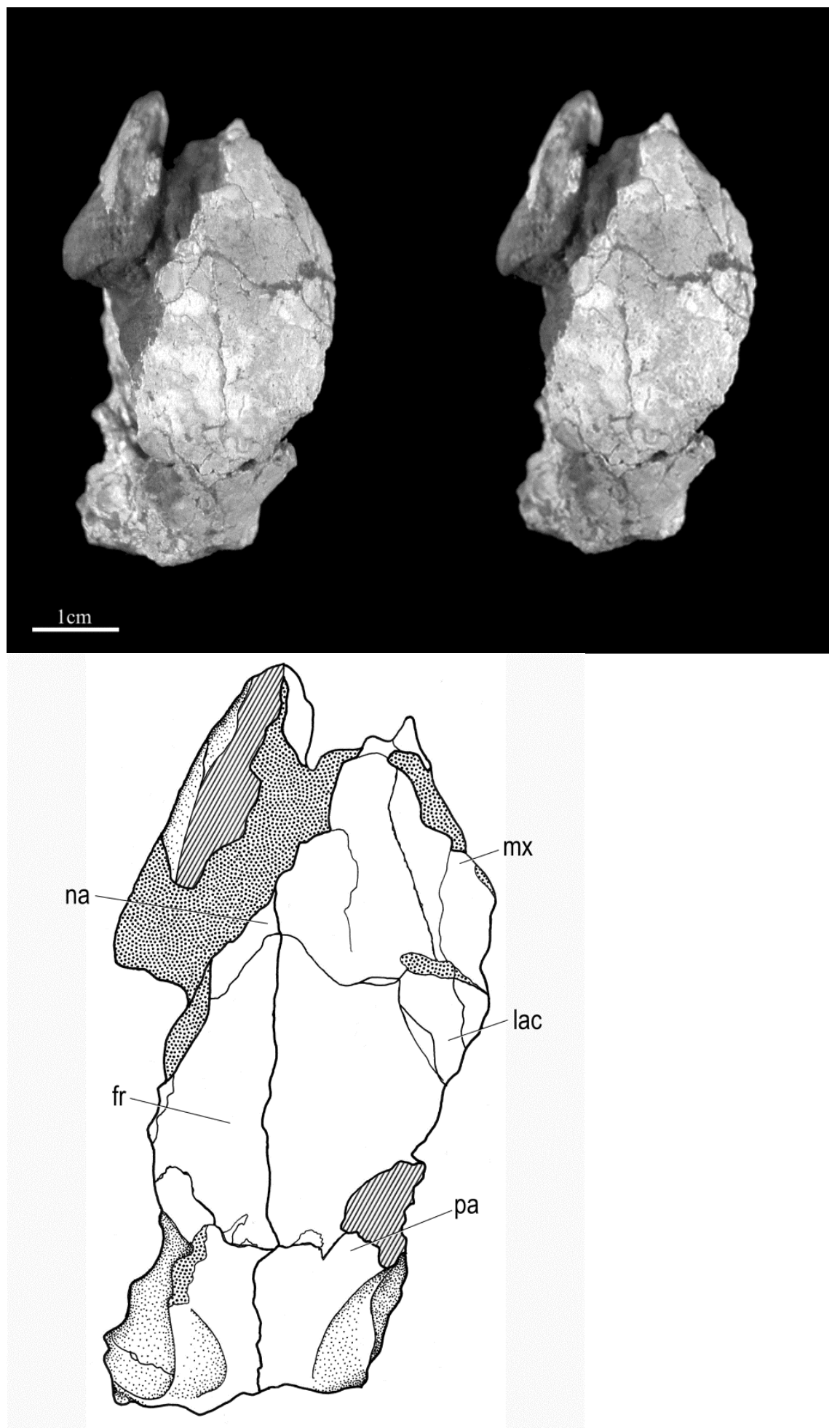

Fig. 16. Stereophotograph of the skull of PSS-MAE 142, Mangasbaatar udanii, gen. et sp. nov., in dorsal view with accompanying line drawing. Dotted pattern represents matrix; parallel lines represent damaged surfaces. Abbreviations: fr frontal; lac lacrimal; mx maxilla; na nasal; pa parietal. 


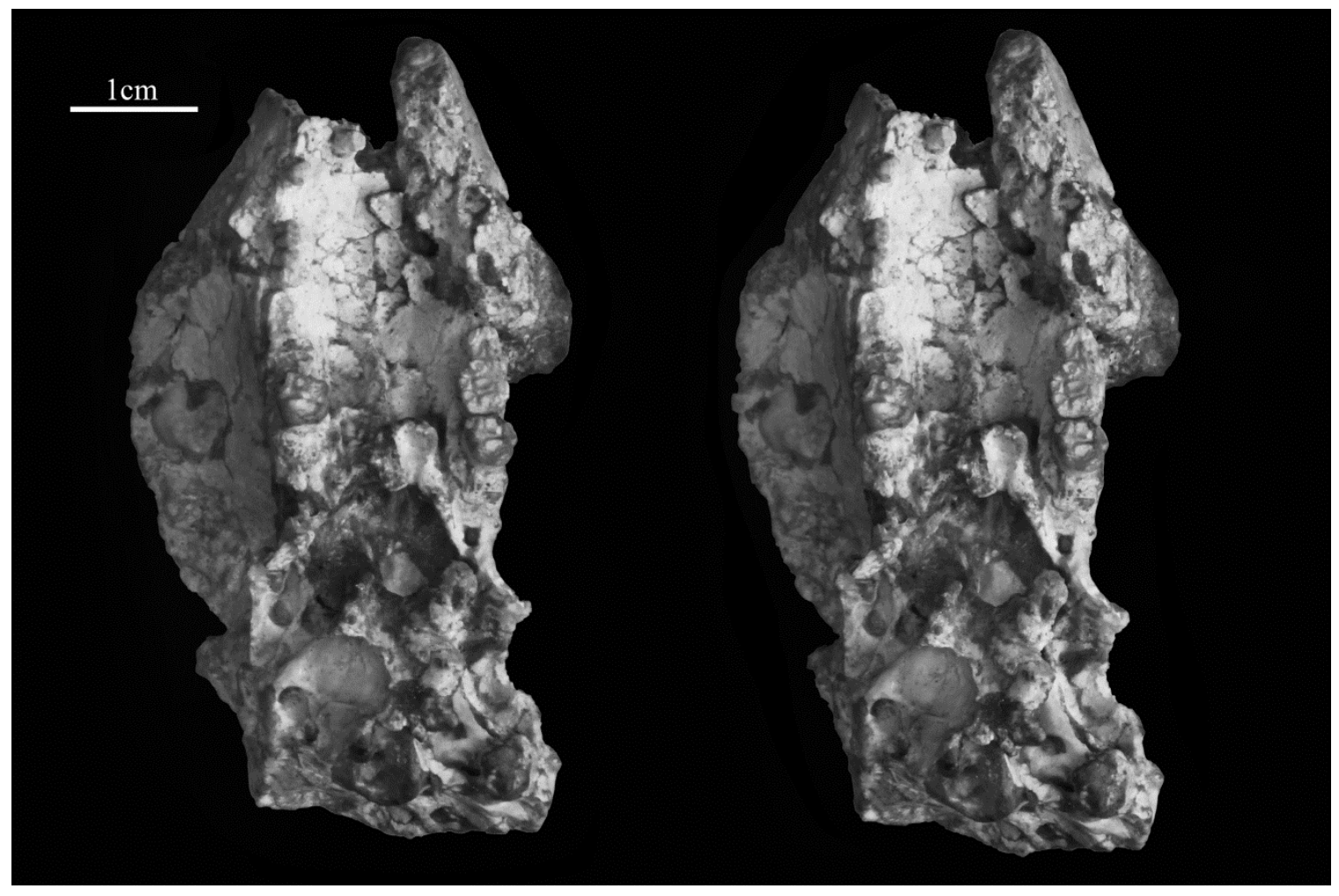

Fig. 17. Stereophotograph of the skull of PSS-MAE 142, Mangasbaatar udanii, gen. et sp. nov., in ventral view. 


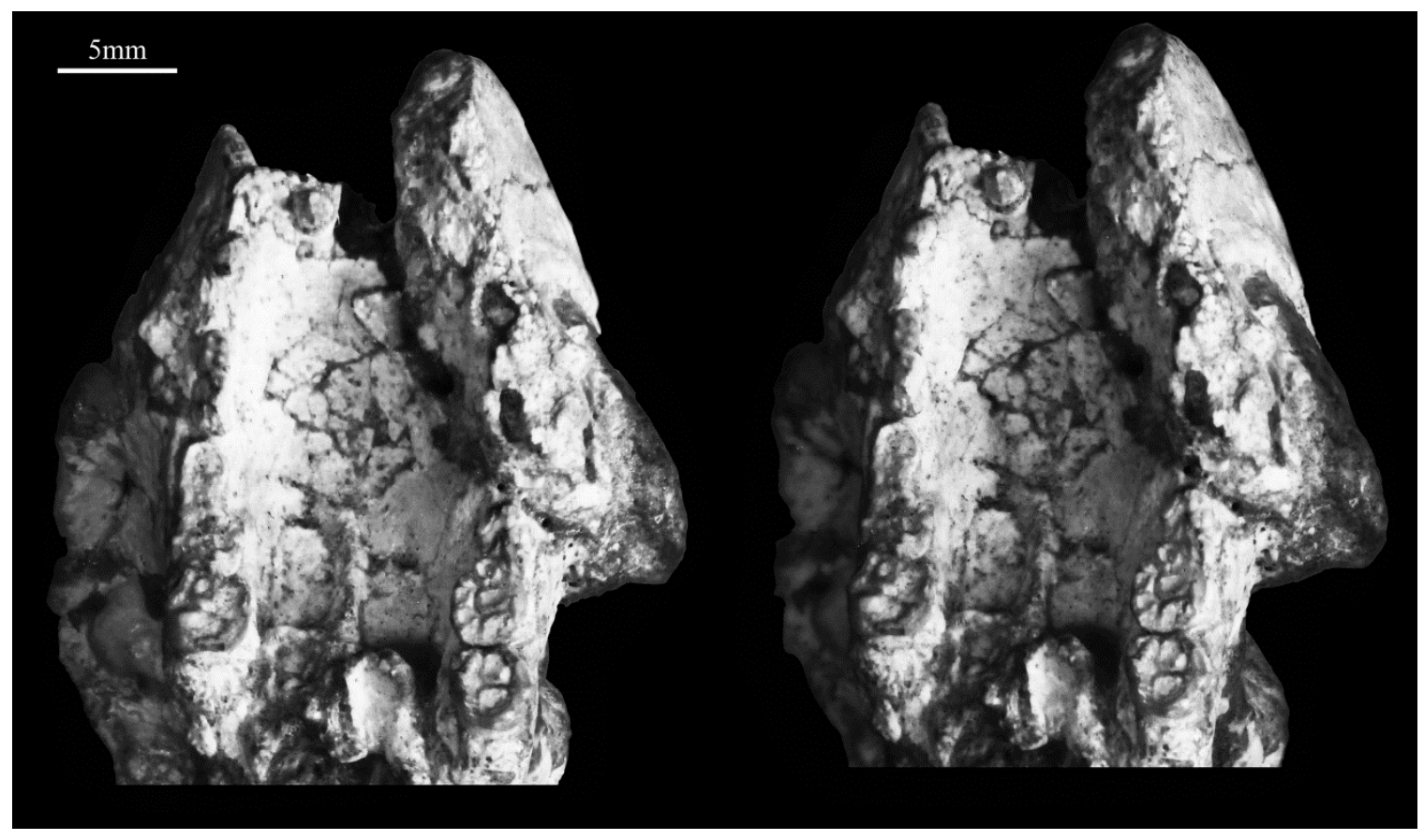

Fig. 18. Stereograph of the palatal region of PSS-MAE 142, Mangasbaatar udanii, gen. et sp. nov., in ventral view. 
A.

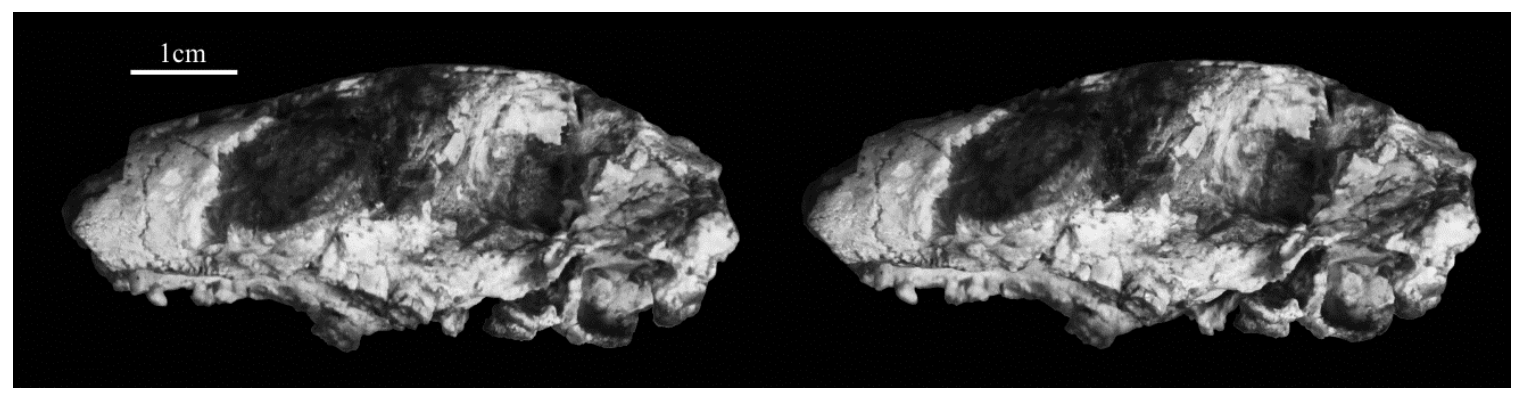

B.

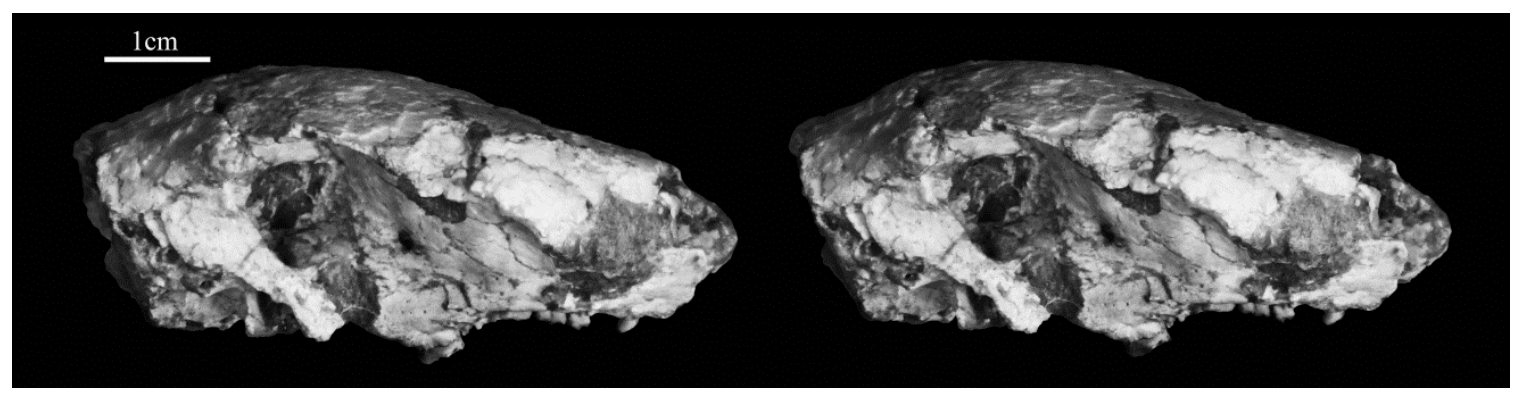

Fig. 19. Stereophotographs of the skull of PSS-MAE 142, Mangasbaatar udanii, gen. et sp. nov., in lateral view from the (A) left and (B) right sides. 


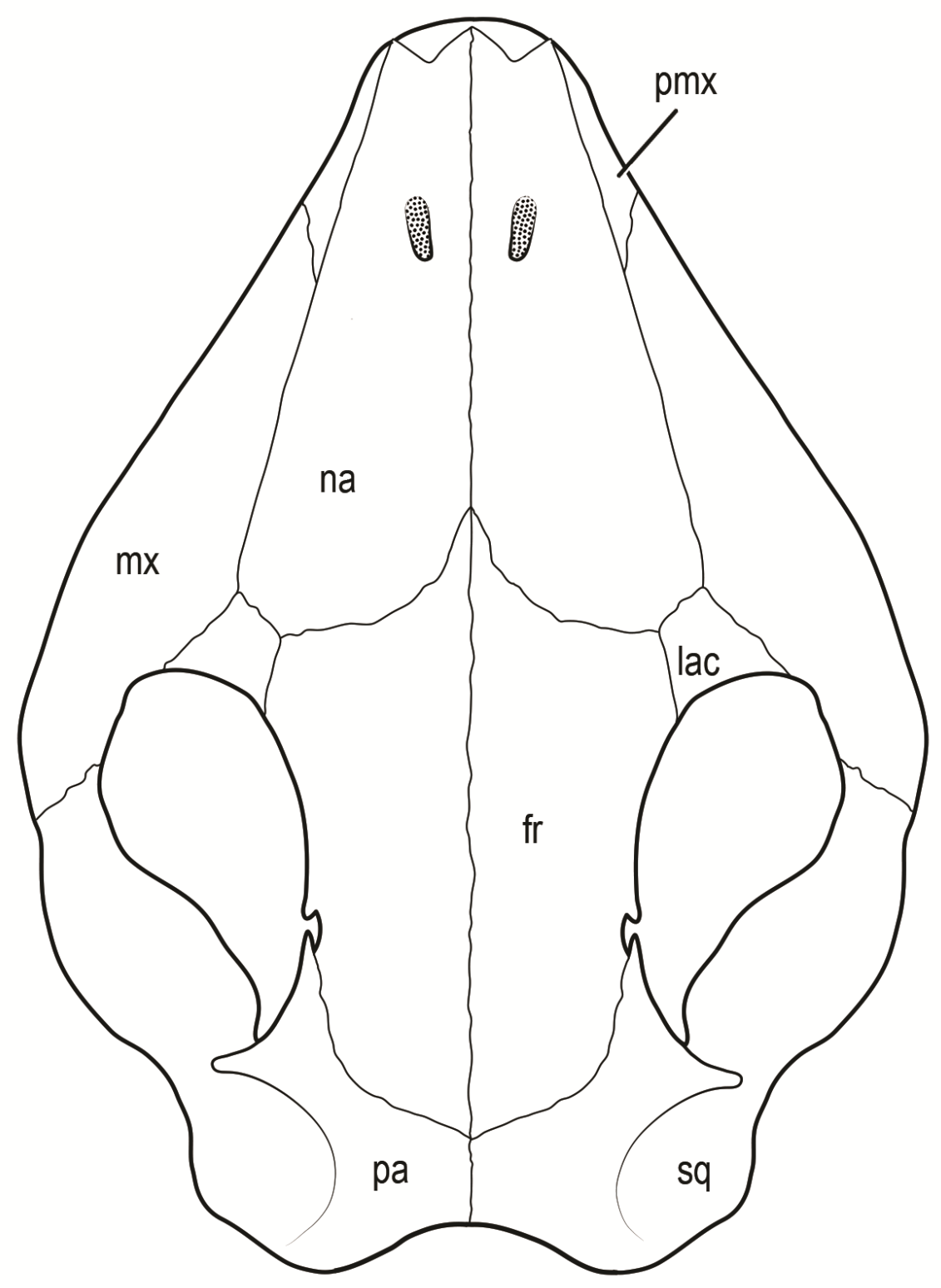

Fig. 20. Reconstruction of the skull of Mangasbaatar in dorsal view. Abbreviations: fr frontal; lac lacrimal; mx maxilla; na nasal; pa parietal; pmx premaxilla; sq squamosal. 


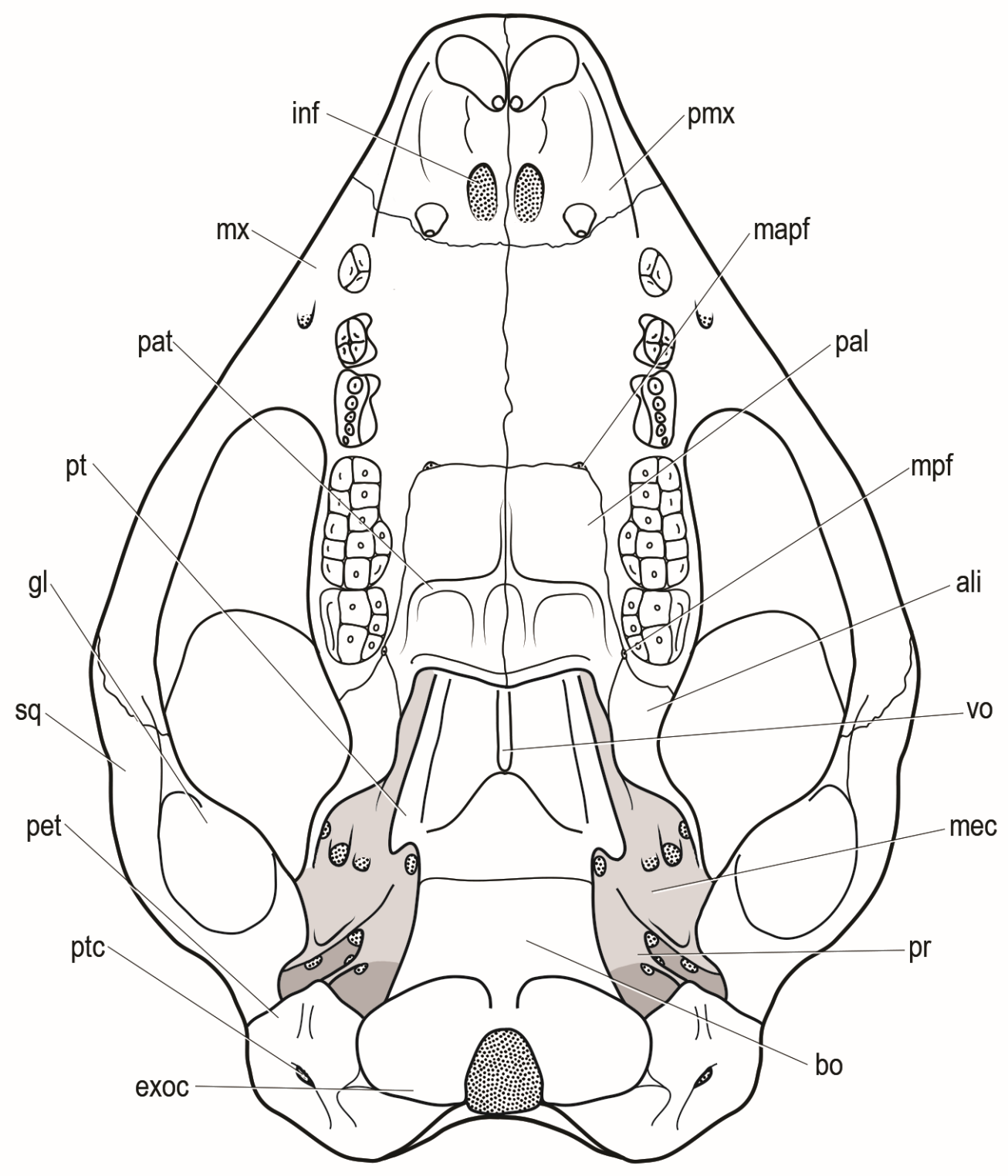

Fig. 21. Reconstruction of the skull of Mangasbaatar in ventral view. Abbreviations: ali alisphenoid; bo basioccipital; exoc exoccipital; gl glenoid; inf incisive foramen; mapf major palatine foramen; mec middle ear cavity; $\mathbf{m p f}$ minor palatine foramen; $\mathbf{m x}$ maxilla; pal palatine; pat postpalatine torus; pet petrosal; pmx premaxilla; pr promontorium; pt pterygoid; ptc posttemporal canal; vo vomer. 


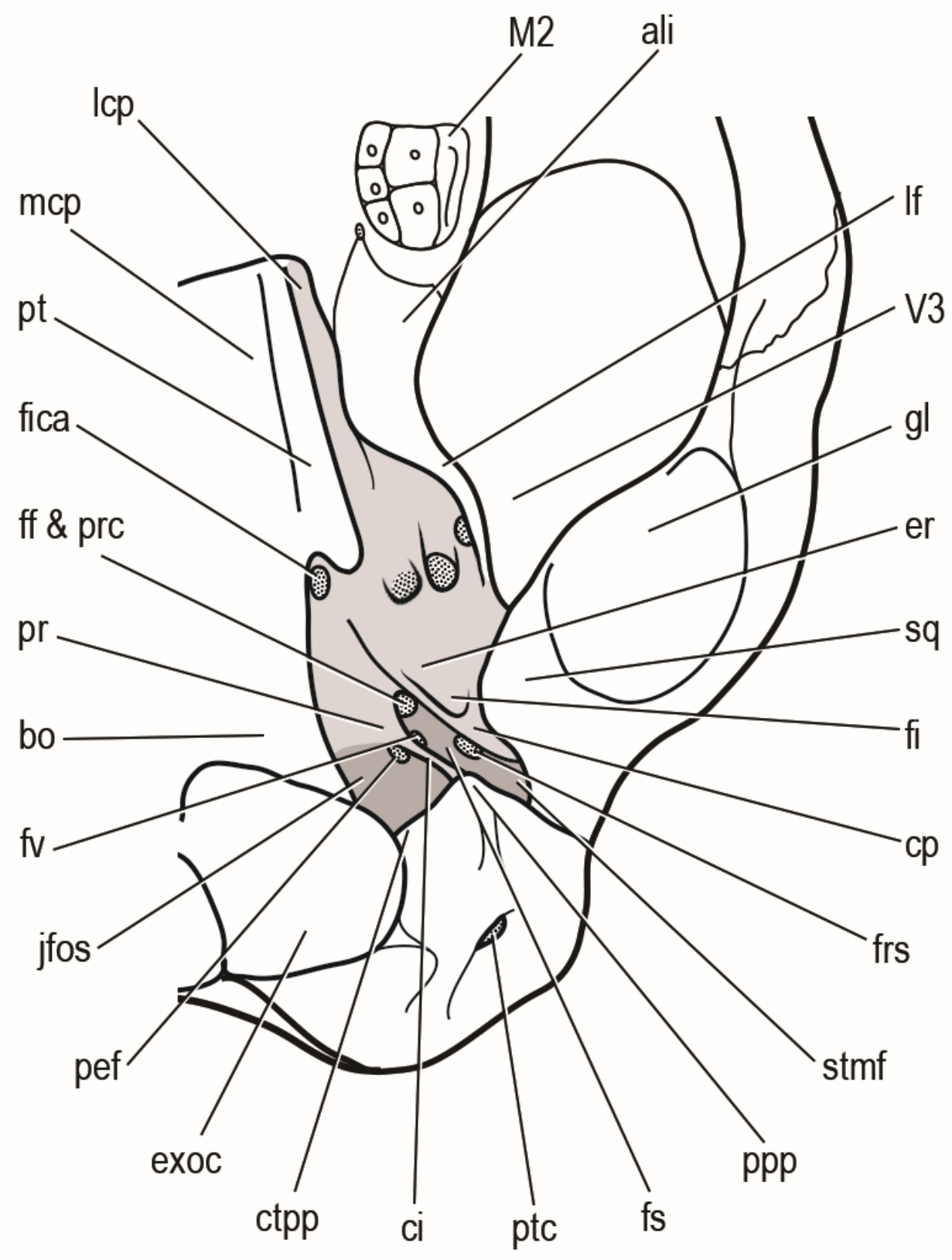

Fig. 22. Reconstruction of the basicranium of Mangasbaatar in ventral view.

Abbreviations: ali alisphenoid; bo basioccipital; ci crista interfenestralis; $\mathbf{c p}$ crista parotica; er epitympanic recess; exoc exoccipital; ff facial foramen; fi fossa incudis; fica foramen for internal carotid artery; frs foramen for ramus superior; fs facial sulcus; fv fenestra vestibuli; gl glenoid; jfos jugular fossa; Icp lateral choanal passage; If lateral flange; M2 upper molar 2; mcp medial choanal passage; pef perilymphatic foramen; ppp pterygoparaoccipital process; pr promontorium; prc prootic canal; pt pterygoid; ptc posttemporal canal; sq squamosal; stmf stylomastoid foramen; $\mathbf{V}_{\mathbf{3}}$ foramina for branches of $v_{3}$ cranial nerve. 


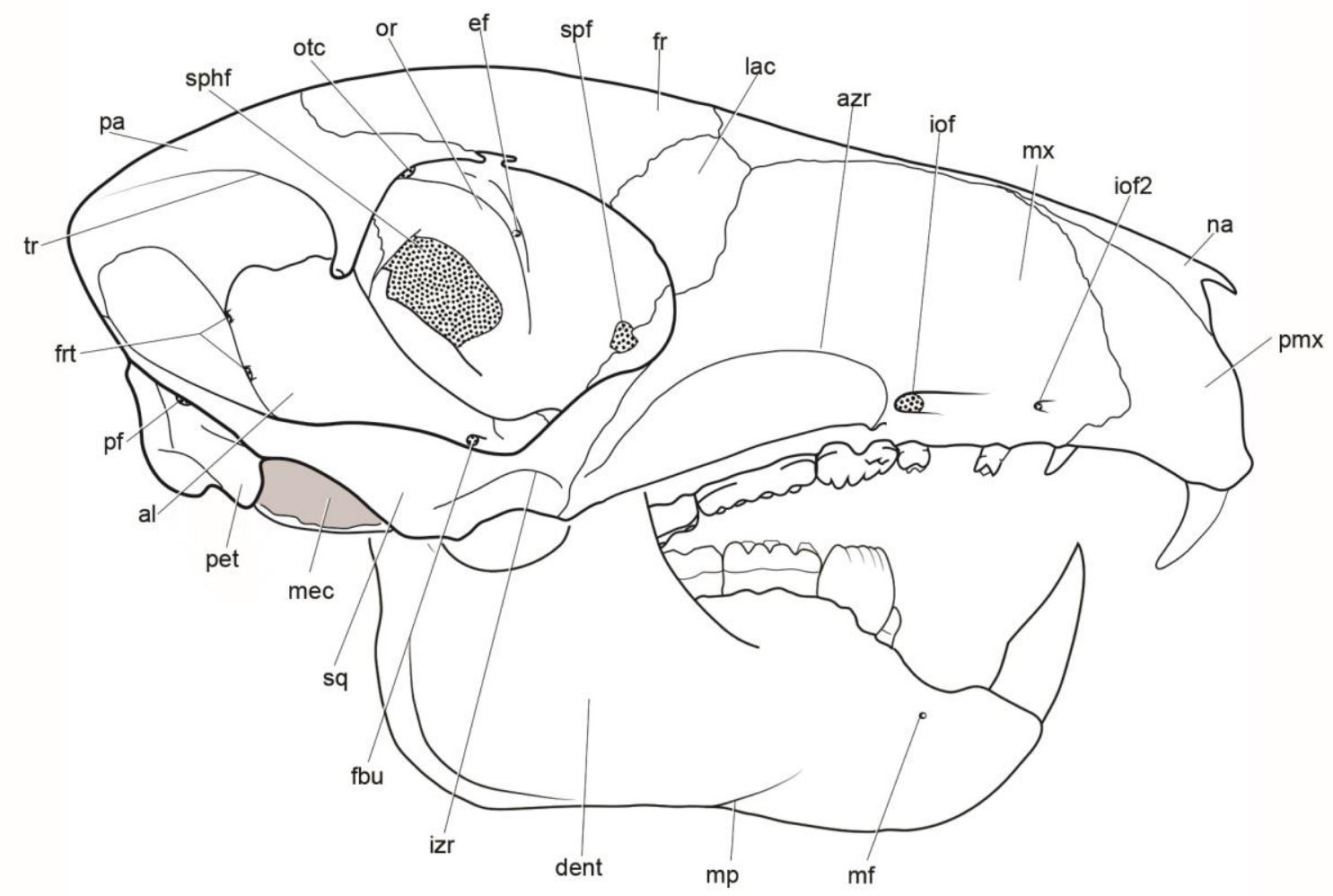

Fig. 23. Reconstruction of the skull and mandible of Mangasbaatar in lateral view. The mandible is in occlusion with the skull in expected life position. Abbreviations: al anterior lamina;azr anterior zygomatic ridge; dent dentary; ef ethmoidal foramen; exoc exoccipital; fbu foramen bucinatorium; fr frontal; frt foramen for ramus temporalis; iof infraorbital foramen; iof2 secondary infrorbital foramen; izr intermediate zygomatic ridge; lac lacrimal; man mandible; mec middle ear cavity; $\mathbf{m f}$ mental foramen; $\mathbf{m p}$ masseteric protuberance; $\mathbf{m x}$ maxilla; na nasal; or orbitosphenoid; otc orbitotemporal canal; pa parietal; pf posttemporal foramen; pet petrosal; pmx premaxilla; spf sphenopalatine foramen; sphf sphenorbital fissure; sq squamosal; tr temporal ridge. 


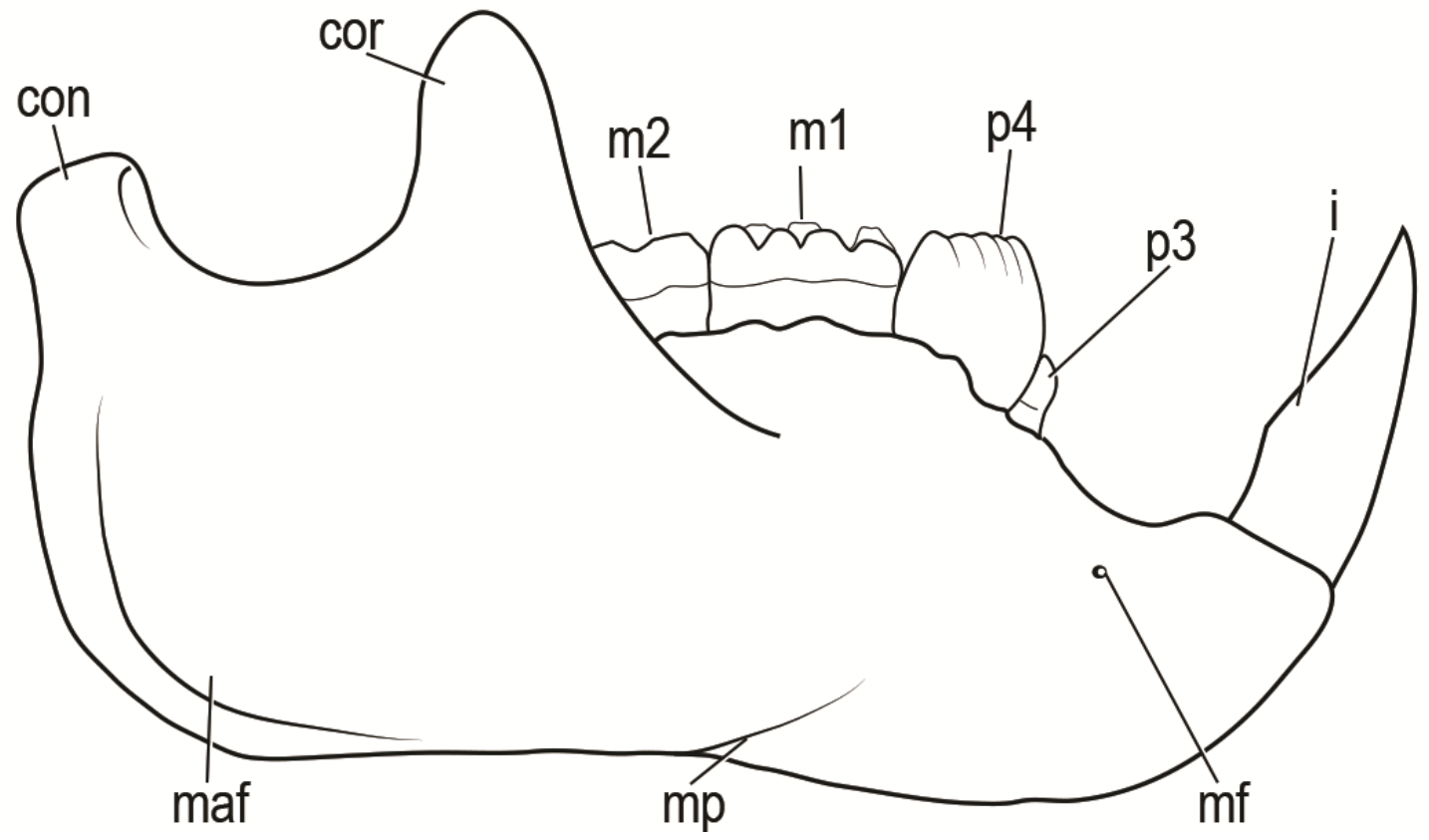

Fig. 24. Reconstruction of the jaw of Mangasbaatar. Abbreviations: con (mandibular) condyle; cor coronoid process; i lower incisor; $\mathbf{m} \mathbf{1}$ first lower molar; $\mathbf{m} \mathbf{2}$ second lower molar; maf masseteric fossa; $\mathbf{m f}$ mental foramen; $\mathbf{m p}$ masseteric protuberance; $\mathbf{p} \mathbf{3}$ third lower premolar; p4 fourth lower premolar. 


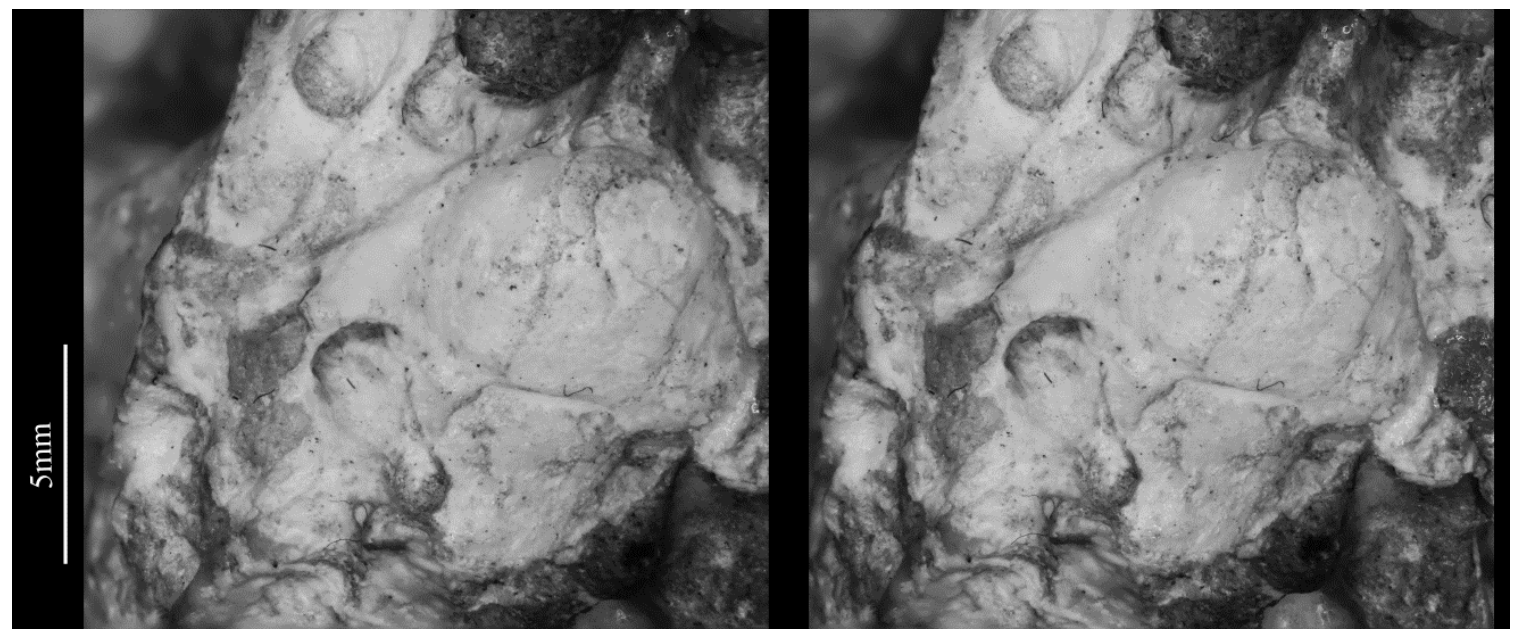

Fig. 25. Stereophotograph of the middle ear region of the right side of PSS-MAE 142, Mangasbaatar udanii, gen. et sp. nov., in ventral view.

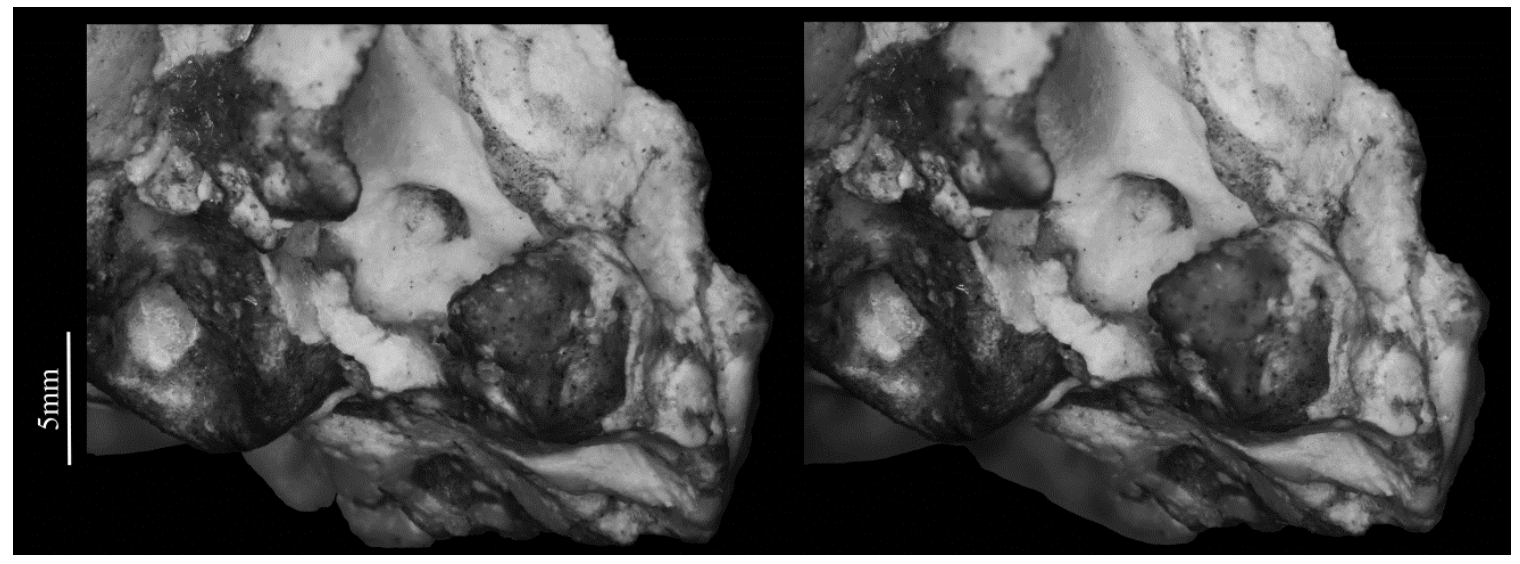

Fig. 26. Stereophotograph of the middle ear region of the left side of PSS-MAE 142, holotype of Mangasbaatar udanii, gen. et sp. nov., in ventral view. 


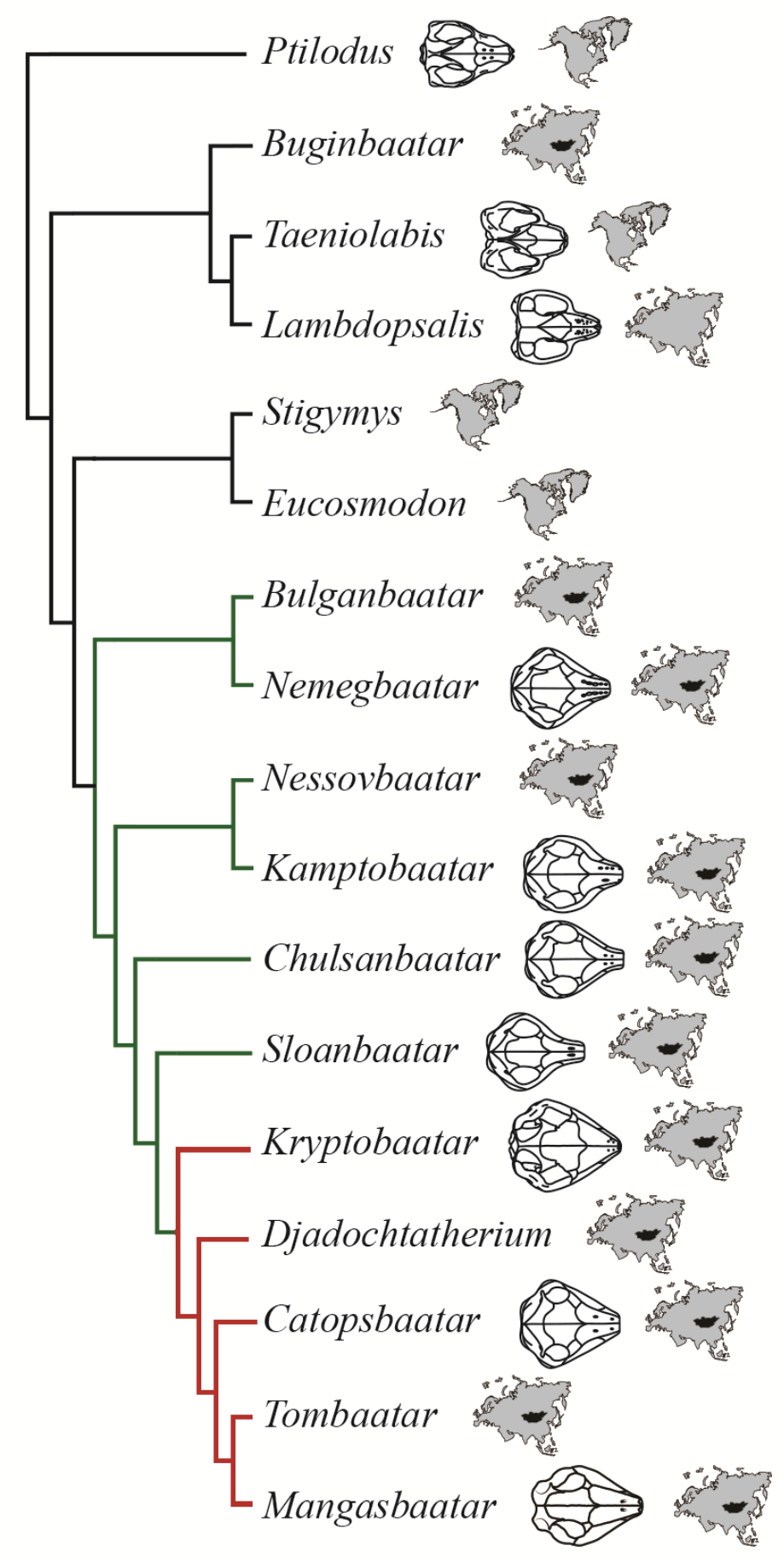

Fig. 27. Phylogenetic tree showing interrelationships between multituberculates. Green branches indicate Djadochtatherioidea. Red branches indicated Djadochtatheriidae. 


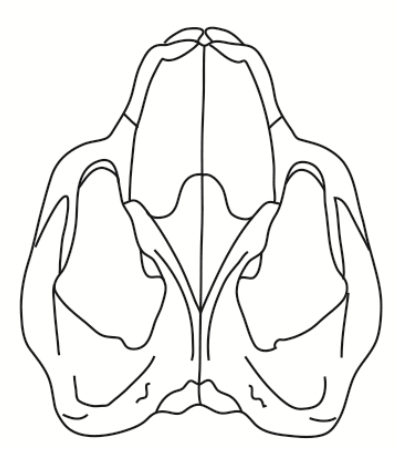

Taeniolabis

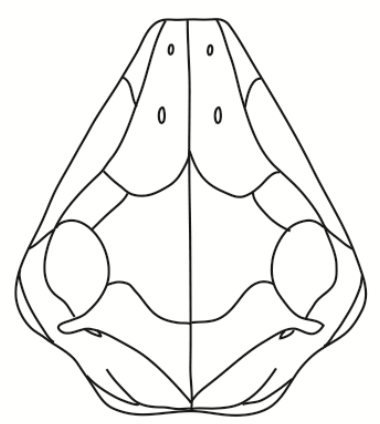

Catopsbaatar

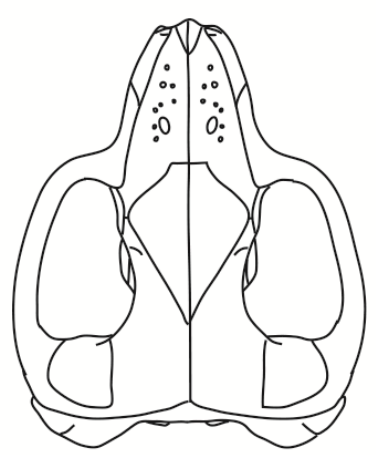

Lambdopsalis

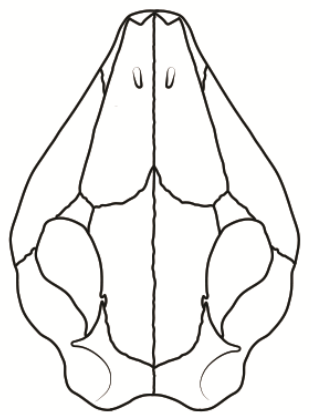

Mangasbaatar

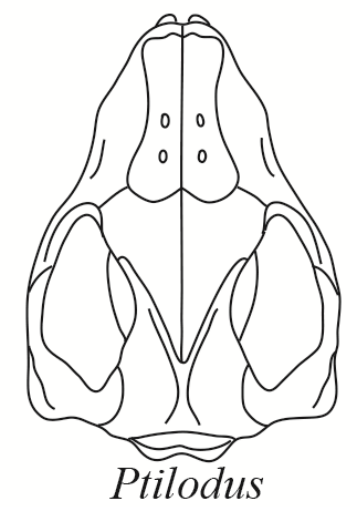

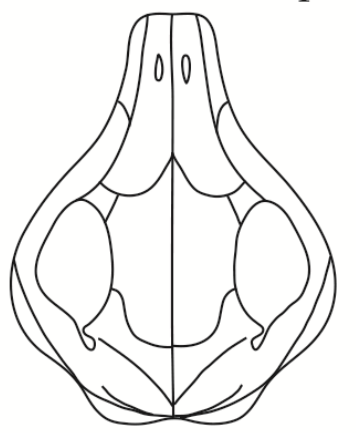

Sloanbaatar

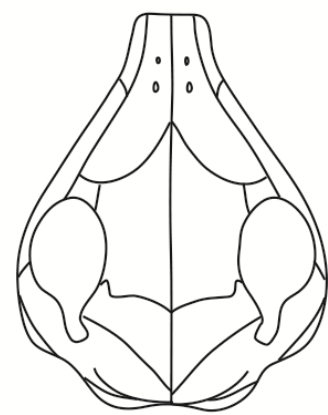

Chulsanbaatar

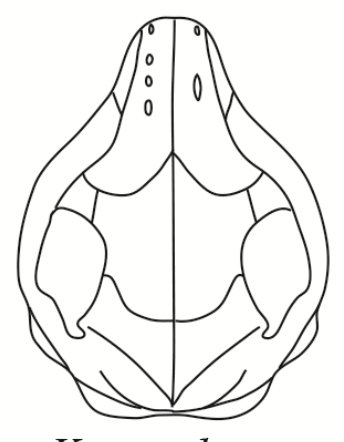

Kamptobaatar

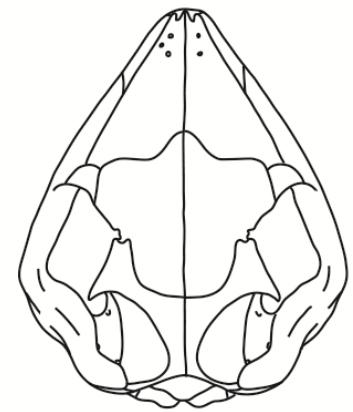

Kryptobaatar

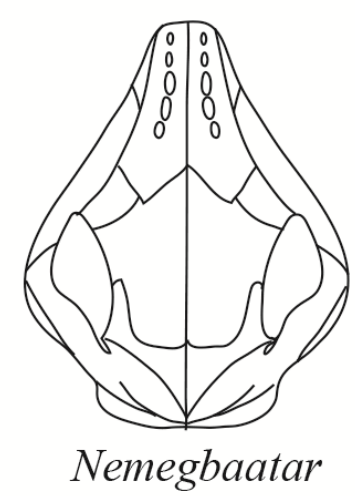

Fig. 28. Collection of multituberculate skull reconstuctions in dorsal view, rendered to be approximately the same length. Drawing is modified from Kielan-Jaworowska et al. (2004) to include Mangasbaatar. 


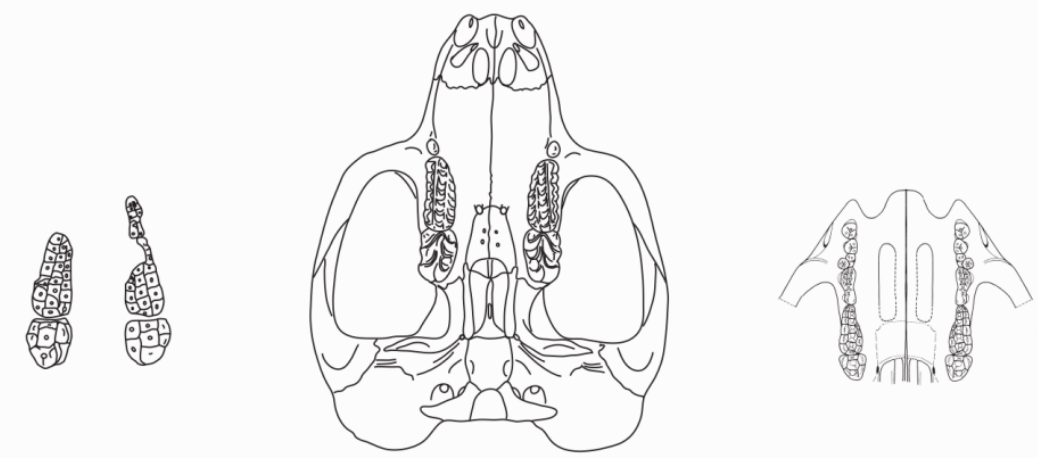

Buginbaatar

Lambdopsalis

Bulganbaatar

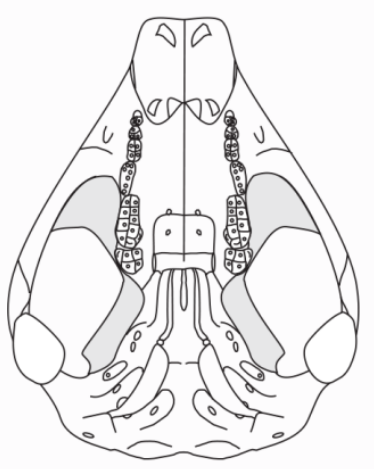

Chulsanbaatar

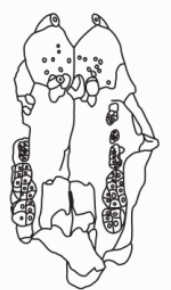

Tombaatar

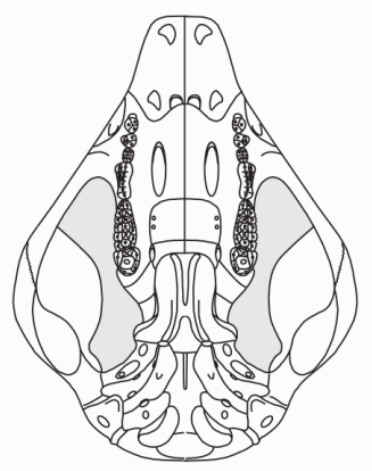

Nemegbaatar

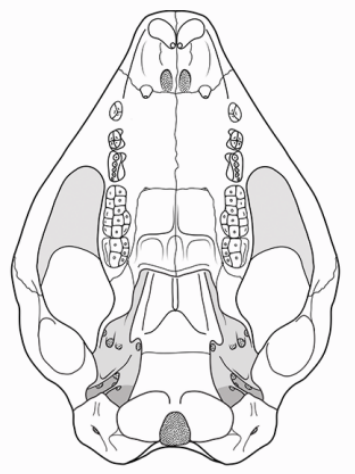

Mangasbaatar

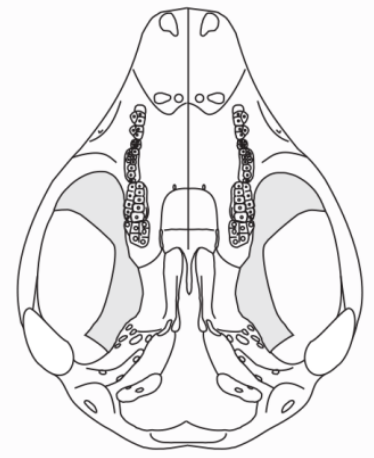

Kamptobaatar

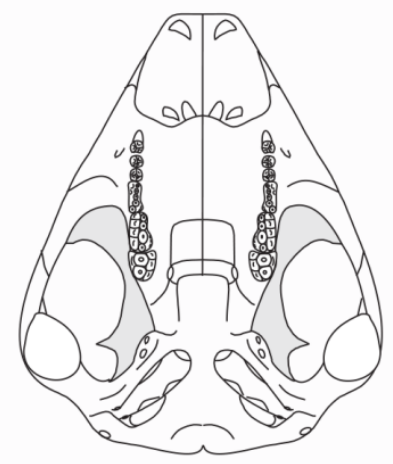

Kryptobaatar

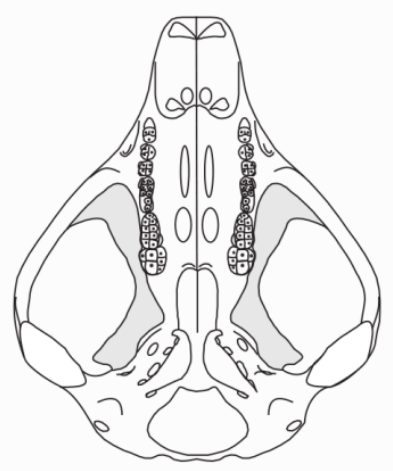

Sloanbaatar

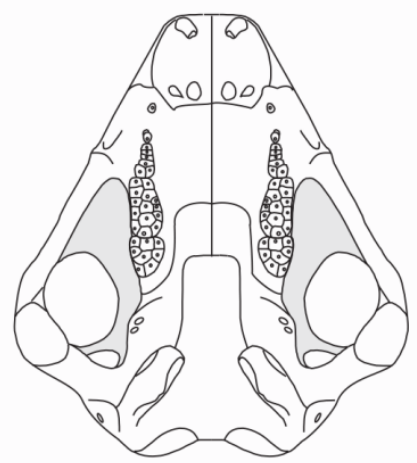

Catopsbaatar

Fig. 29. Collection of multituberculate skull reconstuctions in ventral view, rendered to be approximately the same length. Drawing is modified from Kielan-Jaworowska et al. (2004) to include Mangasbaatar. 


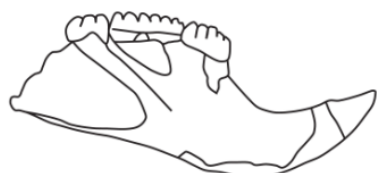

Buginbaatar

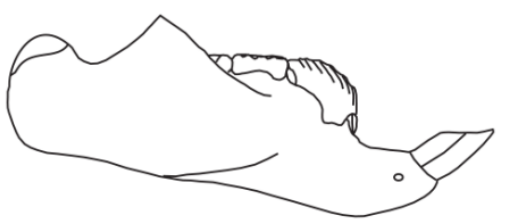

Nemegbaatar

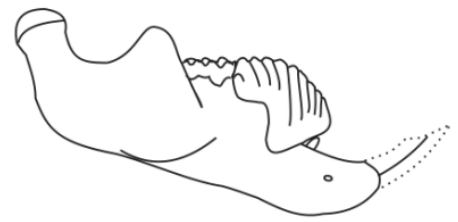

Nessovbaatar

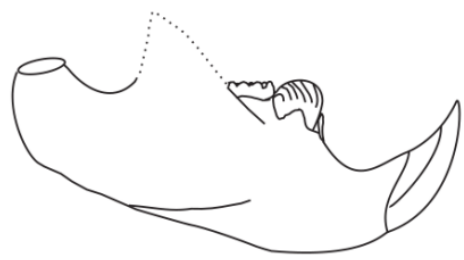

Djadochtatherium

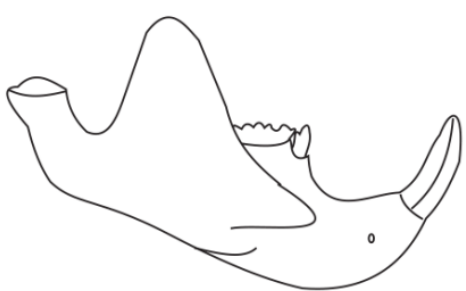

Lambdopsalis

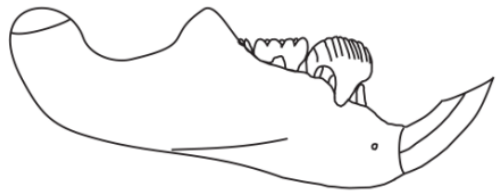

Kamptobaatar

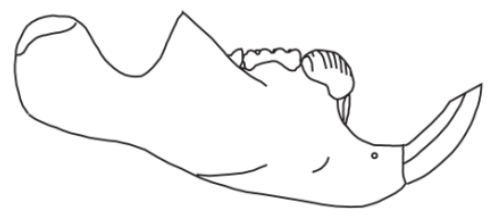

Chulsanbaatar

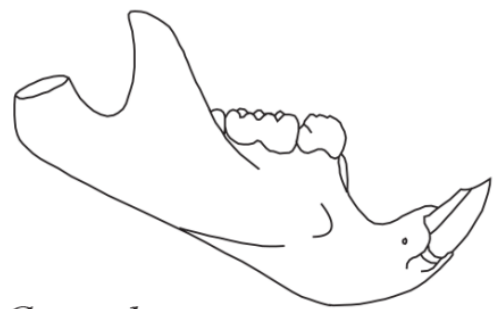

Catopsbaatar

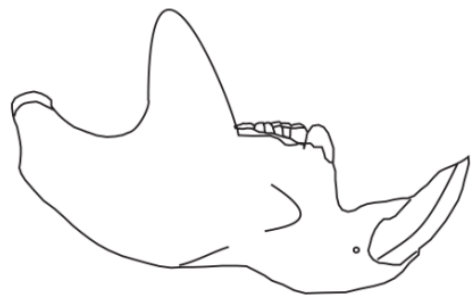

Taeniolabis

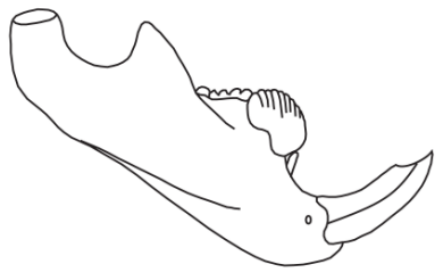

Sloanbaatar

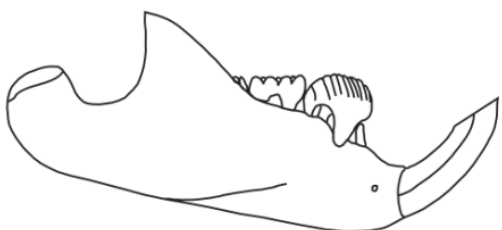

Kryptobaatar

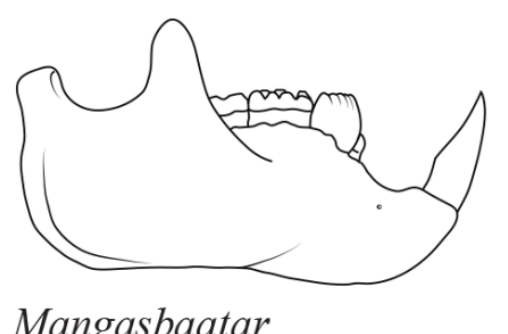

Fig. 30. Collection of multituberculate mandible reconstructions in lateral view, rendered to be approximately the same length. Drawing is modified from KielanJaworowska et al. (2004) to include Mangasbaatar. 


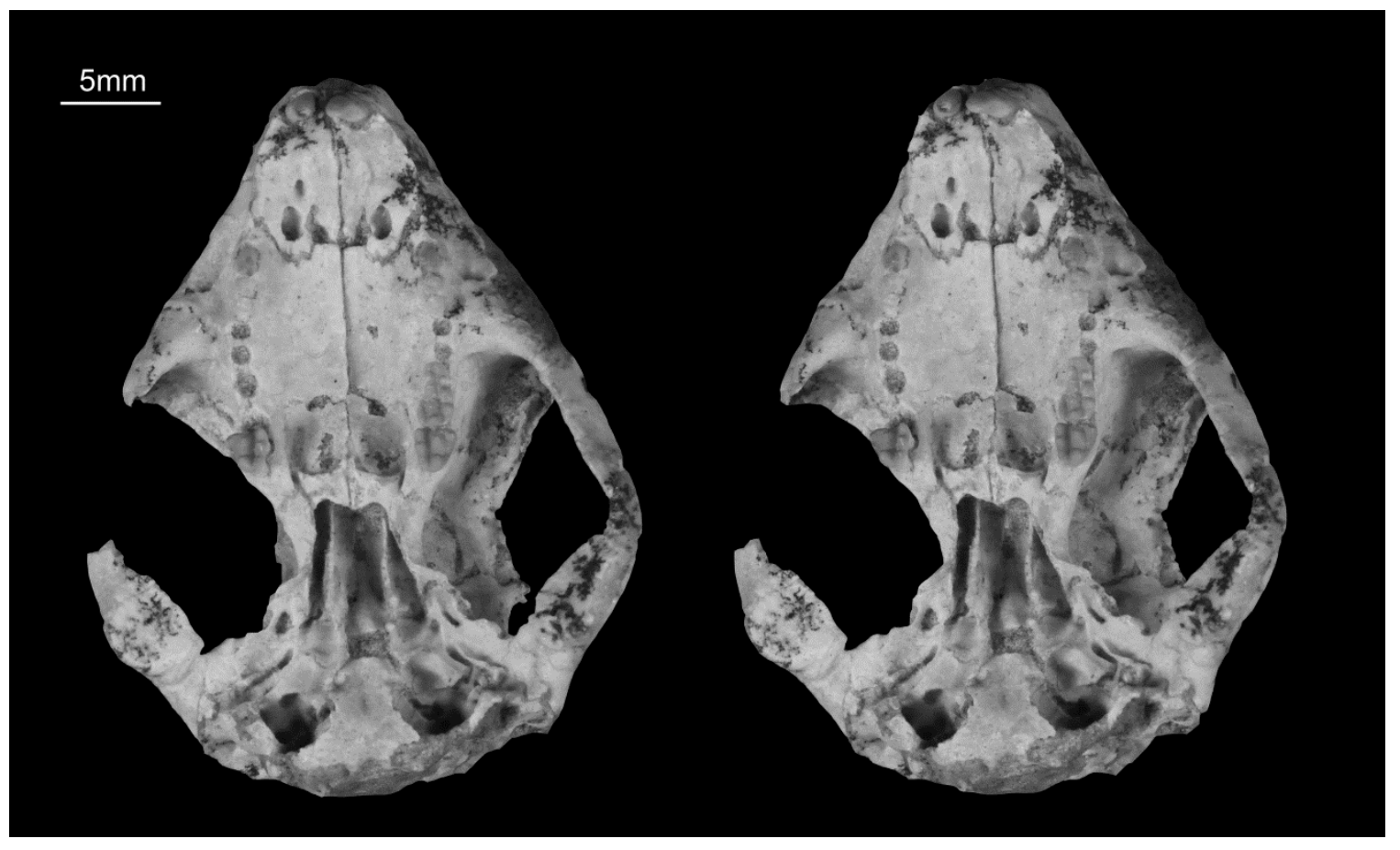

Fig. 31. Stereophotograph of the skull of an unpublished specimen of Kryptobaatar, in ventral view, field number PSS-MAE 93-191, from the Late Cretaceous of Mongolia. 


\section{REFERENCES}

Andres, B. and Norell, M.A. 2005. The first record of a pterosaur from the Lower Cretaceous sediments of Öösh. American Museum Novitates 3472: 1-6.

Ashwell, K. (Ed). 2013. Neurobiology of Monotremes: brain evolution in our distant mammalian cousins. CSIRO PUBLISHING. Collingwood, Australia.

Barghusen, H. R. 1986. On the evolutionary origin of the therian tensor veli palatini and tensor tympani muscles. In N. Hutton III, P. O. MacLean, J. J. Roth, and E. C. Roth (eds.), Ecology and Biology of Mammal-like Reptiles: 253-262. Washington: Smithsonian Institution Press.

Chiappe, L. M., Norell, M. A., Clark, J. M. 1998. The skull of a relative of the stem-group bird Mononykus. Nature 392: 275-278.

Dashzeveg, D., Novacek, M. J., Norell, M. A., Clark, J.M., Chiappe, L. M., Davidson, A. R., McKenna, M. C., Dingus, L., Swisher III, C. C., and Altangerel, P. 1995. Extraordinary preservation in a new vertebrate assemblage from the Late Cretaceous of Mongolia. Nature 374: 446-449.

Dashzeveg, D., Dingus, L., Loope, D. B., Swisher III, C. C., Dulam, T., and Sweeney, M. R. 2005. New stratigraphic subdivision, depositional environment and age estimate for the Upper Cretaceous Djadochta Formation, Southern Ulan Nur Basin, Mongolia. American Museum Novitates 3498:31pp. 
Dingus, L., Loope, D. B., Dashzeveg, D., Swisher III, C. C., Minjin, C., Novacek, M. J., Norell, M. A. 2008. The Geology of Ukhaa Tolgod (Djadokhta Formation, Upper Cretaceous, Nemegt Basin, Mongolia). American Museum Novitates 3616: 40pp.

Dong, Z-M., Currie, P. J. 1993. Protoceratopsian embryos from Inner Mongolia, People's republic of China. Canadian Journal of Earth sciences 30: 22482254.

Evans, H. E., and Christensen, G. C. 1979. Anatomy of the Dog. Philadelphia:

W. B. Saunders.

Fleischer, G. 1978. Evolutionary principles of the mammalian middle ear. Advances in Anatomy, Embryology and Cell Biology 55: 1-70.

Freeman, E. F.

1976. Mammal teeth from the forest marble (Middle Jurassic) of Oxfordshire, England. Science 194: 1053-1055.

1979. A Middle Jurassic mammal bed from Oxfordshire. Palaeontology 22: 135-166.

Gambaryan, P. P., and Kielan-Jaworowska, Z. 1995. Masticatory musculature of Asian taeniolabidoid multituberculate mammals. Acta Palaeontologica Polonica 40(1): 45-108.

Gao, K., and Hou, L. 1996. Systematics and taxonomic diversity of squamates from the Upper Cretaceous Djadochta Formation, Bayan Mandahu, Gobi Desert, People's Republic of China. Canadian Journal of Earth Sciences 33 (4): 578-598.

Gao, K., and Norell, M. A.

1998. Taxonomic revision of Carusia (Reptilia: Squamata) from the Late Cretaceous of the Gobi Desert and phylogenetic relationships of anguimorphan lizards. American Museum Novitates 3250: 1-51.

2000. Taxonomic composition, and systematics of Late Cretaceous lizard assemblages from Ukhaa Tolgod and adjacent localities, Mongolian Gobi Desert. Bulletin of the American Museum of Natural History 249: 1-118. 
Gidley, J. W. 1909. Notes on the fossil mammalian genus Ptilodus with descriptions of new species. Proceedings U.S. National Museum 36(1689): 611-627.

Gradzinski, R., Kielan-Jaworowska, Z., and Maryanska, T. 1977. Upper Cretaceous Djadokhta, Barun Goyot and Nemegt formations of Mongolia, including remarks on previous subdivisions. Acta Geologica Polonica 27: 281-318.

Gregory, W. K., and Simpson, G. G. 1926. Cretaceous mammal skulls from Mongolia. American Museum Novitates 225: 20 pp.

Griffiths, M.1978. The Biology of the Monotremes. London: Academic Press. Hahn, G.

1985. Zum bau des infraorbital-foramens bei den Paulchoffatiidae (Multituberculata, Ober-Jura). Berliner geowiss 60: 5-27.

1987. Neue beobachtungen zum schadel-und gebiss-bau der Paulchoffatiidae (Multituberculata, Ober-Jura). Palaeovertebrata 17: 155196.

Hahn, G., and Hahn, R.1994. Nachweis des septomaxillare bei Pseudcobolodon krebsin. sp. (Multituberculata) aus dem Malm Portugals. Berliner geowiss 13: 9-29.

Heffner, R. S., Koay, G., Heffner, H. E. 2001. Audiograms of five species of rodents: implications for the evolution of hearing and the perception of pitch. Hearing research 157: 138-152.

Hill, J. E. 1935. The cranial foramina in rodents. Journal of Mammology 16: 121129.

Hinchcliffe, R., Pye, A. 1969. Variations in the middle ear of the Mammalia. J. Zool. 157, 277-288.

Hopson, J. A., Kielan-Jaworowska, Z., Allin, E. F. 1989. The cryptic jugal of multituberculates. Journal of Vertebrate Paleontology 9: 201-209.

Hopson, J. A., and Rougier, G. W. 1993. Braincase structure in the oldest known skull of a therian mammal: implications for mammalian systematics and cranial evolution. American Journal of Science 293: 268-299. 
Horovitz, I. 2000. The tarsus of Ukhaatherium nessovi (Eutharia, Mammalia) from the Late Cretaceous of Mongolia: an appraisal of the evolution of the ankle in basal therians. Journal of Vertebrate Paleontology 20: 547-560.

Hurum, J. H.

1994. Snout and orbit of Cretaceous Asian multituberculates studied by serial sections. Acta Palaeontologica Polonica 39: 181-221.

1998a. The inner ear of two Late Cretaceous multituberculate mammals, and its implications for multituberculate hearing. Journal of Mammalian Evolution 5: 65-93.

1998b. The braincase of two Late Cretaceous Asian multituberculates studied by serial directions. Acta Paleontologica Polonica 43: 21-52.

Hurum, J. H., Presley, R., and Kielan-Jaworowska, Z. 1995.Multituberculate ear ossicles. In A.-L. Sun and Y. Wang (eds.), Sixth Symposium on Mesozoic Terrestrial Ecosystems and Biota, Short Papers, 243-246. China Ocean Press, Beijing.

Hurum, J. H., Presley, R., and Kielan-Jaworowska, Z. 1996. The middle ear in multituberculate mammals. Acta Palaeontologica Polonica 41: 253-275.

Jenkins, F. A., Gatesy, S. M., Shubin, N. H., and Amaral, W. W. 1997. Haramyids and Triassic mammalian evolution. Nature 385: 715-718.

Jerzykiewicz, T., Koster, E. H., and Zheng, J.-J. 1993. Djadokhta Formation correlative strata in Chinese Inner Mongolia: an overview of the stratigraphy, sedimentary geology, and paleontology and comparisons with the type locality in the pre-Altai Gobi. Geologial Survey of Canada 39793: 2180-2195.

Jerzykiewicz, T., and Russell, D. A. 1991. Late Mesozoic stratigraphy and vertebrates of the Gobi Basin. Cretaceous Research 12: 345-377.

Jerzykiewicz, T. 2000. Lithostratigraphy and sedimentary settings of the Cretaceous dinosaur beds of Mongolia. In M. J. Benton, M. A. Shishkin, D. M. Unwin, and E. N. Kurochkin (eds), the age of dinosaurs in Russia and Mongolia: 279-296. Cambridge: Cambdridge University Press.

Jerzykiewicz, T., Currie, P. J., Eberth, D. A., Johnston, P. A., Koster, E. H., and Zheng, J.-J. 1993. Djadokhta Formation correlative strata in Chinese Inner Mongolia: an overview of stratigraphy, sedimentary geology, and 
paleontology and comparison with type locality in pre-Altaian Gobi. Canadian Journal of Earth Sciences 30: 2180-2195.

Joyce, W. G. and Norell, M. A. 2005. Zangarlia ukhaachelys, new species, a nanhsiungchelyid turtle from the Late Cretaceous of Ukhaa Tolgod, Mongolia. American Museum Novitates 3481: 1-19.

Kemp, T. S. 1983. The relationships of mammals. Zoological Journal of the Linnean Society 77: 353-384.

Kermack, K. A., and Kielan-Jaworowska, Z. 1971. Therian and non-therian mammals. Zoological Journal of the Linnean Society 50(suppl. 1): 103115.

Kermack, K. A., Mussett, F., and Rigney, H.W. 1981. The skull of Morganucodon. Zoological Journal of the Linnean Society 71: 1-158.

Kielan-Jaworowska, Z.

1969a. Discovery of a multituberculate marsupial bone. Nature 222: 10911092.

1969b. Preliminary data on the Upper Cretaceous eutherian mammals from Bayn Dzak, Gobi Desert. Results of the Polish-Mongolian Palaeontological Expeditions. Part I. Acta Palaeontologia Polonica 19: 171-191.

1970. New Upper Cretaceous multituberculate genera from Bayn Dzak, Gobi Desert. Results of the Polish-Mongolian Paleontological Expedition. Part II. Acta Palaeontologica Polonica 21: 35-54.

1971. Results of the Polish-Mongolian palaeontological expeditions - part III. Skull structure and affinities of the multituberculata. Acta Palaeontologica Polonica 5: 5-41.

1974. Multituberculate succession in the Late Cretaceous of the Gobi Desert (Mongolia). Plates V-XXI. Results of the Polish-Mongolian Palaeontological Expedition. Part V. Acta Palaeontologia Polonica 30: 2344.

1975a. Evolution of the therian mammals in the Late Cretaceous of Asia. Part I. Deltatheridiidae. Results of the Polish-Mongolian Palaeontological Expeditions. Part VI. Acta Palaeontologia Polonica 33: 103-132. 
1975b. Possible occurrence of marsupial bones in Cretaceous eutherian mammals. Nature 255: 598-599.

1975c. Preliminary description of two new eutherians genera from the Late Cretaceous of Mongolia. Acta Palaeontologica Polonica 33: 5-16.

1977. Evolution of the therian mammals in the Late Cretaceous of Asia. Part II. Postcranial skeleton in Kennalestes and Asioryctes. Results of the Polish-Mongolian Palaeontological Expeditions. Part VII. Acta Palaeontologia Polonica 37: 65-83.

1978. Evolution of the therian mammals in the Late Cretaceous of Asia. Part III. Postcranial skeleton in Zalambdalestidae. Acta Palaeontologia Polonica 38: 5-41.

1979. Evolution of the therian mammals in the Late Cretaceous of Asia. Part III. Postcranial skeleton in Zalambdalestidae. Results of the PolishMongolian Expeditions. Part VIII. Acta Palaeontologica Polonica 38: 3-41.

1984a. Evolution of the therian mammals in the Late Cretaceous of Asia. Part V. Skull structure in Zalambdalestidae. Results of the PolishMongolian Expeditions. Part X. Acta Palaeontologica Polonica 46: 107117.

1984b. Evolution of the therian mammals in the Late Cretaceous of Asia. Part VII. Synopsis. Results of the Polish-Mongolian Expeditions. Part XII. Acta Palaeontologica Polonica 46: 173-183.

1994. A new generic name for the multituberculate mammal "Djadochtatherium" catopsaloides. Acta Palaeontologica Polonica 39: 134-136.

2013. In pursuit of early mammals. Bloomington, Indiana: Indiana University Press. ISBN 978-0-253-00817-6.

Kielan-Jaworowska, Z. and Dashzeveg, D. 1978. New Late Cretaceous mammal locality in Mongolia and a description of a new multituberculate. Acta Palaeontologica Polonica 23: 115-130.

Kielan-Jaworowska, Z. and Gambaryan, P. P. 1994. Postcranial anatomy and habits of Asian multituberculate mammals. Fossils \& Strata 36: 1-92.

Kielan-Jaworowska, Z. and Hurum, J. H.

1997. Djadochtatheria - a new suborder of multituberculate mammals. Acta Palaeontologica Polonica 42: 201-242. 
2001. Phylogeny and Systematics of Multituberculate Mammals.

Palaeontology 44: 389-429.

2005. Postcranial skeleton of a Cretaceous multituberculate mammal Catopsbaatar. Acta Paleontologica Polonica 53 (4): 545-566.

Kielan-Jaworowska, Z., Hurum, J. H., Currie, P. M., and Barsbold, R. 2002. New data on anatomy of the Late Cretaceous multituberculate mammal Catopsbaatar. Acta Paleontologica Polonica 47: 557-560.

Kielan-Jaworowska, Z., Hurum, J. H., and Lopatin, A. V. 2005. Skull structure in Catopsbaatar and the zygomatic ridges in multituberculate mammals. Acta Paleontologica Polonica 50 (3): 487-512.

Kielan-Jaworowska, Z., Presley, R., and Poplin, C. 1986. The cranial vascular system in taeniolabidoid multituberculate mammals. Philosophical Transactions of the Royal Society of London 313: 525-602.

Kielan-Jaworowska, Z., Hurum, J. H., and Badamgarav, D. 2003. Multituberculate mammal Kryptobaatar and the distribution of mammals in the Upper Cretaceous rocks of the Gobi Desert. Acta Palaeontologica Polonica 48: 161-166.

Kielan-Jaworowska, Z., Cifelli, R. \& Luo, Z.-X. 2004. Mammals from the Age of Dinosaurs: Origins, Evolution, and Structure. Columbia University Press.

Kielan-Jaworowska, Z., and Sochava, A. V. 1969. The first multituberculate from the Uppermost Cretaceous of the Gobi Desert (Mongolia). Acta Palaeontologica Polonica 14: 355-367.

Kielan-Jaworowska, Z., and Trofimov, B. A. 1980. Cranial morphology of the Cretaceous eutherians mammal Barunlestes. Acta Palaeontogica Polonica 25: 167-185.

Kik, P. 2002. Computed tomographic (CT) examination of the craniomandibular morphology of Kryptobaatar dashzevegi (Mammalia Multituberculata). Masters dissertation. University of Louisville. 92pp.

Klaauw, C. J. van der. 1931. The auditory bulla in some fossil mammals. Bulletin of the American Museum of Natural History. 62: 1-352.

Ksepka, D. T., and Norell, M. A. 2004. Ornithomimosaur cranial material from Ukhaa Tolgod (Omnogov, Mongolia). American Museum Novitates 3448: $1-4$. 
Kuhn, H.-J. 1971. Die Entwicklung und Morphologie des Schädels von Tachyglossus aculeatus. Abhandlungen der Senckenbergischen Naturforschenden Gesellschaft 528: 1-192.

Kuhn, H.-J., and Zeller, U. 1987. The cavum epiptericum in monotremes and therian mammals. In H.-J. Kuhn and U. Zeller (eds.). Mammalia Depicta, Heft 13, Beihefte zur Zeitschrift für Säugetierekunde, 51-70. Paul Parey, Hamburg/ Berlin.

Kuhn, H.-J., and Zeller, U. (eds.). 1987. Morphogenesis of the Mammalian Skull. Mammalia Depicta 13: 50-70. Hamburg: Paul Parey.

Kurzanov, S. M. 1992. A gigantic protoceratopsid from the Upper Cretaceous of Mongolia. Paleontological Journal 24: 85-91.

Ladevèze S., Asher, R. J., Sánchez-Villagra, M. R. 2008. Petrosal anatomy in the fossil mammal Necrolestes: evidence for metatherian affinities and comparisons with the extant marsupial mole. Journal of Anatomy 213: 686-697.

Lillegraven, J. A., and Krusat, G. 1991. Cranio-mandibular anatomy of Haldanodon exspectatus (Docodonta; Mammalia) from the Late Jurassic of Portugal and its implications to the evolution of mammalian characters. Contributions to Geology, University of Wyoming 28: 39-138.

Loope, D. B., Dingus, L., Swisher, C. C., and Minjin, C. 1998. Life and death in a Late Cretaceous dune field. Geology 26: 27-30.

Makovicky, P. J., Norell, M. A., Clark, J. M., and Rowe, T. 2003. Osteology and relationships of Byronosaurus jaffei (Theropoda: Troodontidae). American Museum Novitates 3403: 1-32.

Makovicky, P. J., and Norell, M. A. 1998. A partial ornithomimid braincase from Ukhaa Tolgod (Upper Cretaceous, Mongolia). American Museum Novitates 3247: 1-16.

Mason, M. J.,

2003. Morphology of the middle ear of golden moles (Chrysochloridae). Journal of Zoology 260: 391-403.

2004. The middle ear apparatus of the tuco-tuco Ctenomys sociabilis (Rodentia, Ctenomyidae). Journal of Mammology 85: 797-805. 
2006. Evolution of the middle ear apparatus in talpid moles. Journal of Morphology 267: 678-695.

2013. Of mice, moles and guinea pigs: Functional morphology of the middle ear in living mammals. Hearing research 301: 4-18.

Matthew, W. D. 1928. The evolution of mammals in the Eocene. Proceedings of the Zoological Society of London 1928: 947-985.

Matthew, W. D.,Granger, W., and Simpson, G. G. 1929. Additions to the fauna of the Gashato Formation of Mongolia. American Museum Novitates 376: 112.

Meng, J. 1992. The stapes of Lambdopsalis bulla (Multituberculata) and transformational analyses on some stapedial features in Mammaliaformes. Journal of Vertebrate Paleontology 12: 459-471.

Minjin, B. 2008. Descriptions of Three New Specimens of Cimilodontans and a Phylogenetic Study of the Postcranial Anatomy of Multituberculata (Mammalia, Synapsida). ProQuest. ISBN 0549507183, 9780549507185

Miao, D. 1988. Skull morphology of Lambdopsalis bulla (Mammalia, Multituberculata) and its Implications to Mammalian Evolution. Contributions to Geology, University of Wyoming (special paper 4): 1-104.

Moore, K. L., and. Agur, A. M. R. 2002. Essentials of Clinical Anatomy, Second Edition. Lippincott, Williams and Wilkins. New York, 691 pp.

de Muizon, C., Cifelli, R. L., Paz, R. C. 1997. The origin of the dog-like borhyaenoid marsupials of South America. Nature 389: 486-489.

Norell, M. A., Clark, J. M., Dashzeveg, D., Rhinchen, B., Chiappe, L. M., Davidson, A. R., McKenna, M.C., Altangerel, P., Novacek, M. J. 1994. A therapod dinosaur embryo and the affinities of the Flaming Cliffs dinosaur eggs. Science 266: 779-782.

Norell, M. A., Makovicky, P. J., and Clark, J. M. 2000. A new troodontid theropod from Ukhaa Tolgod, Mongolia. Journal of Vertebrate Paleontology 20(1): 7-11.

Norell, M. A., Clark, J. M., and Chiappe, L. M. 2001. An embryonic oviraptorid (Dinosauria: Tetrapoda) from the Upper Cretaceous of Mongolia. American Museum Novitates 3315: 1-17. 
Norell, M. A., Clark, J. M., Turner, A. H., Makovicky, P. J., Barsbold, R., and Rowe, T. 2006. A new dromaeosaurid theropod from Ukhaa Tolgod (Ömnögov, Mongolia). American Museum Novitates 3545:1-25.

Norell, M. A., and Hwang, S. H. 2004. A troodontid dinosaur from Ukhaa Tolgod (Late Cretaceous Mongolia). American Museum Novitates 3446: 1-9.

Novacek, M., Norell, M. A., McKenna, M. C. and Clark, J. M. 1994. Fossils of the Flaming Cliffs. Scientific American 271: 60-69.

Novacek, M.J., Rougier, G. W., Wible, J. R., McKenna, M. C., Dashzeveg, D., and Horovitz, I. 1997. Epipubic bones in eutherian mammals from the Late Cretaceous of Mongolia. Nature 389: 483-486.

Pol, D., and Norell, M. A. 2004. A new gobiosuchid crocodyliform taxon from the Cretaceous of Mongolia. American Museum Novitates 3458: 1-31

Presley, R. 1981. Alisphenoid equivalents in placentals, marsupials, monotremes and fossils. Nature 294(5842): 668-670.

Presley, R., and Steel, F. L. D. 1976. On the homology of the alisphenoid. Journal of Anatomy, London 121: 441-459.

Prothero, D. R., and Swisher III, C. C. 1992. Magnetostratigraphy and geochronology of the terrestrial eocene-oligocene transition in North America. In D. R. Prothero and W. A. Berggren (eds.). Eocene-Oligocene Climatic and Biotic Evolution: 46-73. Princeton: Princeton Univ. Press.

Rougier, G.W. 1993. Vincelestes neuquenianus Bonaparte (Mammalia, Theria) un primitivo mamífero del Cretácico Inferior de la Cuenca Neuquina. 720 pp. Ph.D. dissertation, Universidad Nacional de Buenos Aires, Buenos Aires.

Rougier, G. W. and Novacek, M. J. 1997. Nasal and endocranial morphology of Late Cretaceous multituberculates from Ukhaa Tolgod, Mongolia. Journal of Vertebrate Paleontology 17(3): 72A.

Rougier, G. W., Wible, J. R., and Novacek, M. J.1998. Implications of Deltatheridium specimens for early marsupial history. Nature 396: 459463.

Rougier, G. W., Novacek, M. J., and Dashzeveg, D. 1997. A new multituberculate from the Late Cretaceous locality Ukhaa Tolgod, Mongolia. Considerations on multituberculate interrelationships. American Museum Novitates 3191: $1-26$. 
Rougier, G. W., Wible, J. R., and Hopson, J. A. 1992. Reconstruction of the cranial vessels in the Early Cretaceous mammal Vincelestes neuquenianus: implications for the evolution of the mammalian cranial vascular system. Journal of Vertebrate Paleontology 12: 188-216.

Rougier, G. W., Wible, J. R., and Novacek, M. J.

1996a. Middle-ear ossicles of the multituberculate Kryptobaatar from the Mongolian Late Cretaceous: implications for mammaliamorph relationships and the evolution of the auditory apparatus. American Museum Novitates 3187: 1-43.

1996b. Multituberculate phylogeny. Nature. 379: 406.

1998. Implications of Deltatheridium specimens for early marsupial history. Nature 396: 450-463.

Rougier, G. W., Sheth, A. S., Carpenter, K., Appella-Guiscafre, L., and Davis, B. M. 2014. A new species of Docodon (Mammaliaformes: Docodonta) from the Upper Jurassic Morrison Formation and a reassessment of selected craniodental characters in basal mammaliaforms. Journal of Mammalian Evolution. DOI: 10.1007/s10914-014-9263-8

Rougier, G. W., and J. R. Wible. 2006. Major changes in the mammalian ear region and basicranium; pp. 269-311 In M. T. Carrano, T. J. Gaudin, R. W. Blob, and J. R. Wible (eds.). Amniote Paleobiology: Perspectives on the Evolution of Mammals, Birds, and Reptiles. University of Chicago Press, Chicago, Illinois.

Segall, W. 1970. Morphological parallelisms of bulla and auditory ossicles in some insectivores and marsupials. Fieldiana Zoology 51:169-205.

Shuvalov, V. F. 2000. The Cretaceous stratigraphy and palaeobiography in Mongolia. In M. J. Benton, M. A. Shishkin, E. N. Kurochkin, and D. M. Unwin (eds.). The Age of Dinosaurs in Russia and Mongolia, 256-278. Cambridge University Press, Cambridge.

Sidor, C. A. 2001. Simplification as a trend in synapsid cranial evolution. Evolution 55: 1419-1442.

Simmons, N. B.1993. Phylogeny of Multituberculata. Mammal Phylogeny: Placentals. New York, Springer Verlag Publishers: 146-164.

Simpson, G. G. 
1925. A Mesozoic mammal skull from Mongolia. American Museum Novitates 201: 12 pp.

1928a. A Catalogue of the Mesozoic Mammalia. London: The British Museum.

1928b. Affinities of the Mongolian Cretaceous insectivores. American Museum Novitates 330: 1-11.

1937. Skull structure of the multituberculata. Bulletin of the American Museum of Natural History 73: 727-763.

1938. Osteography of the ear region in monotremes. American Museum Novitates 978: 1-15.

Sisson, S. and J. D. Grossman. 1955. The Anatomy of the Domestic Animals. Saunders, Philadelphia. 972 pp.

Szalay, F. S. and Trofimov, B. A. 1996. The Mongolian Late Cretaceous Asiatherium, and the early phylogeny and paleobiogeography of Metatheria. Journal of Vertebrate Paleontology 16: 474-509.

Trofimov, B. A. and Szalay, F. S. 1994. New Cretaceous marsupial from Mongolia and the early radiation of Metatheria. Proceedings of the National Academy of Science 91: 12569-12573.

Wahlert, J. H.

1974. The cranial foramina of protogomorphous rodents; an anatomical and phylogenetic study. Bulletin of Museum of Comparative Zoology 146: 363-410.

1985. Cranial foramina of rodents. In Evolutionary relationships among rodents: a multidisciplinary approach. W. P. Luckett and J. L. Hartenberger, (eds.). Plenum Press. New York 311-332pp.

Wall, C. and Krause, D. W. 1992. A biomechanical analysis of the masticatory apparatus of Ptilodus (Multituberculata). Journal of Vertebrate Paleontology 12: 172-187.

Webster, D. B. 1966. Ear structure and function in modern mammals. American Zoologist 6: 451-466.

Webster, D. B., Webster, M. 1975. Auditory systems of Heteromyidae: functional morphology and evolution of the middle ear. Journal of Morphology 146: 343-376. 
Webster, D. B., Webster, M. 1984. The specialized auditory system of Kangaroo Rats. Contributions to Sesory Physiology 8: 161-196.

Wible, J. R. 1987. The eutherian stapedial artery: character analysis and implications for superordinal relationships. Zoological Journal of the Linnean Society 91: 107-135.

Wible, J.R., and Gaudin, T. J. 2004. The cranial osteology of the yellow armadillo Euphractus sexcinctus (Dasypodidae, Xenartrha, Placentalia). Annals of the Carnegie Museum 73: 117-196.

Wible, J. R., and Hopson, J. A. 1993. Basicranial evidence for early mammal phylogeny. In F. S. Szalay, M. J. Novacek, and M. C. McKenna (eds.). Mammal Phylogeny: Mesozoic Differentiation, Multituberculates, Monotremes, Early Therians, and Marsupials, 45-62. Springer-Verlag, New York.

Wible, J. R., and Rougier, G. W. 2000. Cranial anatomy of Kryptobaatar dashzevegi (Mammalia, Multituberculata), and its bearing on the evolution of mammalian characters. Bulletin of the American Museum of Natural History 247: 1-124.

Wible, J. R., Novacek, M. J., Rougier, G. W. 2004. New data on the skull and dentition in the Mongolian Late Cretaceous Eutharian mammal Zalambdalestes. Bulletin of the American Museum of Natural History. 281: 1-144.

Wilson, G., Alistair, E., Corfe, I., Smits, P., Fortelius, M., and Jernvall, J. 2012. Adaptive radiation of multituberculate mammals before the extinction of dinosaurs. Nature. 483: 457-460.

Zeller, U. 1989. Die entwicklung und morphologie des schadels von Ornithorhynchus anatinus (Mammalia: Prototheria: Monotremata). Abh. Senckenberg, Natuforsd. Ges. 545: 1-188.

Zheng, X., Shundong, B., Xiaoli, W., and Meng, J. 2013. A new arboreal haramyid shows the diversity of crown mammals in the Jurassic period. Nature. 500: 199-202.

Zhou, C-F., Wu, S., Martin, T., Luo, Z-X. 2013. A Jurassic mammaliaform and the earliest mammalian evolutionary adaptations. Nature. 500: 163-167. 


\section{APPENDICES}

Appendix A. Character list adapted from Kielan-Jaworowska and Hurum (1997), with the addition of character 44 for the post-palatine torus and the addition of Mangasbaatar udanii as a new taxon.

1. Enamel covering of lower incisor of uniform thickness (0), thicker on labial surface than on lingual surface (1), completely restricted to labial surface of tooth (2). Mangasbaatar (2)

2. p3 present (0), absent (1). Mangasbaatar (0)

3. p4 serration count 5 or less (0), 6-10 (1), more than 10 (2). Mangasbaatar (0) 4. p4 in lateral view rectangular (0), arcuate (1), trapezoidal (2), triangular (3). Mangasbaatar (2)

5. m1 cusp formula 4:3 (0), 4:4 (1), 5:4-5 (2), 7:4 or higher (3). Mangasbaatar (0) 6. Ratio of $\mathrm{p} 4: \mathrm{m} 1$ length less than $0.6(0), 0.6-1.7$ (1) above 1.7 (2).

Mangasbaatar (1)

7. m2 cusp formula 2-2 (0), more (1). Mangasbaatar (0)

8. 12 bicuspid (0), single-cusped (1). Mangasbaatar (?)

9. I3 located on margin of palate (0), slightly shifted from the labial margin (1), in about the middle of

the palatal part of the premaxilla (2). Mangasbaatar (2)

10. Upper premolars five (0), four (1), three (2) one (3). Mangasbaatar (2)

11. P3 double-rooted (0), single-rooted (1). Mangasbaatar (0)

12. P4 double-rooted (0), single-rooted (1). Mangasbaatar (0)

13. Length of upper premolar tooth row:molar tooth row more than $1.5(0), 1.54 .5$

(1), 0.5-0.1(2). Mangasbaatar (1)

14. P4 cusp formula 0-5:1-4:0-5 (0), 0-5:5-10:0-5 (1), 5-7:5-8:2-5 (2).

Mangasbaatar (1)

15. M1 cusp formula 4-5:4-5:0-5 (0), 5-7:5-8:2-5 (1), 5-11:7-10:611 (2).

Mangasbaatar $(0+1)$

16. M1 inner ridge length:length of M1 0.5 or less (0), more than 0.5 (1).

Mangasbaatar (0)

17. Width of P4:M1 ratio more than 0.9 (0), 0.9-0.6 (1), 0.6-0.45 (2), 0.45-0.2 (3). Mangasbaatar (2)

18. M2 cusp formula 1:2:2 (0). 1:2:3 (1), more (2). Mangasbaatar (0+1)

19. Ridge between the palate and the lateral walls of the premaxilla absent $(0)$, present (1). Mangasbaatar (1)

20. Shape of the snout in dorsal view: incurved in front of the zygomatic arches with anterior part 
directed posterolaterally (0), incurved with anterior part of zygomatic arches directed transversely

(1), trapezoidal, not incurved in front of zygomatic arches (2). Mangasbaatar (2)

21. Number of pairs of vascular foramina on nasal: 1 (0), 2 (1), more (2).

Mangasbaatar (?)

22. Infraorbital foramen positioned dorsal to P1 (0), dorsal to P2 (1), dorsal to P3 or P3 (2). Mangasbaatar (1)

23. Base of zygomatic arch as marked by posterior edge directly dorsal to P4 (0), dorsal or posterior to

P4/M1 embrasure (1). Mangasbaatar (0)

24. Postorbital process short (0), long (1). Mangasbaatar (1)

25 . Snout length $49 \%$ or less of total skull length (0), $50 \%$ or more of skull length

(1). Mangasbaatar (0)

26. Frontals pointed anteriorly and not deeply inserted between the nasals (0),

pointed anteriorly and

deeply inserted between the nasals (1), with subtransversal anterior margins (2).

Mangasbaatar (1)

27. Frontal-parietal suture V-shaped (0), U-shaped (1). Mangasbaatar (1)

28. Contacts between nasal and parietal absent (0), present (1). Mangasbaatar

(0)

29. Facial surface of lacrimal very small and arcuate (0), large, roughly

rectangular (1). Mangasbaatar (1)

30. Thickening in palatal process of premaxilla absent (0), present (1).

Mangasbaatar (1)

31. Incisive foramen situated within premaxilla (0), limited posteriorly by maxilla

(1). Mangasbaatar (1)

32. Palatal vacuities absent (0), single (1), double (2). Mangasbaatar (0)

33. Foramen ovale inferium placed medial to foramen masticatorium (0),

posterior to foramen masticatorium (1). Mangasbaatar (0)

34. Jugular fossa small and shallow (0), large and deep (1). Mangasbaatar (1)

35. Anterior part of the promontorium oval (0), irregular with incurvatures on both sides (1). Mangasbaatar (1)

36. Glenoid fossa (anterolateral to posteromedial) length:width ratio more than

1.7 (0), below 1.69 (1). Mangasbaatar (1)

37. Angle of coronoid process relative to tooth row steep, 45 degrees or $>45$

degrees (0), low < 45 degrees (1). Mangasbaatar (0)

38. Coronoid process parallel to the rest of the outer wall of the dentary $(0)$,

flared laterally (1). Mangasbaatar (0)

39. Posttemporal fossa large (0), reduced to a small foramen (1). Mangasbaatar

(0)

40. Angle between the lower margin of the dentary and the occlusal level of the molars between 11-20 degrees (0), above 20 degrees (1). Mangasbaatar (0) 41. Mandibular condyle opposite or below the level of the molars (0), above the level of the molars (1). Mangasbaatar (1)

42. Width of the snout:skull length ratio below $0.3(0), 0.3-0.39$ (1), above 0.4 (2).

Mangasbaatar (2) 
43. Skull width:skull length ratio 0.79 and below (0), above 0.8 (1). Mangasbaatar (1)

44: Post-palatine torus absent or very faint (0), developed laterally and with a venral projection from the palate, forming a distinctive bulge (1), strongly developed, forming a raised, ornate and sharply angled plate (2). Mangasbaatar (2)

Appendix B. Scored character matrix, adapted from Kielan-Jaworowska and Hurum (1997) for 42 characters across 17 taxa including Ptilodus, Stigymys, Taeniolabis, Eucosmodon, Lambdopsalis, Sloanbaatar, Buginbaatar, Kamptobaatar, Nemegbaatar, Chulsanbaatar, Kryptobaatar, Djadochtatherium, Catopsbaatar, Tombaatar and Mangasbaatar. "A" denotes multi-state $(0+1)$.

Ptilodus
Stigymys
Taeniolabis
Eucosmodon
Lambdopsalis
Sloanbaatar
Buginbaatar
Nessovbaatar
Bulganbaatar
Kamptobaatar
Nemegbaatar
Chulsanbaatar
Kryptobaatar
Djadochtatherium
Catopsbaatar
Tombaatar
Mangasbaatar

Ptilodus Stigymys Fucosmodon Lambdopsalis Sloanbaatar Nemegbaatar Chulsanbaatar yptobaatar Catopsbatar Mangasbaatar
$00212211010002210200120 ? 00000011000100000100$ 21213210211011102 ??0?00??????111????10?0???? 2103311103 ?120213201?2??020100?0????001 ?1110

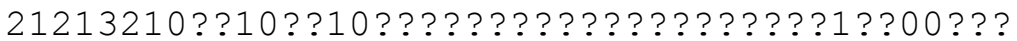
2103201113?120213201221?02010010?11?0?101110 $101101012100110021100100011011020 ? 0001011010$ 0101301 ?? ? 0 ? 12132 ? ? ? ? ? ? ? ? ? ? ? ? 0 ? 0 ? ? ?

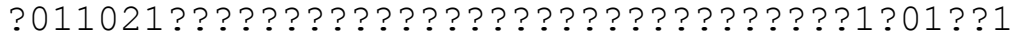
?? ??? 1210011011010 ?10 ??? ?11 ?1 ?????? ??? 101101 ? $1210011012110200001101110 ? 10011001101$ 20112111210011111210210001101111010010000101 20110101210011002010100001101110110110000001 2011011121001100211211001110111011111000021 ? 20 ?101?121???0????12?1011110111 ?????00001 ??? $20021101220011112212121111101100 ? 11100011211$ 2??????1220011002112110??1?01110???????????2 2002010?220011A02A12?10101101110011100001212 
CURRICULUM VITAE

Amir Subhash Sheth

\section{ADDRESS:}

1708 Shady Lane,

Louisville, Ky, 40205

(502) 338-9806

E-mail: amirssheth@gmail.com

DOB:

June 7, 1988

\section{EDUCATION:}

Centre College, Danville, Ky

B.A. in Music with a minor in Philosophy, June 2010

University of Louisville, Louisville, Ky

M.S. in Anatomical Sciences and Neurobiology (currently completing $2^{\text {nd }}$ year)

\section{Kentucky Country Day School}

High school diploma, June 2006

\section{AWARDS:}

Recipient of Connect-The-Docs scholarship - Physassist Scribes, inc. May, 2013

\section{PUBLICATIONS:}

Rougier, G. W. and Sheth, A. S. 2013. Paleontology and paleontologists: A personal account of Mesozoic mammals and the people who study them. Book review of: In Pursuit of Early Mammals. Zofia Kielan-Jaworowska. 
Indiana University Press, Bloomington, IN, USA. 253 pp, ISBN 978-0-25300817-6.

Rougier, G. W., Sheth, A. S., Carpenter, K., Appella-Guiscafre, L., and Davis, B. M. 2014. A new species of Docodon (Mammaliaformes: Docodonta) from the Upper Jurassic Morrison Formation and a reassessment of selected craniodental characters in basal mammaliaforms. Journal of Mammalian Evolution. DOI: 10.1007/s10914-014-9263-8

\section{TEACHING EXPERIENCE:}

\section{Centre College}

Tutor in music and assistant to the professor

\section{University of Louisville}

Athletic tutor (Mathematics, Chemistry, Biology, Logic), 2010-2012

Completed Graduate Teaching Assistant Academy 2013-2014

WORK:

Physassist Scribes, inc.

Scribe, Norton Audubon hospital, Emergency Department, June 2012 - June 2013

\section{OTHER:}

Jazz \& Bluegrass musician and instructor with expertise in 5 instruments: violin, mandolin, guitar, bass guitar and percussion.

Capable of conversation in Spanish

Runner up in Kentucky State Competition, Track and Field, 400m dash 